\title{
Noncommutative Geometry in M-Theory and Conformal Field Theory
}

\author{
Bogdan Morariu \\ Ph.D. Thesis \\ Department of Physics \\ University of California, Berkeley \\ and \\ Physics Division \\ Ernest Orlando Lawrence Berkeley National Laboratory \\ University of California \\ Berkeley, CA 94720
}

May 1999

This work was supported in part by the Director, Office of Science, Office of High Energy and Nuclear Physics, Division of High Energy Physics, of the U.S. Department of Energy under Contract No. DE-AC03-76SF00098, and the National Science Foundation under Grant No. PHY-95-14797. 


\section{DISCLAIMER}

This report was prepared as an account of work sponsored by an agency of the United States Government. Neither the United States Government nor any agency thereof, nor any of their employees, make any warranty, express or implied, or assumes any legal liability or responsibility for the accuracy, completeness, or usefulness of any information, apparatus, product, or process disclosed, or represents that its use would not infringe privately owned rights. Reference herein to any specific commercial product, process, or service by trade name, trademark, manufacturer, or otherwise does not necessarily constitute or imply its endorsement, recommendation, or favoring by the United States Government or any agency thereof. The views and opinions of authors expressed herein do not necessarily state or reflect those of the United States Government or any agency thereof. 


\section{DISCLAIMER}

Portions of this document may be illegible in electronic image products. Images are produced from the best available original document. 


\section{NONCOMMUTATIVE GEOMETRY IN M-THEORY AND CONFORMAL FIELD THEORY}

by

\section{Bogdan Morariu}

GRAD. (Bucharest University) 1986

A dissertation submitted in partial satisfaction of the

requirements for the degree of

Doctor of Philosophy in Physics

in the

GRADUATE DIVISION

of the

UNIVERSITY of CALIFORNIA, BERKELEY

Committee in charge:

Professor Bruno Zumino, Chair

Professor Korkut Bardakci

Professor Nicolai Y. Reshetikhin 


\title{
Noncommutative Geometry in M-Theory and Conformal Field Theory
}

\author{
Copyright $\odot 1999$
}

by

Bogdan Morariu

The U.S. Department of Energy has the right to use this document for any purpose whatsoever including the right to reproduce all or any part thereof. 


\author{
Abstract \\ CONFORMAL FIELD THEORY \\ by

\section{Bogdan Morariu} \\ Doctor of Philosophy in Physics \\ University of California, Berkeley \\ Professor Bruno Zumino, Chair
}

NONCOMMUTATIVE GEOMETRY IN M-THEORY AND

In the first part of the thesis I will investigate in the Matrix theory framework, the subgroup of dualities of the Discrete Light Cone Quantization of M-theory compactified on tori, which corresponds to T-duality in the auxiliary Type II string theory. After a review of matrix theory compactification leading to noncommutative supersymmetric Yang-Mills gauge theory, I will present solutions for the fundamental and adjoint sections on a two-dimensional twisted quantum torus and generalize to three-dimensional twisted quantum tori. After showing how M-theory T-duality is realized in supersymmetric Yang-Mills gauge theories on dual noncommutative tori I will relate this to the mathematical concept of Morita equivalence of $C^{*}$-algebras. As a furthure generalization, I consider ar- 
bitrary Ramond-Ramond backgrounds. I will also discuss the spectrum of the toroidally compactified Matrix theory corresponding to quantized electric fluxes on two and three tori.

In the second part of the thesis I will present an application to conformal field theory involving quantum groups, another important example of a noncommutative space. First, I will give an introduction to Poisson-Lie groups and arrive at quantum groups using the Feynman path integral. I will quantize the symplectic leaves of the Poisson-Lie group $S U(2)^{*}$. In this way we obtain the unitary representations of $\mathcal{U}_{q}(s u(2))$. I discuss the $*$-structure of $S U(2)^{*}$ and give a detailed description of its leaves using various parametrizations. Then, I will introduce a new reality structure on the Heisenberg double of $\operatorname{Fun}_{q}(S L(N, \mathrm{C}))$ for $q$ phase, which can be interpreted as the quantum phase space of a particle on the $q$-deformed mass-hyperboloid. I also present evidence that the above real form describes zero modes of certain non-compact WZNW-models. 
To Kitty 


\section{Acknowledgements}

First, it is my great pleasure to thank my advisor Professor Bruno Zumino for his constant guidance, encouragement, his insights into many fascinating aspects of theoretical physics, his sense of humor, and for creating a research environment at once stimulating and free. I also thank him for his invaluable contribution to our collaborations. I feel very privileged to have been his student.

I must also thank my fellow graduate students Harold Steinacker, Paolo Aschieri, Chong-Sun Chu, Pei-Ming Ho, Michael Graesser, and especially my collaborator Dan Brace for their friendship and for making Berkeley such an enjoyable place. Much of the physics that I really understand was crystallized during the long informal discussions we had.

I would like to thank Professors Korkut Bardakci, Hirosi Ooguri, Martin Halpern, and Nicolai Reshetikhin who tought me a great deal, and whose doors were always open for my questions.

My thanks also go to the extremely kind and helpful staff on campus and at the lab, Laura Scott, Anne Takizawa and Donna Sakima, Luanne Neumann, Barbara Gordon and Mary Kihanya.

I am indebted to my parents for supporting me in my early decision to study physics and for patiently awaiting my graduation day.

Most of all, this thesis would not have been possible without Kitty's understanding and generosity. I would like to thank her for sharing with me the last few years, for tolerating my absent-mindedness while absorbed in some physics project, and for sometimes even allowing me to explain to her some exciting new physics discovery that I barely understood. 
This work was supported in part by the Director, Office of Energy Research, Office of High Energy and Nuclear Physics, Division of High Energy Physics of the U.S. Department of Energy under Contract DE-AC03-76SF00098 and in part by the National Science Foundation under grant PHY-95-14797. 


\section{Contents}

1 Introduction and Outline $\quad 1$

1.1 Toroidal Compactification of the DLCQ of M-theory . . . . . . 12

1.2 Path Integral Quantization of Poisson-Lie Groups and Reality Structures on Quantum Cotangent Bundles . . . . . . . . . 17

2 Super Yang-Mills on the Noncommutative Torus 22

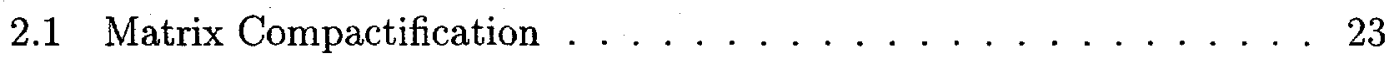

2.2 Twisted Quantum Bundles on $T^{2} \ldots \ldots$. . . . . . . 30

2.3 Twisted Quantum Bundles on Tori ............. . . 39

2.4 Adjoint Sections on Twisted Bundles . . . . . . . . . . . . 42

2.5 Two and Three Dimensional Solutions . . . . . . . . . . . 48

2.6 Noncommutative Super Yang-Mills Action . . . . . . . . . . 54

3 Dualities of the Matrix Model from T-Duality of the Type II

$\begin{array}{ll}\text { String } & 57\end{array}$

$3.1 S O(3,3 \mid \mathbf{Z})$ Duality of Super Yang-Mills . . . . . . . . . . . 57

3.2 Target Space Duality . . . . . . . . . . . . . 62

3.3 Projective Modules and Morita Equivalence . . . . . . . . 66

3.4 Gauge Transformations . . . . . . . . . . . . . . 72

4 Adding Ramond-Ramond Backgrounds $\quad 75$ 
4.1 Duality of Seven Dimensional Supergravity . . . . . . . . . . 76

4.2 T-duality of the Chern-Simon Type Terms . . . . . . . . . . 80

5 T-duality of the BPS Spectrum $\quad 88$

5.1 The $U(1)$ BPS Spectrum . . . . . . . . . . . . . 90

5.2 Dual $U(n)$ BPS Spectrum . . . . . . . . . . . . 92

6 Path Integral Quantization of the Symplectic Leaves of the $S U(2)^{*}$

$\begin{array}{ll}\text { Poisson-Lie Group } & 98\end{array}$

6.1 Dual Pairs of Poisson-Lie Groups . . . . . . . . . . . 99

6.2 Symplectic Leaves . . . . . . . . . . . . . . . . . 107

6.3 Path Integral Quantization . . . . . . . . . . . 111

6.4 Matrix Elements and the Quantum Algebra . . . . . . . . 116

6.5 Remarks . . . . . . . . . . . . . . . 120

7 Quantum Algebra of the Particle Moving on the $q$-Deformed Mass-Hyperboloid 123

7.1. Review of the Algebra on $\mathrm{T}^{*} G_{q} \ldots \ldots \ldots . \ldots . \ldots 23$

7.2 Real Form for the $q$-Deformed Symmetric Top . . . . . . . . . 129

7.3 Real Form for the $q$-Deformed Hyperboloid . . . . . . . . . . . 130

7.4 Quantum Mechanics on the $q$-Deformed Hyperboloid . . . . . . 132

7.5 Lattice regularized WZNW-model . . . . . . . . . . 135

A Chiral Spinor Representations of $S O(d, d \mid \mathrm{Z}) \quad 137$ 


\section{Chapter 1}

\section{Introduction and Outline}

String theory is the most promising candidate for a unified theory of gravity with the other interactions already present in the standard model of particle physics. Five consistent string theories are known to exist in ten dimensions. One of them is an open unoriented superstring theory called Type I. There are two closed superstring theories called Type IIA and Type IIB, depending on whether the GSO projection on left and right movers leads to a nonchiral or chiral spacetime theory. Finally there are two heterotic string theories, one with gauge group $S O(32)$ and one with gauge group $E_{8} \times E_{8}$. Lower dimensional string theories can then be obtained by compactification on a small manifold. Due to the large number or resulting theories it seemed that one of the initial promising features of string theory, its uniqueness, was lost. Furthermore, some of these theories do not have a unique classical vacuum and perturbative corrections do not remove this degeneracy.

Some hope existed that nonperturbative corrections would select a specific vacuum, perhaps the one describing our world. However, only a perturbative formulation of these string theories is available, and it seemed until recently that no real progress could be made without a full nonperturbative formulation of 
string theory. Nevertheless, access to nonperturbative aspects of string theories was possible though an analysis of their low energy formulation as supergravity theories.

A large number of nonperturbative supergravity solutions were found and used to access the strong coupling regime of string theory. This is possible in theories with extended supersymmetry by using the so called BPS states, which are states in small representations of the supersymmetry algebra. Since the dimension of these representations is an integer which cannot vary continuously, the states in these short multiplets cannot leave the representation as one changes continuous parameters, such as the coupling constant. Most importantly, for BPS states the supersymmetry algebra requires certain relations between the masses and charges be satisfied, and this allows one to know the masses of these states even at strong coupling.

An important discovery due to Polchinski [58] was that the nonperturbative solutions of the low energy supergravity theories which carry Ramond-Ramond charges could be understood directly in the world sheet conformal field theory describing the string dynamics as hypersurfaces in space-time where strings can end, named Dirichlet branes or D-branes for short.

The surprise came in 1995 when it was realized $[77,85]$ that most of the known string theories at strong coupling were not new, strange and hard to understand theories but in fact were described by other already known string theories. This relation between different string theories is known as string duality. Evidence for 
these dualities can be obtained by trying to identify in one theory nonperturbative massive BPS states which when followed to strong coupling, become the light degrees of freedom of the theory, and can be identified as the fundamental degrees of freedom of the dual theory. All the known string theories were unified into a web of theories with each theory at strong coupling or small volume equivalent to another theory at weak coupling or large volume. The relation between large and small volume theories had been known since the late nineteen-eighthes as target space duality or T-duality. For example Type IIA and Type IIB are T-dual to each other after toroidal compactification. A more complicated version of target space duality occurs for compactification on Calabi-Yau spaces and is known as mirror symmetry. A further unification discovered by Strominger [75] involves theories compactified on Calabi-Yau manifolds of different topology. String theory can move continuously from one such theory to another using the the conifold transition. This is a mechanism involving massless black hole condensation.

However not all the corners of this web were described by known string theories. In one particular corner describing the strong coupling dual of Type IIA string theory was an eleven dimensional theory whose low energy description was given by eleven dimensional supergravity. This is the theory with the largest allowed number of supercharges, but it is nonrenormalizable. It was conjectured that there exists a consistent eleven dimensional theory, called M-theory, such that its low energy is eleven dimensional supergravity. Sometimes the name M-theory is used to describe the whole web and sometimes only the strong coupling dual of the 
Type IIA string.

The main thrust of the first part of this thesis is the study of the toroidal compactification of the discrete light cone quantization of M-theory in the presence of arbitrary moduli. The main mathematical ingredient in this study to which I will turn next is noncommutative geometry. The common thread throughout this thesis is the appearance of noncommutative spaces playing various roles in examples such as M-theory or conformal field theory. Yet it is not the familiar and venerable role of the algebra of observables of a quantum dynamical system.

Noncommutative spaces have existed in physics ever since the creation of quantum mechanics, where they first appeared as the quantum correspondent of the phase space of a classical system. More recently quantum spaces such as quantum groups have played a role in describing generalized symmetries. In another important context, quantum spaces have appeared as quantizations not of the dynamical variables but of some auxiliary space such as the world volume coordinates of a field theory. Gauge theory on the noncommutative torus is such an example which has recently found a prominent role in the compactification of M-theory. Quantization of an auxiliary space has also been used as a regularization method. The low energy action of D-brane probes on orbifolds with torsion is another recent example where noncommutative geometry plays a role. In these theories the auxiliary quantum spaces do not have a classical limit, which can only exist if we have a continuous parameter such as $\hbar$ which can be tuned to zero.

Next I will present a brief introduction to the main ideas of noncommutative 
geometry. A classic mathematical result of Gel'fand states that compact topological spaces are in one-to-one correspondence with commutative $C^{*}$-algebras. In one direction, to a topological space $X$, one can associate the algebra of continuous functions $C(X)$. Conversely and rather nontrivially, the spectrum of a commutative $C^{*}$-algebra is equivalent to a compact topological space. This important result allows for a dual description of topological spaces and brings powerful algebraic methods into the realm of topology. On the other hand, if one drops the commutativity requirement, a $C^{*}$-algebra $\mathcal{A}$ describes what is called by correspondence a quantum space.

To illustrate, consider the algebra of functions on a two-torus $C\left(T^{2}\right)$. An arbitrary element $f$ of this algebra has the Fourier expansion

$$
f=\sum_{k, l \in \mathbf{Z}} f_{k, l} U_{1}^{k} U_{2}^{l}
$$

where $U_{i}=e^{\sigma_{i}}$ are the generators of this algebra. In general some restrictions are imposed on the $c$-number coefficients $f_{k, l}$ (such as they form a square-summable or rapidly decreasing sequence). Very importantly (1.1) is a global statement. In the opposite direction, if we know that all the algebra elements have the form (1.1) we immediately recognize that they can be identified with functions on a two-torus. Thus one can read the topological compact space from the commutative algebra. Since $\sigma_{i}$ are local coordinates on the torus the generators $U_{i}$ commute. Instead we can consider the algebra whose elements have the form (1.1) but with $U_{i}$ 's 
satisfying

$$
U_{1} U_{2}=e^{2 \pi i \theta} U_{2} U_{1}
$$

where $\theta$ is a real number. This algebra is known as the algebra of 'functions' on the noncommutative torus

As $\theta$ goes to zero we obtain the algebra of functions on the commutative torus. However we can endow this algebra with additional structure, making it into a Poisson algebra. For two elements $f_{1}$ and $f_{2}$ of $C\left(T^{2}\right)$ we define the following Poisson bracket

$$
\{f, h\}=\lim _{\theta \rightarrow 0} \frac{1}{-2 \pi i \theta}[\tilde{f}, \tilde{h}]=\varepsilon_{i j} \frac{\partial f}{\partial \sigma_{i}} \frac{\partial h}{\partial \sigma_{j}} .
$$

Above $\tilde{f}$ and $\tilde{h}$ are elements of the algebra of the quantum torus with deformation parameter $\theta$ which reduce to $f$ and $h$ in the classical limit.

This process can sometimes be reversed. Given a Poisson algebra we can try to construct a quantum algebra such that it reduces to the Poisson algebra in the classical limit. Using the same strategy as discussed above for the torus one can describe other spaces in commutative algebraic terms and then remove the commutativity requirement. One of the most interesting examples is given by Poisson-Lie groups and their noncommutative generalization, quantum groups.

While the quantum correspondent of a topological space is a $C^{*}$-algebra, other spaces of classical geometry with additional structure can be described in different terms. For example vector bundles can be equivalently described if we can characterize all their sections. As will be explained in some detail later, the set of sections of a vector bundle has the structure of a projective module over the alge- 
bra of functions on the base manifold. Then projective modules over a quantum algebra are the generalization of classical vector bundles.

In the last two chapters, I will consider some applications of Poisson-Lie groups and their noncommutative correspondents, quantum groups. Quantum groups were introduced as a tool for studying deformations of lower dimensional quantum mechanical systems. It was also discovered that Poisson-Lie groups play a role in the study of their classical counterpart. What emerged was the notion of Poisson symmetry which I will briefly describe next.

Let $\mathcal{M}$ be the phase space of a mechanical system with Hamiltonian $H$ and $G$ a Lie group acting on this phase space. The point $x \in \mathcal{M}$ is mapped into $g(x)$ under the action of the group element $g$. Then for $f \in C(\mathcal{M})$ some arbitrary dynamical variable we can define a new function $\tilde{f} \in C(G \times \mathcal{M})$ by ${ }^{1}$

$$
\tilde{f}(g, x)=f(g(x)) .
$$

The evolution of the system is described by the Poisson equation

$$
\dot{f}=\{f, H\}_{\mathcal{M}} .
$$

The action of $G$ is a symmetry of the dynamics if it commutes with the evolution generated by the Hamiltonian $H$. Let $v_{H}$ be the Hamiltonian vector field associated to $H$, i.e. its action on functions is given by

$$
v_{H} f=\{f, H\} .
$$

\footnotetext{
${ }^{1}$ As I will discuss later, the translation of an action on a manifold into its dual correspondent on functions over that manifold is called a coaction.
} 
One can lift the action of $G$ from the phase space $\mathcal{M}$ to its tangent bundle $T(\mathcal{M})$

$$
\widetilde{v} f=v \widetilde{f}
$$

Here $\tilde{v}$ is the resulting vector field under the action by a yet unspecified group element. Then we have a symmetry if the Hamiltonian vector field is left invariant by this action

$$
\widetilde{v_{H}} f=\widetilde{v_{H} f}
$$

The standard textbook symmetry is given by a group action such that both the Hamiltonian and the Poisson bracket are left invariant

$$
\begin{gathered}
\tilde{H}(g, x)=H(x), \\
\left\{\widetilde{f}_{1}, \widetilde{f}_{2}\right\}_{\mathcal{M}}=\left\{\widetilde{f_{1}, f_{2}}\right\}_{\mathcal{M}} .
\end{gathered}
$$

Then the proof of symmetry (1.4) immediately follows

$$
\widetilde{v_{H}} f=v_{H} \tilde{f}=\{\widetilde{f}, H\}_{\mathcal{M}}=\{\widetilde{f}, \widetilde{H}\}_{\mathcal{M}}=\{\widetilde{f, H}\}_{\mathcal{M}}=\widetilde{v_{H} f}
$$

The first equality is just the definition of the lift, the third is implied by the invariance of the Hamiltonian (1.5) and the fourth by the invariance of the Poisson bracket (1.6).

However a more general notion of symmetry emerges if one also endows the group $G$ with a Poisson bracket $\{,\}_{G}$. If the Poisson structure is compatible with the group operations the group is called a Poisson-Lie group. A detailed description of this will be given in Chapter 6 . With these ingredients one can also 
define a Poisson bracket on $G \times \mathcal{M}$ by the requiring that it satisfies

$$
\begin{aligned}
\left\{f_{1}, f_{2}\right\}_{G \times \mathcal{M}} & =\left\{f_{1}, f_{2}\right\}_{\mathcal{M}}, f_{1}, f_{2} \in C(\mathcal{M}) \\
\left\{f_{1}, f_{2}\right\}_{G \times \mathcal{M}} & =\left\{f_{1}, f_{2}\right\}_{G}, f_{1}, f_{2} \in C(G) \\
\left\{f_{1}, f_{2}\right\}_{G \times \mathcal{M}} & =0, f_{1} \in C(\mathcal{M}), f_{2} \in C(G)
\end{aligned}
$$

This is the natural Poisson bracket on a cross product space. It reduces to the bracket on each factor for functions independent of the coordinates of the other factor, and vanishes between mixed coordinates.

One can generalize the invariance (1.6) of the bracket $\{,\}_{\mathcal{M}}$ under the group action. The action of the group is called a Poisson action if it satisfies

$$
\left\{\widetilde{f}_{1}, \tilde{f}_{2}\right\}_{G \times \mathcal{M}}=\left\{\widetilde{f_{1}, f_{2}}\right\}_{\mathcal{M}}
$$

for any $f_{1}, f_{2} \in C(\mathcal{M})$. Note that the condition (1.8) to have a Poisson action involves the bracket on the group and reduces to the standard invariant action (1.6) if we take the trivial bracket on the group $\{,\}_{G}=0$.

If one has Poisson invariance then the following sequence

$$
\widetilde{v_{H}} f=v_{H} \tilde{f}=\{\widetilde{f}, H\}_{\mathcal{M}}=\{\tilde{f}, H\}_{G \times \mathcal{M}}=\{\tilde{f}, \widetilde{H}\}_{G \times \mathcal{M}}=\{\widetilde{f, H}\}_{\mathcal{M}}=\widetilde{v_{H} f}
$$

shows that we still have a symmetry. It is usually called Poisson symmetry. In the third equality I used the fact that $H$ is $g$ independent and (1.7), in the fourth equality the invariance of the Hamiltonian under the group action and in the fifth the fact that $G$ has a Poisson action on $\mathcal{M}$. 
This has been extensively used in the study of one plus one dimensional integrable models. It has also emerged as a hidden symmetry of the Wess-ZuminoNovikov-Witten (WZNW) model. Upon quantization of these dynamical systems one has to simultaneously quantize the Poisson structure on the group and the resulting object is called a quantum group. One can then continue to use the power of symmetry for examlpe to organize the physical states in representations of the quantum group.

Quantum groups might also play a role in the $A d S / C F T$ correspondence. Set forward by Maldacena, this states that string theory on Anti-de-Sitter multiplied by a compact manifold is equivalent to a conformal field theory on the boundary of $A d S$. In the case of $A d S_{3} \times S^{3}$ the unitarity bound on the charges of chiral primary fields of any of the $N=2$ superconformal subalgebras of the boundary theory is translated under the correspondence into a stringy exclusion principle of the quantum theory on $A d S_{3} \times S^{3}$. A proposed explanation involves nonperturbative contributions which effectively transform $A d S_{3} \times S^{3}$ in their $q$-deformed counterparts where $q$ is a root of unity and is determined by the central charge of the CFT theory on the boundary. If the Kaluza Klein modes for a scalar field are obtained by solving the wave equation on the $q$-deformed quantum space, ${ }^{2}$ the number of multi-particle states is finite and agrees with the number of chiral primary operators of the CFT on the boundary.

\footnotetext{
${ }^{2}$ Both $A d S_{3}$ and $S^{3}$ are group manifolds and one uses standard quantum groups to describe their quantization.
} 
I would like to mention an important application of the Poisson bracket (1.3). It was used in [83] by de Wit, Hoppe and Nicolai to write the light-cone Hamiltonian of a toroidal membrane [83] in a form that closely resembles the Hamiltonian of the Matrix model $[20,32,8]$. The world volume theory of the membrane is a gauge theory with the group of area preserving diffeomorphisms as the gauge group. One can use the Poisson bracket (1.3) to express the variation of functions on the world volume of the membrane under diffeomorphisms generated by infinitesimal Hamiltonian vector fields i.e. whose components have the form $v_{i}=\varepsilon_{i j} \partial h / \partial \sigma_{j}$. Then

$$
\delta f=v_{i} \frac{\partial f}{\partial \sigma_{i}}=\{f, h\} .
$$

If $\theta$ is rational one can find finite dimensional representations of the algebra (1.2). Then finite dimensional matrices can be expanded as

$$
X=\sum_{k, l \in \mathbf{Z}_{n}} X_{k l} U_{1}^{k} U_{2}^{l}
$$

where now the sum is over a finite range. It was shown in [83] that one can regularize the membrane theory by quantizing the world volume Poisson bracket. This is done by expanding all the fields in the maximally supersymmetric Matrix model action using (1.9) and, in the large $n$ limit, identifying the coefficients $X_{k l}$ with the Fourier modes of the supercoordinates of a toroidal membrane. This was the first appearance of the Matrix model in relation to the eleven dimensional theory known today as M-theory.

Banks, Fishler, Shenker and Susskind (BFSS) in [9] turned the relation between 
the membrane and Matrix model upside-down. Instead of thinking of the Matrix model as an auxiliary theory used for regularization purposes, they conjectured that the large $n$ Matrix model is M-theory. Membranes are then obtained as low energy excitations, but the Matrix model also describes a whole zoo of other states such as M5-branes, or after compactification to lower dimensions, strings and D-branes.

\subsection{Toroidal Compactification of the DLCQ of M-theory}

A further refinement of the BFSS conjecture was given by Susskind [76] who proposed that each momentum sector of the discrete light cone quantization (DLCQ) of M-theory is described by a maximally supersymmetric Matrix model with the momentum identified with the rank of the gauge group. The conjecture was further clarified by Sen and Seiberg $[73,69]$. They used an infinite boost and a compensating rescaling to show that the DLCQ Hamiltonian of the original M-theory where the light-cone variable is identified with period $L$ is given by the Hamiltonian of an auxiliary M-theory compactified on a vanishingly small space-like circle of radius $R$. This is then equivalent to a weakly coupled Type IIA string theory, which will be referred to, following Sen [74], as the auxiliary Type II string theory. At the same time, the original light-cone momentum is mapped into Ramond-Ramond D0 brane charge. The string coupling and string mass scale 
are given by the $R \rightarrow 0$ limit of

$$
g_{S}=M_{P}^{3 / 2}(L R)^{3 / 4}, m_{S}=M_{P}^{3 / 2} L^{3 / 4} R^{-1 / 4}
$$

where $M_{P}$ is the eleven dimensional Planck mass.

In this limit as proposed by Witten [87], and discussed extensively in [26], the excited string states decouple and the dynamics of $n$ D0 branes is determined by the maximally supersymmetric Matrix model $[20,32,8]$.

Toroidal compactification of M-theory can be obtained by considering Matrix theory on the covering space of the torus and imposing a periodicity constraint on the dynamical variables $[9,78,33]$. The constrained system is formally equivalent to a $U(n)$ super Yang-Mills (SYM) gauge theory on a dual torus. On the other hand, upon compactification on a $d$-dimensional torus $T^{d}$, M-theory has additional moduli from the three form of eleven dimensional supergravity. Connes, Douglas and Schwarz [24], conjectured that these moduli correspond to the deformation parameters $\Theta_{i j}$ of a noncommutative super Yang-Mills (NCSYM) gauge theory. Further studies of this subject followed in $[27,37,38,17,21,46,67,7,39,13,68$, $49,52,6]$.

In [24], where compactification on a two-torus was considered in some detail, it was suggested that the $S L(2, \mathbf{Z})$ noncommutative duality group of the NCSYM gauge theory $[60,63,64,65,66]$ corresponds to the T-duality in the DLCQ direction and one of the space-like compact directions of M-theory. However later Rieffel and Schwarz [67] showed that NCSYM gauge theories on higher dimensional 
tori have an $S O(d, d \mid \mathbf{Z})$ duality, and conjectured that this is the realization, in the NCSYM theory, of the auxiliary Type II string theory T-duality.

In general, as will be extensively discussed in the first part of this thesis, two NCSYM theories are dual to each other if there exists an element $\Lambda$ of the duality group $S O(d, d \mid \mathrm{Z})$ with the block decomposition ${ }^{3}$

$$
\Lambda=\left(\begin{array}{ll}
\mathcal{A} & \mathcal{B} \\
\mathcal{C} & \mathcal{D}
\end{array}\right)
$$

such that their defining parameters are related as follows

$$
\begin{aligned}
\bar{\Theta} & =(\mathcal{A} \Theta+\mathcal{B})(\mathcal{C} \Theta+\mathcal{D})^{-1} \\
\bar{G}^{i j} & =(\mathcal{C} \Theta+\mathcal{D})_{k}^{i}(\mathcal{C} \Theta+\mathcal{D})_{l}^{j} G^{k l}, \\
\bar{g}_{S Y M}^{2} & =\sqrt{|\operatorname{det}(\mathcal{C} \Theta+\mathcal{D})|} g_{S Y M}^{2}, \\
\bar{\eta} & =S(\Lambda) \eta \\
\bar{\chi} & =S(\Lambda) \chi .
\end{aligned}
$$

Here $S(\Lambda)$ denotes the Weyl spinor representation of $\Lambda$. The deformation parameter of the noncommutative torus $\Theta$ is a $d$-dimensional antisymmetric matrix, $G^{i j}$ is the metric defining the torus of the NCSYM, and $g_{S Y M}$ is the gauge coupling constant. The integral chiral spinor $\eta$ contains the Chern numbers of the bundle. For example, for compactification on a three torus, $\eta$ contains the rank of the group and the magnetic fluxes, and for a four torus it also includes the instanton number. The chiral spinor $\chi$ in (1.15) determines the parameters of

\footnotetext{
${ }^{3}$ The $S O(d, d \mid \mathbf{Z})$ subgroup of the T-duality group $O(d, d \mid \mathbf{Z})$ is the subgroup that does not exchange Type IIA and IIB string theories.
} 
the Chern-Simon type terms which can be added to the NCSYM action. In the auxiliary Type IIA string theory $\chi$ is closely related to the R-R moduli.

The relation (1.11) was first written in [67]. Equation (1.14) appeared in [68] where $S(\Lambda)$ was identified as a canonical transformation and was independently found in [14] and identified as a chiral spinor transformation. Equation (1.12) was implicit in [68] and was first written in [14] where equation (1.13) was also derived. Finally, deriving (1.15) was the main thrust [16].

The first part of this thesis is an investigation of this duality conjecture and some of its consequences. In Chapter 2 an extension of the method used in $[39,52]$ will be employed to construct twisted bundles to the two and three-tori. Then I will explain in some detail how to solve the boundary conditions for sections in the fundamental and adjoint quantum bundle. Using the special form of the transition functions in the given gauge, I will find different equivalent forms of the general solution for fundamental sections.

In Chapter 3, I will show explicitly how to construct an action of the duality group $S O(d, d \mid \mathbf{Z})$ on NCSYM theories. Under these duality transformations the rank of the gauge group and the magnetic flux numbers transform together in a Weyl spinor representation, and the deformation parameters transform by fractional transformations. I will also obtain the transformation properties, under the duality group, of the gauge coupling and the metric. One can then directly compare these relations with the string theory T-duality predictions. I will then discuss the more abstract language of projective modules, as presented in [24] and 
references therein, and give the explicit map between this formulation and the more elementary formulation in $[39,14]$. I will also explain the notion of Morita equivalence $[60,24,67,68,49]$ applied to our specific case ${ }^{4}$. Finally I will end the chapter with a discussion the general theory of gauge transformations on the noncommutative torus and find an explicit gauge transformation that trivializes one of the transition functions. With trivial transition functions, T-duality transformations take the standard form, allowing us to interpret the expectation value of the gauge field as the location of the D-strings on the dual torus.

In Chapter 4, I will include nonvanishing Chern-Simon couplings. The values of the Chern-Simon couplings are determined by the Ramond-Ramond moduli of the compactification. One finds perfect agreement between the transformation properties of the couplings derived from NCSYM or the auxiliary Type II string theory.

In Chapter 5, I will study the BPS spectrum corresponding to electric fluxes of the noncommutative supersymmetric Yang-Mills (NCSYM) gauge theory [22] compactified on a torus. This gives a description of the BPS spectrum of the DLCQ of M-theory compactified on a dual torus. Since the spectrum is invariant under the T-duality group $O(d, d \mid \mathbf{Z})$, where $d$ is the dimension of the compactification torus, one can first calculate the spectrum in the simplest case which corresponds to a NCSYM gauge theory on a trivial bundle. Then one can use

\footnotetext{
${ }^{4}$ For an expanded coverage of noncommutative geometry see [23] and for a brief description see [12].
} 
a duality transformation to rewrite the result in terms of the defining parameters of a dual theory on a nontrivial bundle. Alternatively one can also obtain this result directly by quantizing the free system of collective coordinates of the twisted $U(n)$ theory. To obtain the spectrum one has to mod out gauge equivalent configurations and show that the zero modes of the gauge field live on a compact space, a torus. In the classical case one can find a global gauge transformation whose sole effect is a shift in the zero mode of the gauge field. Then the electric fluxes which are the conjugate variables are integrally quantized. However, for a nonvanishing deformation parameter the gauge transformation also results in a finite space translation $[24,41,42,15]$. Then, just as for the electric charge of dyons [84], by a Witten-Olive type effect, the electric flux spectrum for states carrying momentum, contains an additional term proportional to the deformation parameter. The spectrum obtained is in agreement with the spectrum conjectured in the literature and obtained by imposing U-duality invariance.

\subsection{Path Integral Quantization of Poisson-Lie Groups and Reality Structures on Quantum Cotangent Bundles}

The Feynman path integral reveals in a geometric intuitive way the relation between classical and quantum dynamics. However there are few examples of path integral quantizations on compact phase spaces. These are interesting because they have finite dimensional Hilbert spaces. The simplest example is a phase space 
with the topology of a torus. A more interesting case is obtained by considering a phase space with the topology of the sphere $S_{2}$. Quantization of this gives the unitary representations of the lie algebra $s u(2)$. A path integral quantization is described in $[53,1]$. In Chapter $6, \mathrm{I}$ will present a generalization of this result to the unitary representation of $\mathcal{U}_{q}(s u(2))$.

Let $G$ be a Lie group. On the vector space $g^{*}$ dual to the Lie algebra $g$ of $G$ there is a natural Poisson structure. In terms of linear coordinates $e_{i}$ and $f_{i j}^{k}$, the structure constants of the group, it has the form

$$
\left\{e_{i}, e_{j}\right\}=f_{i j}^{k} e_{k}
$$

and it is known as the Lie-Kirillov-Kostant Poisson bracket. Its symplectic leaves are the orbits of the coadjoint action [47]. The quantization of this bracket is the universal enveloping algebra $\mathcal{U}(g)$ which is the associative algebra with generators $e_{i}$ and relations

$$
\left[e_{i}, e_{j}\right]=i \hbar f_{i j}^{k} e_{k}
$$

Quantization of the coadjoint orbits of a Lie group $G$ gives its unitary representations [47]. Various methods were used to quantize these symplectic leaves including geometric quantization and the Feynman path integral $[53,1]$. Note that the vector space $g^{*}$ can be thought of as an abelian group. The above picture can be generalized to include Poisson brackets on non-abelian groups $G^{*}$ usually called the dual Poisson-Lie groups. This will be extensively discussed later. Quantization of their symplectic leaves gives the unitary representations of the quantum 
group $\mathcal{U}_{q}(g)$. This can be summarized in the following diagram

$$
\begin{array}{ccc}
\operatorname{Fun}\left(G^{*}\right) & \rightarrow & F u n_{q}\left(G^{*}\right) \cong \mathcal{U}_{q}(g) \\
\uparrow & & \uparrow \\
F u n\left(g^{*}\right) & \rightarrow & \mathcal{U}(g)
\end{array}
$$

The quantization axis is horizontal, with classical Poisson-Lie groups on the left and their quantizations on the right. The vertical axis corresponds to deformation of the abelian case to the non-abelian case. Note that the abelian case can be obtained from the non-abelian case by looking at an infinitesimal neighborhood of the unit of the group, and rescaling coordinates appropriately. I will refer to the lower part of the picture already discussed in $[53,1]$ as the trivial case and to the upper part as the Poisson case.

Finally in Chapter 7 , I will introduce a new reality structure on the quantum cotangent bundle. Monodromy matrices representing the braid group [82], appearing in the WZNW-model, suggested that hidden quantum groups exist in these theories. Various approaches were used in an attempt to elucidate the origin of these hidden quantum groups. In [2, 4, 31, 3] using a Minkowski space-time lattice regularization, it was shown by explicit construction that the monodromies of the chiral components of the WZNW-model with Lie group $G$ and the local field satisfy the commutation relations of the $q$-deformed cotangent bundle $\mathrm{T}^{*} G_{q}$.

However, an apparent contradiction existed [4, 3], since the deformation parameter in the WZNW-model must be root of unity $q=\exp (i \pi / k+h)$, where $k$ is the level of the affine-Lie algebra and $h$ is the dual Coxeter number, and this is incompatible with the compact form of the quantum group. 
A solution to this problem was proposed in [5]. The main idea is to drop the strong requirement that the reality structure be compatible with quantum group comultiplication and only impose this requirement in the classical limit. Then a reality structure can be introduced, not on the quantum group itself, but rather on the quantum cotangent bundle.

Once the requirement of the compatibility of the reality structure with the comultiplication is dropped, one can introduce more than one reality structure. In Chapter $7, \mathrm{I}$ will introduce one such reality structure inspired by a particular type of non-compact WZNW-model. See for example [34] for a list of various circumstances under which this non-compact form occurs and also [25] where the non-compact form of appears as the Euclidean section of the model. These WZNW-models have the important property that the local field has the chiral decomposition $g=h h^{\dagger}$ where $h$ is the chiral field valued in $G$. Thus $g$ is a Hermitian positive defined matrix of unit determinant. I will show that

$$
g^{\dagger}=g
$$

is compatible with the algebra $\mathrm{T}^{*} G_{q}$ and extends the above anti-involution to the whole algebra. I emphasize that the reality structure introduced here is similar to the one discussed in [5] and is not related to the standard non-compact reality structure appearing in quantum groups for $q$ phase, and which is compatible with comultiplication.

For simplicity here I will not apply the reality structure directly in the WZNW- 
model, leaving this for a forthcoming paper, and instead I will just use it for the toy model of $[3,5]$, which essentially contains all the relevant degrees of freedom. These degrees of freedom are described by the same algebra as in the compact case but with a different reality structure. 


\section{Chapter 2}

\section{Super Yang-Mills on the Noncommutative Torus}

In the first section I will review the standard toroidal Matrix compactification leading to a SYM gauge theory on the dual torus. Then I will present the conjecture [24], that in the presence of nonvanishing NS antisymmetric moduli $B_{i j}$, the translation generators implementing the quotient condition do not commute, such that Matrix compactification leads to a noncommutative super Yang-Mills gauge theory on a dual noncommutative torus.

In Section 2.3, I will study adjoint quantum bundles on noncommutative tori of arbitrary dimension which admit a constant curvature which is not valued in the $s u(n)$ subalgebra and have transition functions of a special simple form.

In Section $2.4 \mathrm{I}$ will show how to expand the sections of the adjoint bundle of a $U(n)$ gauge theory in terms of matrix valued functions on a dual noncommutative torus. The dual deformation parameter $\Theta^{\prime}$ lies on the same $S O(d, d \mid \mathbf{Z})$ orbit as the original $\Theta$. I will perform most of the calculations on tori of arbitrary dimension, but later I will concentrate on the two and three-tori.

In Section 2.5, I describe the quantum bundles corresponding to the two dimensional compactification and rewrite some of the known two dimensional relations in a form that admits immediate generalization to higher dimensions. I will also 
give the solution for arbitrary adjoint bundles over three dimensional tori.

In Section 2.6, I will consider the noncommutative SYM action on a twisted quantum bundle after a brief description of the quantum integral.

Finally in Appendix A I will prove a theorem showing that the chiral spinor representations of $S O(d, d \mid \mathbf{Z})$ are integral, and also show that the spinor representation of $S O(3,3 \mid \mathbf{Z})$ is in fact $S L(4, \mathbf{Z})$.

\subsection{Matrix Compactification}

In this section I will present a review of Matrix theory compactification. In the limit of large string mass the dynamics of $n$ Do branes, in uncompactified spacetime, is determined by the maximally supersymmetric Matrix action $[20,32,8]$,

$$
\mathcal{S}^{D 0}=\frac{1}{2 g_{S}} \int d t \operatorname{tr}\left(\sum_{M} \dot{X}^{M} \dot{X}^{M}+\frac{1}{(2 \pi)^{2}} \sum_{M<N}\left[X^{M}, X^{N}\right]\left[X^{M}, X^{N}\right]+\text { fermions }\right) .
$$

This action is obtained by dimensional reduction of the ten dimensional $\mathcal{N}=1$ SYM gauge theory. Alternatively one could work with the IKKT functional [44] obtained by dimensionally reducing, in all directions including time, the Euclidean ten dimensional SYM action.

The compactification of Matrix theory on a $d$-dimensional torus is obtained by considering an infinite number of D0 branes living on $\mathbf{R}^{d}$, the covering space of the torus, and then imposing the following quotient conditions $[9,78]$.

$$
\begin{aligned}
\mathcal{U}_{i}^{-1} X^{I} \mathcal{U}_{i} & =2 \pi e_{i}^{I}+X^{I}, i, I=1, \ldots, d \\
\mathcal{U}_{i}^{-1} X^{a} \mathcal{U}_{i} & =X^{a}, a=d+1, \ldots, 9
\end{aligned}
$$




$$
\mathcal{U}_{i}^{-1} \psi \mathcal{U}_{i}=\psi
$$

The last equation in (2.1) contains the quotient condition for fermions. Here $I$ runs over the compact directions, and the $e_{i}^{I}$ form a basis defining the compactification lattice. The $\mathcal{U}_{i}$ 's are unitary operators. One can define new matrix coordinates

$$
X^{i}=e_{I}^{i} X^{I}
$$

which obey the simpler quotient conditions

$$
\mathcal{U}_{i}^{-1} X^{j} \mathcal{U}_{i}=2 \pi \delta_{i}^{j}+X^{j}
$$

In terms of the new variables the action takes the form

$$
\begin{gathered}
\mathcal{S}^{D 0}=\frac{1}{2 g_{S}} \int d t \operatorname{Tr}\left(G_{i j} \dot{X}^{i} \dot{X}^{j}+\frac{1}{2} \frac{1}{(2 \pi)^{2}} G_{i j} G_{k l}\left[X^{i}, X^{k}\right]\left[X^{j}, X^{l}\right]+\right. \\
\sum_{a} \dot{X}^{a} \dot{X}^{a}+\frac{1}{(2 \pi)^{2}} \sum_{a} G_{i j}\left[X^{i}, X^{a}\right]\left[X^{j}, X^{a}\right]+ \\
\left.\frac{1}{(2 \pi)^{2}} \sum_{a<b}\left[X^{a}, X^{b}\right]\left[X^{a}, X^{b}\right]+\text { fermions }\right),
\end{gathered}
$$

where I have introduced the metric $G_{i j}=\sum_{I} e_{i}^{I} e_{j}^{I}$. In (2.3), the trace over infinite dimensional matrices is formally divided by the infinite order the quotient group $\mathbf{Z}^{d}$

The original solution of the quotient condition assumed that the translation operators commute

$$
\left[\mathcal{U}_{i}, \mathcal{U}_{j}\right]=0
$$

The standard way to solve (2.2) is to introduce an auxiliary Hilbert space on which $X^{i}$ 's and $\mathcal{U}_{i}$ 's act. In the simplest case this is taken to be the space of functions 
on a $d$-dimensional torus taking values in $\mathrm{C}^{n}$. Then if one lets the $\mathcal{U}_{i}$ 's be the generators of the algebra of functions on the torus

$$
\mathcal{U}_{i}=e^{i \sigma_{i}}
$$

where $\sigma_{i}$ are coordinates on the covering space of the torus, the $X^{i}$ 's satisfying (2.2) must be covariant derivatives

$$
X^{j}=-2 \pi i D^{j}=-2 \pi i\left(\partial^{j}-i A^{j}\left(\mathcal{U}_{k}\right)\right)
$$

The partial derivative is with respect to $\sigma_{j}$, and $A^{j}$ are $n$-dimensional hermitian matrices. The action (2.3) can be rewritten as a $d$-dimensional SYM action, by replacing the $X^{i}$ 's with covariant derivatives as above, and rewriting the trace over the infinite dimensional matrices as

$$
\operatorname{Tr}=\int \frac{d^{d} \sigma}{(2 \pi)^{d}} \operatorname{tr}
$$

Here $\operatorname{tr}$ is an $n$-dimensional trace, and the new coordinates $\sigma_{i}$ are to be integrated from zero to $2 \pi$. The action becomes

$$
\begin{aligned}
\mathcal{S}^{D 0}= & \frac{(2 \pi)^{2-d}}{4 g_{S} \sqrt{\operatorname{det}\left(G^{i j}\right)}} \int d t \int d^{d} \sigma \sqrt{\operatorname{det}\left(G^{i j}\right)} \operatorname{tr}\left(G_{\mu \nu} G_{\xi \rho}\left[D^{\mu}, D^{\xi}\right]\left[D^{\nu}, D^{\rho}\right]-\right. \\
& \left.\sum_{a} G_{\mu \nu}\left[D^{\mu}, X^{a}\right]\left[D^{\nu}, X^{a}\right]+\sum_{a<b}\left[X^{a}, X^{b}\right]\left[X^{a}, X^{b}\right]+\text { fermions }\right),
\end{aligned}
$$

where the scalar fields $X^{a}$ have been rescaled by a factor of $2 \pi . I$ have written the action in standard form ${ }^{1}$ so that one can read off the SYM gauge coupling

$$
g_{S Y M}^{2}=g_{S}(2 \pi)^{d-2} \sqrt{\operatorname{det}\left(G^{i j}\right)}
$$

\footnotetext{
${ }^{1}$ Note that the positions of all the indices are switched. For example the metric has upper indices. This just reflects the performed T-duality under which the metric is replaced with the
} 
Thus the gauge coupling $g_{S Y M}^{2}$ equals the string coupling on the T-dual torus. The square root factor accounts for the expected dilaton shift under T-duality.

Following [24] I will consider the general case when the unitary operators $\mathcal{U}_{i}$ do not commute. Consistency of the quotient conditions requires that the $\mathcal{U}_{i}$ 's must commute up to a phase

$$
\mathcal{U}_{i} \mathcal{U}_{j}=e^{-2 \pi i \Theta_{i j}} \mathcal{U}_{j} \mathcal{U}_{i}
$$

Connes, Douglas and Schwarz conjectured that the deformation parameters $\Theta$ correspond to certain moduli of the compactification of the DLCQ of M theory on tori. If $\gamma^{i j-}$ represents a three cycle wrapped around the transversal directions $x^{i}$ and $x^{j}$ and the light cone direction $x^{-}$, then

$$
\Theta_{i j}=\frac{1}{(2 \pi)^{3}} \int_{\gamma^{i j-}} C
$$

where $C$ is the antisymmetric three form of eleven dimensional supergravity. Written in terms of the auxiliary type IIA string theory variables,

$$
\Theta_{i j}=\frac{1}{(2 \pi)^{2}} \int_{\gamma^{i j}} B
$$

where $B$ is the NS two form.

Next I will give a heuristic explanation of the noncommutativity of the translation generators (2.6). Consider for simplicity compactification on $T^{2}$ and assume that there is only one D0-brane. Then the covering space depicted in figure 2.1 inverse metric. Another way to understand the index position is that T-duality is a canonical transformation which exchanges coordinates and momenta and therefore reverses the index structure. 
contains an infinite number of $T^{2}$ cells labeled by two integers. The matrix element $X_{p q}^{i}$ is associated to the oriented string starting on the D0-brane at $q=\left(q_{1}, q_{2}\right)$ and ending on the D0-brane at $p=\left(p_{1}, p_{2}\right)$. I will define the translation operators such that their matrix elements are given by

$$
\begin{aligned}
& \left(\mathcal{U}_{1}\right)_{p q}=\delta_{p_{1}, q_{1}-1} \delta_{p_{2}, q_{2}}, \\
& \left(\mathcal{U}_{2}\right)_{p q}=\delta_{p_{1}, q_{1}} \delta_{p_{2}, q_{2}-1} .
\end{aligned}
$$

The translation generators then commute since each acts trivially in one subspace. Next consider the effect of turning on the modulus $B=B_{12} d x^{1} d x^{2}$. Since the covering space is topologically trivial and $B_{12}$ is constant one can eliminate it by a gauge transformation

$$
B^{\prime}=B+d \Lambda
$$

where for example $\Lambda=B_{12} x_{2} d x^{1}$. Eliminating $B$ comes at a price. One must modify the operators $\mathcal{U}_{i}$ implementing the periodicity condition.

Just as the wave function of a charged particle changes under electromagnetic gauge transformations

$$
\psi^{\prime}(x)=e^{i \lambda(x)} \psi(x)
$$

the wave functional of strings $\Psi[\Gamma]$, where $\Gamma$ is the curve where the string is located, must transform under the gauge transformation (2.8) as

$$
\Psi^{\prime}[\Gamma]=e^{\frac{i}{2 \pi} \int_{\Gamma} \Lambda} \Psi[\Gamma]
$$

For the choice of $\Lambda$ made above the wave functional of each string acquires the 


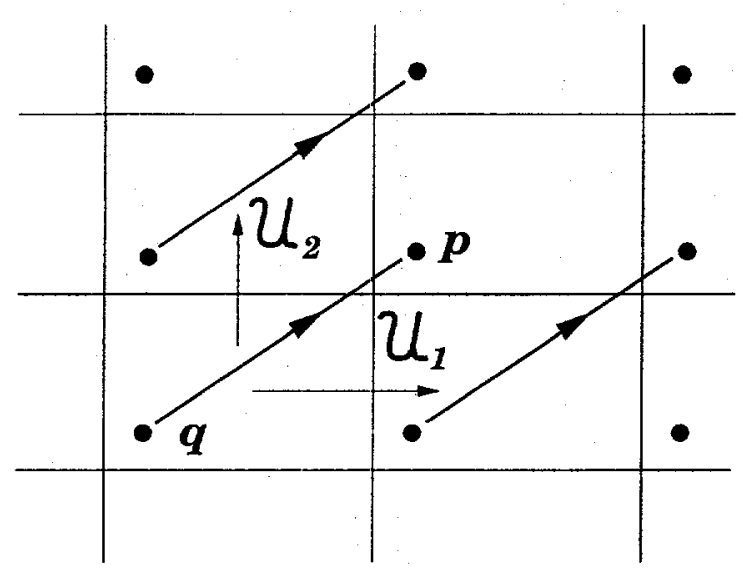

Figure 2.1: The covering space of $T^{2}$ with one D0-brane per cell

phase $\exp \left(i / 2 \pi B_{12} \int x^{2} d x^{1}\right)$. The exponent is just the area under the string so the relative phase of strings related by a translation in the $x^{1}$ direction vanishes. For the $x^{2}$ direction the relative phase is $\exp \left(2 \pi i \Theta_{12}\left(p_{1}-q_{1}\right)\right)$. This requires a redefinition of the translation generators

$$
\begin{aligned}
& \left(\mathcal{U}_{1}\right)_{p q}=\delta_{p_{1} q_{1}-1} \delta_{p_{2} q_{2}} \\
& \left(\mathcal{U}_{2}\right)_{p q}=e^{-2 \pi i \Theta_{12} q_{1}} \delta_{p_{1} q_{1}} \delta_{p_{2} q_{2}-1}
\end{aligned}
$$

Then a direct computations shows that the $\mathcal{U}_{i}$ 's satisfy (2.6).

In the noncommutative case it is convenient to introduce another set of translation operators $U_{i}$ which satisfy

$$
U_{i} U_{j}=e^{2 \pi i \Theta_{i j}} U_{j} U_{i} .
$$

The $U_{i}$ 's generate the algebra of functions on a quantum torus. I will denote this algebra, $\mathcal{A}_{\Theta}$. Also note that the $\mathcal{U}_{i}$ 's are the generators of $\mathcal{A}_{(-\Theta)}$. An expanded discussion of this and other issues in noncommutative geometry can be found 
in $[23,12]$.

This algebra can be realized as a subalgebra of the quantum plane algebra, which is generated by $\sigma_{i}$ satisfying

$$
\left[\sigma_{i}, \sigma_{j}\right]=-2 \pi i \Theta_{i j}
$$

Then one can realize the generators of $\mathcal{A}_{\Theta}$ as

$$
U_{i} \stackrel{\text { def }}{=} e^{i \sigma_{i}}
$$

To realize the $\mathcal{U}_{i}$ generators I will also introduce partial derivatives satisfying ${ }^{2}$

$$
\left[\partial^{i}, \sigma_{j}\right]=\delta_{j}^{i},\left[\partial^{i}, \partial^{j}\right]=0
$$

Now one can write the $\mathcal{U}_{i}$ generators as

$$
\mathcal{U}_{i}=e^{i \sigma_{i}-2 \pi \Theta_{i j} \partial^{j}}
$$

Note that both $\sigma_{i}$ and $\partial^{i}$ act as translation generators on the $\sigma_{i}$ 's, and the exponent in the $\mathcal{U}_{i}$ 's is just the linear combination that commutes with all the $\sigma_{i}$ 's. Thus

$$
\left[\mathcal{U}_{i}, U_{j}\right]=0
$$

For vanishing $\Theta$ one sees that $U_{i}$ and $\mathcal{U}_{i}$ coincide.

The simplest example of solutions of the quotient conditions (2.2) are quantum connections on trivial bundles

$$
X^{j}=-2 \pi i D^{j}=-2 \pi i\left(\partial^{j}-i A^{j}\left(U_{k}\right)\right) .
$$

\footnotetext{
${ }^{2}$ Just as in the classical case, one can also introduce quantum exterior forms $d \sigma_{i}$, which anti-commute with each other and commute with all other variables.
} 
In the noncommutative case the matrix elements of $A^{j}$ are elements of $\mathcal{A}_{\ominus}$. Again using the representation (2.12) of $X^{i}$ in the Matrix model action one obtains a NCSYM action [22]. However I will postpone writing this action until I study more general solutions which are connections on nontrivial bundles.

For the commutative case, matrix compactification on $T^{d}$ results in a SYM gauge theory in $d+1$ dimensions on the dual torus. In the limit when the size of

the original torus vanishes the dual torus becomes $\mathbf{R}^{d}$, therefore one obtain the opposite of dimensional reduction. If one starts from a Euclidean 10-dimensional SYM and dimensionally reduces in all directions including the Euclidean time one obtains the IKKT [44] functional. Matrix compactification of one direction in the IKKT functional results in the finite temperature action of the original theory (2.3).

\subsection{Twisted Quantum Bundles on $T^{2}$}

There exist more general solutions of the quotient condition (2.2) which are connections on twisted bundles. They correspond to compactification of the DLCQ of M-theory in the presence of transversely wrapped membranes. Again the solution is a sum of two terms, a constant curvature connection $\nabla^{i}$ and a fluctuating part

$$
\begin{aligned}
X^{i} & =-2 \pi i\left(\nabla^{i}-i A^{i}\left(Z_{j}\right)\right), \\
X^{a} & =X^{a}\left(Z_{i}\right)
\end{aligned}
$$




$$
\psi=\psi\left(Z_{i}\right)
$$

Here the $Z_{i}$ 's are $n \times n$ matrices with operator entries and, just like the $\tilde{U}_{i}$ 's for the trivial bundle, commute with the $U_{i}^{\prime}$ 's, but now are sections of the twisted bundle whose exact form will be discussed shortly. However, while for the trivial bundle $A^{i}, X^{a}$ and the spinorial components of $\psi$ are $n \times n$ matrix functions, in (2.13) $A^{i}, X^{a}$ and the components of $\psi$ are one-dimensional functions but with matrix arguments. Later, this will allow us to establish a relationship between a SYM on a twisted $U(n)$ bundle and one on a $U(1)$ bundle.

In this chapter we concentrate for simplicity on the two dimensional case. Following [39], up to a gauge transformation the constant curvature connection can be written as

$$
\nabla^{1}=\partial^{1}, \nabla^{2}=\partial^{2}-i f \sigma_{1}
$$

where $f$ is the constant field strength

$$
\left[\nabla^{1}, \nabla^{2}\right]=-i f
$$

Such a gauge field can only exist in a non-trivial bundle. One can introduce transition functions $\Omega_{i}$ such that the sections of the fundamental bundle satisfy the twisted boundary conditions

$$
\begin{aligned}
& \Phi\left(\sigma_{1}+2 \pi, \sigma_{2}\right)=\Omega_{1}\left(\sigma_{1}, \sigma_{2}\right) \Phi\left(\sigma_{1}, \sigma_{2}\right), \\
& \Phi\left(\sigma_{1}, \sigma_{2}+2 \pi\right)=\Omega_{2}\left(\sigma_{1}, \sigma_{2}\right) \Phi\left(\sigma_{1}, \sigma_{2}\right) .
\end{aligned}
$$

Similarly the adjoint sections satisfy

$$
\Psi\left(\sigma_{1}+2 \pi, \sigma_{2}\right)=\Omega_{1}\left(\sigma_{1}, \sigma_{2}\right) \Psi\left(\sigma_{1}, \sigma_{2}\right) \Omega_{1}\left(\sigma_{1}, \sigma_{2}\right)^{-1},
$$




$$
\Psi\left(\sigma_{1}, \sigma_{2}+2 \pi\right)=\Omega_{2}\left(\sigma_{1}, \sigma_{2}\right) \Psi\left(\sigma_{1}, \sigma_{2}\right) \Omega_{2}\left(\sigma_{1}, \sigma_{2}\right)^{-1}
$$

Consistency of the transition functions of the bundle requires that

$$
\Omega_{1}\left(\sigma_{1}, \sigma_{2}+2 \pi\right) \Omega_{2}\left(\sigma_{1}, \sigma_{2}\right)=\Omega_{2}\left(\sigma_{1}+2 \pi, \sigma_{2}\right) \Omega_{1}\left(\sigma_{1}, \sigma_{2}\right)
$$

This relation is known in the mathematical literature as the cocycle condition. The covariant derivatives transform just as the adjoint sections

$$
\begin{aligned}
& \nabla^{i}\left(\sigma_{1}+2 \pi, \sigma_{2}\right)=\Omega_{1}\left(\sigma_{1}, \sigma_{2}\right) \nabla^{i}\left(\sigma_{1}, \sigma_{2}\right) \Omega_{1}\left(\sigma_{1}, \sigma_{2}\right)^{-1} \\
& \nabla^{i}\left(\sigma_{1}, \sigma_{2}+2 \pi\right)=\Omega_{2}\left(\sigma_{1}, \sigma_{2}\right) \nabla^{i}\left(\sigma_{1}, \sigma_{2}\right) \Omega_{2}\left(\sigma_{1}, \sigma_{2}\right)^{-1}
\end{aligned}
$$

A particular solution for the transition functions compatible with the constant curvature connection (2.14) and satisfying the cocycle condition is given by

$$
\Omega_{1}=e^{i m \sigma_{2} / n} U, \Omega_{2}=V
$$

where $U, V$ are $n \times n$ unitary matrices satisfying

$$
U V=e^{-2 \pi i m / n} V U
$$

Using the representation given in [39] one has

$$
U_{k l}=e^{2 \pi i k m / n} \delta_{k, l}, \quad V_{k l}=\delta_{k+1, l}
$$

where the subscripts are identified with period $n$.

One can express the above matrices in terms of the standard 't Hooft matrices [80] denoted here by $U^{\prime}$ and $V^{\prime}$ and satisfying

$$
U^{\prime} V^{\prime}=e^{-2 \pi i / n} V^{\prime} U^{\prime}, \quad U^{\prime n}=V^{\prime n}=1
$$


The relation is given by

$$
U=e^{2 \pi i m / n} U^{\prime m}, \quad V=V^{\prime}
$$

The phase in (2.19) is due to the nonstandard definition of $U$ used in [39]. This has certain advantages but similar phases will appear when comparing the results of [39] with similar results where the standard 't Hooft matrices were used. I also introduce a unitary matrix $K$ which changes the representation so that $V^{\prime}$ is diagonal, and satisfies

$$
K U^{\prime} K^{-1}=V^{\prime-1}, \quad K V^{\prime} K^{-1}=U^{\prime}
$$

Note that $n$ is quantized since one is considering a $U(n)$ gauge theory and $m$ is quantized since the magnetic flux $f$ through $T_{2}$ is quantized

$$
2 \pi f=\frac{m}{n+m \theta}
$$

where $\theta=\Theta_{12}$. In M-theory $m$ is the transversal membrane wrapping number.

One can solve the boundary conditions (2.15) for the fundamental sections as in [39] generalizing a previous result for $m=1$ in the commutative case presented in [33]. Using the ordered exponential explained below, the general solution has the form

$$
\Phi_{k}\left(\sigma_{1}, \sigma_{2}\right)=\sum_{s \in \mathbf{Z}} \sum_{j=1}^{m} E\left(\frac{m}{n}\left(\frac{\sigma_{2}}{2 \pi}+k+n s\right)+j, i \sigma_{1}\right) \widehat{\phi}_{j}\left(\frac{\sigma_{2}}{2 \pi}+k+n s+\frac{n j}{m}\right) .
$$

The ordered exponential [39] is defined for two variables whose commutator is a $c$-number

$$
E(A, B)=\frac{1}{1-[A, B]} \sum_{l=0}^{\infty} \frac{1}{l !} A^{l} B^{l}
$$


The normalization is such that

$$
E(-B, A) E(A, B)=1
$$

and it has the following desirable properties similar to the usual exponential

$$
\begin{aligned}
& E(A+c, B)=E(A, B) e^{c B}, \\
& E(A, B+c)=e^{c A} E(A, B) .
\end{aligned}
$$

The $\widehat{\phi}_{j}$ functions are defined on the whole real axis and are unrestricted except for the behavior at infinity. They should be considered as vectors in a Hilbert space on which all the elements of the algebra are represented.

Next I will explain in some detail how to obtain this result. First define

$$
\phi\left(\sigma_{1}, \sigma_{2}\right) \stackrel{\text { def }}{=} \Phi_{k}\left(\sigma_{1}, \sigma_{2}-2 \pi(k-1)\right) .
$$

The second boundary condition (2.15) implies that the definition of $\phi$ is consistent, i.e. $k$-independent. Using $V^{n}=1$ one also finds that $\phi$ is a periodic function in $\sigma_{2}$

$$
\phi\left(\sigma_{1}, \sigma_{2}+2 \pi n\right)=\phi\left(\sigma_{1}, \sigma_{2}\right) .
$$

The other boundary condition gives

$$
\phi\left(\sigma_{1}+2 \pi, \sigma_{2}\right)=e^{i m\left(\sigma_{2}+2 \pi\right) / n} \phi\left(\sigma_{1}, \sigma_{2}\right) .
$$

It is convenient to separate out a factor to eliminate the above twist

$$
\phi\left(\sigma_{1}, \sigma_{2}\right)=f\left(\sigma_{1}, \sigma_{2}\right) \check{\phi}\left(\sigma_{1}, \sigma_{2}\right)
$$


and to require a simpler periodicity condition for $\check{\phi}$

$$
\check{\phi}\left(\sigma_{1}+2 \pi, \sigma_{2}\right)=\check{\phi}\left(\sigma_{1}, \sigma_{2}\right)
$$

Then the function $f$ must satisfy

$$
f\left(\sigma_{1}+2 \pi, \sigma_{2}\right)=e^{i m\left(\sigma_{2}+2 \pi\right) / n} f\left(\sigma_{1}, \sigma_{2}\right) .
$$

This is satisfied exactly for

$$
f\left(\sigma_{1}, \sigma_{2}\right)=E\left(\frac{m}{n}\left(\frac{\sigma_{2}}{2 \pi}+1\right), i \sigma_{1}\right)
$$

where the ordered exponential defined above was used in the right hand side. One can Fourier transform $\check{\phi}$ in $\sigma_{1}$

$$
\check{\phi}\left(\sigma_{1}, \sigma_{2}\right)=\sum_{p \in \mathbf{Z}} e^{i p \sigma_{1}} \phi_{p}\left(\sigma_{2}\right)
$$

and using the property (2.21) of the ordered exponential one obtains

$$
\phi\left(\sigma_{1}, \sigma_{2}\right)=\sum_{p \in \mathbf{Z}} E\left(\frac{m}{n}\left(\frac{\sigma_{2}}{2 \pi}+1\right)+p, i \sigma_{1}\right) \phi_{p}\left(\sigma_{2}\right) .
$$

Let $p=m s+j$ with $j=1, \ldots, m$ and $s$ is an integer. Then the solution can be written as

$$
\phi\left(\sigma_{1}, \sigma_{2}\right)=\sum_{s \in \mathbf{Z}} \sum_{j=1}^{m} E\left(\frac{m}{n}\left(\frac{\sigma_{2}}{2 \pi}+1\right)+m s+j, i \sigma_{1}\right) \phi_{s, j}\left(\sigma_{2}\right),
$$

where $\phi_{s, j} \stackrel{\text { def }}{=} \phi_{m s+j}$. Periodicity in $\sigma_{2}$ then implies $\phi_{s-1, j}\left(\sigma_{2}+2 \pi n\right)=\phi_{s, j}\left(\sigma_{2}\right)$ so that using this recursively one has $\phi_{s, j}\left(\sigma_{2}\right)=\phi_{0, j}\left(\sigma_{2}+2 \pi n s\right)$. Finally after defining $\tilde{\phi}_{j}(x) \stackrel{\text { def }}{=} \phi_{0, j}(2 \pi(x-1))$ one obtains

$$
\Phi_{k}\left(\sigma_{1}, \sigma_{2}\right)=\sum_{s \in \mathbf{Z}} \sum_{j=1}^{m} E\left(\frac{m}{n}\left(\frac{\sigma_{2}}{2 \pi}+k+n s\right)+j, i \sigma_{1}\right) \tilde{\phi}_{j}\left(\frac{\sigma_{2}}{2 \pi}+k+n s\right) .
$$


This is the result mentioned above up to another redefinition

$$
\tilde{\phi}_{j}(x)=\widehat{\phi}_{j}\left(x+\frac{n}{m} j\right)
$$

While the solutions for the sections of the fundamental bundle given in [39] are suitable for showing the equivalence to the projective modules of [24] as will be discussed in Section 3.3, the appearance of the ordered exponential is somewhat inconvenient. Using the special form of the transition functions one can rewrite the solution in an equivalent but simpler form. The transition functions in this gauge do not contain $\sigma_{1}$ and it is convenient to order all $\sigma_{1}$ to the right in the solution. Using $V^{n}=1$ in the second condition (2.15) one can express all $n$ components of $\Phi$ in terms of a single function with period $2 \pi n$ in $\sigma_{2}$. After Fourier transforming in $\sigma_{2}$ and imposing both boundary conditions (2.15) one obtains the general solution

$$
\Phi_{k}\left(\sigma_{1}, \sigma_{2}\right)=\sum_{p \in \mathbf{Z}} e^{2 \pi i\left(\sigma_{2} / 2 \pi+k\right) p / n} e^{2 \pi i\left(\sigma_{1} / 2 \pi-p / m\right) m / n} \widehat{\varphi}_{p}\left(\sigma_{1} / 2 \pi-p / m\right)
$$

where only $m$ of the $\hat{\varphi}_{p}$ functions are independent, since

$$
\widehat{\varphi}_{p+m}(x)=\widehat{\varphi}_{p}(x)
$$

Using the same technique one can show that an arbitrary adjoint section has the following expansion

$$
\Psi\left(\sigma_{1}, \sigma_{2}\right)=\sum_{s, t \in \mathbf{Z}} c_{s t} Z_{1}^{s} Z_{2}^{-t}
$$

Here $c_{s, t}$ are $c$-numbers and

$$
Z_{1}=e^{i \sigma_{1} /(n+m \theta)} V^{b}, \quad Z_{2}=e^{i \sigma_{2} / n} U^{-b}
$$


where $b$ is an integer, such that one can find another integer $a$ satisfying $a n-b m=$ 1. For $n$ and $m$ relatively prime one can always find integer solutions to this equation. Again, let me emphasize that the $Z_{i}$ 's commute with the $U_{i}$ 's. They are generators of the algebra of functions on a new quantum torus

$$
Z_{1} Z_{2}=e^{2 \pi i \theta^{\prime}} Z_{2} Z_{1}
$$

where $\theta^{\prime}$ is obtained by an $S L(2, \mathbf{Z})$ fractional transformation from $\theta$

$$
\theta^{\prime}=\frac{a \theta+b}{m \theta+n}
$$

Now I will outline how to obtain this result. Note first that

$$
\Psi\left(\sigma_{1}+2 \pi n, \sigma_{2}\right)=\Omega_{1}^{n} \Psi\left(\sigma_{1}, \sigma_{2}\right) \Omega_{1}^{-n}=\Psi\left(\sigma_{1}-2 \pi \theta m, \sigma_{2}\right) .
$$

In the last equality I used the fact that $U^{n}=1$, and the exponential formula to shift $\sigma_{1}$. Using both boundary conditions one has

$$
\begin{gathered}
\Psi\left(\sigma_{1}+2 \pi(n+m \theta), \sigma_{2}\right)=\Psi\left(\sigma_{1}, \sigma_{2}\right), \\
\Psi\left(\sigma_{1}, \sigma_{2}+2 \pi n\right)=\Psi\left(\sigma_{1}, \sigma_{2}\right) .
\end{gathered}
$$

Next, expand the section as

$$
\Psi\left(\sigma_{1}, \sigma_{2}\right)=\sum_{s, t \in \mathbf{Z}} e^{i s \sigma_{1} /(n+m \theta)} e^{-i t \sigma_{2} / n} \Psi_{s, t},
$$

where $\Psi_{s, t}$ is a $n \times n$ matrix and can be expanded as

$$
\Psi_{s, t}=\sum_{i=i_{0}}^{n+i_{0}} \sum_{j=j_{0}}^{n+j_{0}} c_{s, t, i, j} V^{\prime i} U^{\prime j}
$$


Here $i_{0}, j_{0}$ are two arbitrary integers, allowing us to freely shift the summation limits assuming that $c_{s, t, i+n, j}=c_{s, t, i, j+n}=c_{s, t, i, j}$. Then one can obtain further restrictions on the $c_{s, t, i, j}$ coefficients using the boundary conditions (2.16). For example using the first equation (2.16) and comparing like coefficients in the Fourier expansion one has

$$
c_{s, t, i, j} e^{2 \pi i s /(n+m \theta)}=c_{s, t, i, j} e^{-2 \pi i m i / n} e^{-2 \pi i s m \theta /[n(n+\theta)]} .
$$

From this and the similar relation obtained by imposing the second equation (2.16) one sees that $c_{s, t, i, j}$ vanish unless $(s+m i) / n=k$ and $(t+j) / n=s$ for $k$ and $s$ two integers. These equations have multiple solutions. However, if $(i, j)$ and $\left(i^{\prime}, j^{\prime}\right)$ are two solutions then $i-i^{\prime} \in n \mathbf{Z}$ and $j-j^{\prime} \in n \mathbf{Z}$. This ensures that only one term survives in the sum (2.23) over $i$ and $j$. Choosing for later convenience $i_{0}=s b$ and $j_{0}=m b t$ one has

$$
\Psi\left(\sigma_{1}, \sigma_{2}\right)=\sum_{s, t \in \mathbf{Z}} e^{i s \sigma_{1} /(n+m \theta)} e^{-i t \sigma_{2} / n} \sum_{i=s b}^{n+s b} \sum_{j=m b t}^{n+m b t} c_{s, t, i, j} V^{\prime i} U^{\prime j}
$$

Since $n$ and $m$ are relatively prime let $a, b \in \mathbf{Z}$ such that $a n-b m=1$. Then

$$
k=a s, l=a t, i=b s, j=m b t
$$

is an integer solution inside the $i$ and $j$ summation range. Dropping the $i, j$ indices since they are determined by $s$ and $t$ one has

$$
\Psi\left(\sigma_{1}, \sigma_{2}\right)=\sum_{s, t \in \mathbf{Z}} c_{s, t}\left(e^{i \sigma_{1} /(n+m \theta)} V^{\prime b}\right)^{s}\left(e^{i \sigma_{2} / n} U^{\prime-m b}\right)^{-t}
$$

which is just (2.22) after an additional phase redefinition of $c_{s, t}$ to accommodate the phase difference between $U$ and $U^{\prime m}$. 


\subsection{Twisted Quantum Bundles on Tori}

In this section I will construct quantum $U(n)$ bundles on $d$-dimensional noncommutative tori which admit constant curvature connections with vanishing $s u(n)$ curvature. This is done by finding explicit transition functions compatible with such a connection. I will employ a method which is a straightforward generalization of $[39,52]$. Using a gauge transformation the constant curvature connection can be brought into the form

$$
\nabla^{i}=\partial^{i}+i F^{i j} \sigma_{j}
$$

where $F$ is an antisymmetric matrix. This differs from the gauge used in the previous section, but is very convenient for the higher dimensional cases. Form now on it will be used through out the thesis unless otherwise stated. Define the constant curvature to be

$$
\mathcal{F}_{(0)}^{j k}=i\left[\nabla^{j}, \nabla^{k}\right]
$$

Then, using the commutation relations (2.11) one can calculate

$$
\mathcal{F}_{(0)}=(2 F+2 \pi F \Theta F) .
$$

In general, such a connection can only exist on a non-trivial bundle. One can introduce transition functions $\Omega_{i}$ such that the connection satisfies the twisted boundary conditions

$$
\nabla^{i}\left(\sigma_{m}+2 \pi \delta_{m}^{j}\right)=\Omega_{j}\left(\sigma_{m}\right) \nabla^{i}\left(\sigma_{m}\right) \Omega_{j}^{-1}\left(\sigma_{m}\right)
$$


One can try to find solutions for the transition functions of the form

$$
\Omega_{j}=e^{i P^{j l} \sigma_{l}} W_{j}
$$

where $P$ is an arbitrary constant $d$-dimensional matrix and the $W_{i}^{\prime}$ 's are constant, unitary $n$-dimensional matrices. The boundary conditions (2.25) imply the following equivalent relations

$$
\begin{gathered}
P=(1+2 \pi F \Theta)^{-1} 2 \pi F=2 \pi F(1+\Theta 2 \pi F)^{-1}, \\
2 \pi F=P(1-\Theta P)^{-1}=(1-P \Theta)^{-1} P
\end{gathered}
$$

Note that $P$ must be antisymmetric because of our gauge choice. Consistency of the transition functions of the bundle is the cocycle condition

$$
\Omega_{j}\left(\sigma_{m}+2 \pi \delta_{m}^{i}\right) \Omega_{i}\left(\sigma_{m}\right)=\Omega_{i}\left(\sigma_{m}+2 \pi \delta_{m}^{j}\right) \Omega_{j}\left(\sigma_{m}\right)
$$

In our case it implies

$$
W_{i} W_{j}=e^{-2 \pi i M^{i j} / n} W_{j} W_{i}
$$

where the antisymmetric matrix $M$ is given by

$$
M=n(2 P-P \Theta P)
$$

By taking the determinant of both sides of (2.27) one finds that $M$ must have integer entries. In the classical case $M^{i j}$ corresponds to the value of the first Chern class on the $(i j)$ two-cycle of the torus. In the auxiliary Type IIA string theory, $M$ is interpreted as D2 brane winding. This interpretation remains true in the quantum case. 
Let $q$ be the greatest common divisor of $n$ and the nonvanishing entries of $M$

$$
q=\operatorname{gcd}\left(n, M^{i j}\right)
$$

Next one defines $\tilde{n}$ and $\widetilde{M}$ which have relatively prime entries

$$
n=q \tilde{n}, \quad M=q \widetilde{M} .
$$

It is convenient to consider $W_{i}$ 's which have block diagonal form with $q$ identical blocks along the diagonal

$$
W_{i}=\left(\begin{array}{ccc}
\widetilde{W}_{i} & & \\
& \ddots & \\
& & \widetilde{W}_{i}
\end{array}\right) .
$$

Here $\widetilde{W}_{i}$ are $\tilde{n}$-dimensional matrices. Alternatively one can write this in tensor product notation

$$
W_{i}=I_{q} \otimes \widetilde{W}_{i}
$$

The transition functions are also block diagonal and can be written

$$
\Omega_{i}=I_{q} \otimes \omega_{i}
$$

To find explicit boundary conditions, following 't Hooft [81], one makes the ansatz

$$
\widetilde{W}_{i}=U^{a^{i}} V^{b^{i}}
$$

where $a^{i}$ and $b^{i}$ are integers and $U$ and $V$ are the clock and shift matrices $[80,81]$

$$
U_{k l}=e^{2 \pi i(k-1) / \tilde{n}} \delta_{k, l}, \quad V_{k l}=\delta_{k+1, l}, \quad k, l=1, \ldots, \tilde{n},
$$

and the subscripts are identified with period $\tilde{n}$. They satisfy

$$
U V=e^{-2 \pi i / \tilde{n}} V U
$$


Then (2.27) leads to the following relation

$$
\widetilde{M}^{i j}=\left(a^{i} b^{j}-b^{i} a^{j}\right) \bmod (\tilde{n})
$$

For two or three dimensional tori, one can find integers $a^{i}$ and $b^{i}$ such that (2.30) holds for arbitrary $M$, as will be shown in Section 2.5. In higher dimensional cases the ansatz is not sufficiently general to describe arbitrary bundles. In particular, one can always perform a change of lattice basis such that the only nonvanishing components of $M$ are $M^{d-1, d}=-M^{d, d-1}$, while in general, an arbitrary antisymmetric matrix can not be brought into such a form. Furthermore, for $d>3$, even in the commutative case, generic bundles do not admit connections with vanishing $s u(n)$ constant curvature. A more general construction could be obtained by allowing for an arbitrary constant curvature connection.

\subsection{Adjoint Sections on Twisted Bundles}

In this section I will analyze the structure of adjoint sections on twisted bundles. The scalar and fermion fields are examples of such sections. I will also write the connection as a sum of a constant curvature connection $\nabla^{i}$, and a fluctuating part $A^{i}$

$$
D^{i}=\nabla^{i}-i A^{i}
$$

Note that $A^{i}$ is also an adjoint section. Since it is the difference between two connections it transforms covariantly under gauge transformations. It should not be confused with a gauge potential. Adjoint sections are $n$-dimensional matrices 
with entries which are elements of the quantum plane algebra (2.11) and obey the twisted boundary conditions

$$
\Psi\left(\sigma_{i}+2 \pi \delta_{i}^{j}\right)=\Omega_{j} \Psi\left(\sigma_{i}\right) \Omega_{j}^{-1}
$$

Next I will try to find the general solution of (2.31) and write it in unconstrained form, reflecting the global properties of the bundle. First consider the simpler example of a $U(n)$ NCSYM on a trivial bundle over a two-torus. Since $\Omega_{i}=1$ one has

$$
\Psi=\sum_{a, b=1}^{n} E^{a b} \otimes\left[\sum_{i_{1} i_{2} \in \mathbf{Z}} \Psi_{i_{1} i_{2}}^{a b} U_{1}^{i_{1}} U_{2}^{i_{2}}\right]
$$

where $E^{a b}$ are $n$-dimensional matrices with one nonzero entry, $\left(E^{a b}\right)_{i j}=\delta_{i}^{a} \delta_{j}^{b}$, and $\Psi_{i_{1} i_{2}}^{a b}$ are $c$-numbers. In other words, each matrix element of the adjoint section is an arbitrary function on the quantum torus. Foe a twisted $U(n)$ bundle with magnetic flux $m$, such that $n$ and $m$ are relatively prime, one can show [24,39,52] that the adjoint sections have the expansion

$$
\Psi=\sum_{i_{1} i_{2} \in \mathbf{Z}} \Psi_{i_{1} i_{2}} Z_{1}^{i_{1}} Z_{2}^{i_{2}}
$$

where now the coefficients $\Psi_{i_{1} i_{2}}$ are $c$-numbers, and $Z_{i}$ are $n$-dimensional matrices with noncommutative entries satisfying

$$
Z_{1} Z_{2}=e^{2 \pi i \theta^{\prime}} Z_{2} Z_{1}
$$

Thus the $Z_{i}$ 's satisfy the commutation relations of a generators of the quantum torus. This shows that the set of sections is isomorphic to the set of functions on a dual torus, and is very similar to the set of adjoint sections of a $U(1)$ NCSYM 
theory. For two and three dimensional adjoint bundles with arbitrary magnetic fluxes, I will show that the general solution takes the form

$$
\Psi=\sum_{a b=1}^{q} E^{a b} \otimes\left[\sum_{i_{1} i_{2} \ldots i_{d} \in \mathbf{Z}} \Psi_{i_{1} i_{2} \ldots i_{d}}^{a b} Z_{1}^{i_{1}} Z_{2}^{i_{2}} \ldots Z_{d}^{i_{d}}\right], \quad d=2,3 .
$$

Here $E^{a b}$ are $q$ dimensional.

Begin by writing $\Psi$ in tensor notation

$$
\Psi\left(\sigma_{i}\right)=\sum_{a, b=1}^{q} E^{a b} \otimes \Psi^{a b}\left(\sigma_{i}\right)
$$

where $\Psi^{a b}\left(\sigma_{i}\right)$ are $\tilde{n}$-dimensional matrices with noncommutative entries. Imposing the boundary conditions (2.31) and using (2.28) one obtains

$$
\Psi^{a b}\left(\sigma_{i}+2 \pi \delta_{i}^{j}\right)=\omega_{j} \Psi^{a b}\left(\sigma_{i}\right) \omega_{j}^{-1}
$$

A less restrictive but very convenient constraint is obtained by shifting $\sigma_{i}$ by $2 \pi \tilde{n}$ using (2.33)

$$
\Psi^{a b}\left(\sigma_{i}+2 \pi \tilde{n} \delta_{i}^{j}\right)=\omega_{j}^{\tilde{n}} \Psi^{a b}\left(\sigma_{i}\right) \omega_{j}^{-\tilde{n}}
$$

In (2.34) all the matrix factors disappear since $U^{\tilde{n}}=V^{\tilde{n}}=1$. The $\sigma_{i}$ dependent exponential of (2.26) survives and acts like a translation operator due to the commutation relations (2.11). This implies the following periodicity relation

$$
\Psi^{a b}\left(\sigma_{i}+2 \pi \tilde{n}\left(Q^{-1}\right)_{i}^{j}\right)=\Psi^{a b}\left(\sigma_{i}\right)
$$

where

$$
Q^{-1}=1-P \Theta
$$


Next I will try to find solutions of the form

$$
Z_{i}=e^{i \sigma_{j} Q_{k}^{j} N_{i}^{k} / \bar{n}} U^{s_{i}} V^{t_{i}}, \quad i=1 \ldots d
$$

Here $s_{j}$ and $t_{j}$ are integers and the exponent was chosen so that it is compatible with the constraint (2.35) if the matrix $N$ has integer entries. One can show that $Z_{i}$ is compatible with the boundary conditions (2.33) if

$$
N_{j}^{i}=\left(b^{i} s_{j}-a^{i} t_{j}\right) \bmod (\tilde{n})
$$

where $a^{i}$ and $b^{i}$ are defined by (2.30). In the next two sections I will consider in detail the two and three dimensional cases, and find $a^{i}, b^{i}, s_{j}$ and $t_{j}$ such that (2.30) and (2.37) hold. Furthermore, for properly chosen integers $a^{i}, b^{i}, s_{j}$ and $t_{j}$, one can show that an arbitrary adjoint section can be expanded in terms of the $Z_{i}$ 's as in (2.32). For a proof of this statement in two dimensions see [52]. It is convenient to define another matrix which will be used shortly,

$$
L_{i j}=\left(s_{i} t_{j}-t_{i} s_{j}\right) \bmod (\tilde{n})
$$

In the remainder of this section I will calculate the commutation relations satisfied by the $Z_{i}$ 's and the constant curvature connection (2.24). Using their explicit form (2.36) one finds, after some matrix algebra,

$$
Z_{i} Z_{j}=e^{2 \pi i \Theta_{i j}^{\prime}} Z_{j} Z_{i}
$$

where

$$
\Theta^{\prime}=\tilde{n}^{-2} N^{T} Q^{T} \Theta Q N-\tilde{n}^{-1} L
$$


From (2.39) one can see that the algebra generated by the $Z_{i}$ 's is the algebra of functions on the quantum torus with deformation parameters given by $\Theta^{\prime}$. After some further matrix algebra and using the following identities,

$$
\begin{aligned}
Q & =1+2 \pi F \Theta, \\
Q^{2} & =1+2 \pi \mathcal{F}_{(0)} \Theta=(1-\widetilde{M} \Theta / \tilde{n})^{-1}, \\
Q^{T} \Theta & =\Theta Q
\end{aligned}
$$

one can rewrite $\Theta^{\prime}$ as a fractional transformation

$$
\Theta^{\prime}=\Lambda(\Theta) \stackrel{\text { def }}{=}(\mathcal{A} \Theta+\mathcal{B})(\mathcal{C} \Theta+\mathcal{D})^{-1}
$$

Here

$$
\Lambda=\left(\begin{array}{cc}
\mathcal{A} & \mathcal{B} \\
\mathcal{C} & \mathcal{D}
\end{array}\right),
$$

and the $d$-dimensional block matrices are given by

$$
\mathcal{A}=\tilde{n}^{-1}\left(N^{T}+L N^{-1} \widetilde{M}\right), \mathcal{B}=-L N^{-1}, \mathcal{C}=-N^{-1} \widetilde{M}, \mathcal{D}=\tilde{n} N^{-1}
$$

One can check that

$$
\mathcal{A}^{T} \mathcal{D}+\mathcal{C}^{T} \mathcal{B}=1, \mathcal{A}^{T} \mathcal{C}+\mathcal{C}^{T} \mathcal{A}=0, \mathcal{B}^{T} \mathcal{D}+\mathcal{D}^{T} \mathcal{B}=0
$$

and thus $\Lambda$ is an element of $O(d, d \mid \mathbf{R})$, i.e. it satisfies

$$
\Lambda^{T} J \Lambda=J
$$

where

$$
J=\left(\begin{array}{cc}
0 & I_{d} \\
I_{d} & 0
\end{array}\right)
$$


In the two and three dimensional examples that will be discussed later, $\Lambda$ is in fact an element of $S O(d, d \mid \mathrm{Z})$. This is the subgroup with determinant one and integer valued entries in the basis where the metric is given by (2.45). The Weyl spinor representations of $S O(d, d \mid \mathrm{Z})$ are also integral, that is the representation matrices have integer entries. This statement, which is implicit in papers discussing Tduality of Type II string theory, will be proven in the Appendix A. Since the spinor representation of $S O(d, d \mid \mathbf{Z})$ will be used extensively in the following sections, recall that the vector and spinor representations are related by

$$
\mathcal{S}^{-1} \gamma_{s} \mathcal{S}=\Lambda_{s}^{p} \gamma_{p}
$$

and the gamma matrices satisfy

$$
\left\{\gamma_{s}, \gamma_{p}\right\}=2 J_{s p}
$$

Finally, one can show by direct calculation that the commutation relations of the constant curvature connection and $Z_{i}$ have the form

$$
\left[\nabla^{i}, Z_{j}\right]=i H_{j}^{i} Z_{j}
$$

where there is no sum over $j$ and $H=(\tilde{n}-\widetilde{M} \Theta)^{-1} N$. Note that $H$ can also be written in terms of $\Theta$ and some of the block components of $\Lambda$

$$
H^{-1}=\mathcal{C} \Theta+\mathcal{D}
$$

Finally, the following identities will be useful in later sections

$$
H=\tilde{n}^{-1} Q^{2} N
$$




$$
\begin{aligned}
\operatorname{det} H & =(q \operatorname{det}(Q) / n)^{2}, \\
\operatorname{det}\left(Q^{2}\right) & =\left(1-\frac{\operatorname{tr}(\widetilde{M} \Theta)}{2 \tilde{n}}\right)^{-2} . \\
M & =2 \pi n Q^{-1} \mathcal{F}_{(0)} Q^{-T}, \\
M^{i j} \varepsilon_{i j l} & =M^{i j} \varepsilon_{i j k} Q_{l}^{k} .
\end{aligned}
$$

Note that with the exception of the last relation all the others are valid for tori of arbitrary dimension provided one works on the bundles discussed in Section 2.3.

\subsection{Two and Three Dimensional Solutions}

Although the twisted two dimensional case has been discussed extensively in the literature $[24,7,39,52]$, I will review it here in a form that readily admits generalization to higher dimensional compactifications. I will then give a complete description of the three dimensional adjoint bundles.

In the two dimensional case the antisymmetric matrices $\Theta$ and $M$ have the form

$$
\Theta=\left(\begin{array}{cc}
0 & \theta \\
-\theta & 0
\end{array}\right), \quad M=\left(\begin{array}{cc}
0 & m \\
-m & 0
\end{array}\right)
$$

where $\theta$ is the deformation parameter and $m$ is the magnetic flux, which is interpreted as the number of $\mathrm{D} 2$ branes wrapping the two-torus.

One can verify that the integers

$$
\left(a^{i}\right)=(\tilde{m}, 0), \quad\left(b^{i}\right)=(0,1),
$$

where $n=q \tilde{n}$ and $m=q \tilde{m}$, satisfy (2.30). Then choosing $s_{i}=(0,1)$ and $t_{i}=(b, 0)$, where $b$ is an integer such that $a \tilde{n}-b \tilde{m}=1$, one has $N=I_{2}$. One can now 
use (2.38) and (2.43) to find

$$
\Lambda=\left(\begin{array}{cc}
a I_{2} & b \varepsilon \\
-\tilde{m} \varepsilon & \tilde{n} I_{2}
\end{array}\right)
$$

where $\varepsilon$ is a two dimensional matrix with the only nonvanishing entries given by $\varepsilon_{12}=-\varepsilon_{21}=1$. Group elements of the form above are in an $S L(2, \mathbf{Z})$ subgroup of $S O(2,2 \mid \mathbf{Z})$. This subgroup is isomorphic with one of the Weyl spinor representations of $S O(2,2 \mid \mathbf{Z})$. This feature is not generic for higher dimensional compactifications and reflects the fact that $S O(2,2 \mid \mathbf{Z}) \sim S L(2, \mathbf{Z}) \times S L(2, \mathbf{Z})$, so that it is not simple.

The algebra of the $Z_{i}^{\prime}$ 's is then determined by $\Theta^{\prime}$ which is given by the fractional transformation (2.41). In two dimensions, the $S O(2,2 \mid \mathrm{Z})$ fractional transformation (2.41) can also be written in the more familiar form, used in [24, 39], as a $S L(2, \mathbf{Z})$ fractional transformation acting on $\theta$

$$
\theta^{\prime}=\frac{a \theta+b}{\tilde{m} \theta+\tilde{n}}
$$

One can also check that the other $S L(2, \mathbf{Z})$ subgroup, made of elements of the form

$$
\left(\begin{array}{cc}
R & 0 \\
0 & \left(R^{T}\right)^{-1}
\end{array}\right)
$$

acts trivially on $\theta$. This subgroup is generalized to $S L(d, \mathbf{Z})$ in compactifications on a $d$-dimensional torus, and will play in important role later, but only for the two dimensional compactification it leaves $\Theta$ invariant. The $Z_{i}$ 's then obey the following algebra 


$$
Z_{1} Z_{2}=e^{2 \pi i \theta^{\prime}} Z_{2} Z_{1}
$$

As will be shown shortly, the rank of the gauge group and the magnetic flux transform in an integral Weyl spinor representation of $S O(2,2 \mid \mathbf{Z})$. Using the creation and annihilation operators introduced in the Appendix A one can write such a spinor as

$$
n|0\rangle+m a_{1}^{\dagger} a_{2}^{\dagger}|0\rangle .
$$

Using (2.46) one can show that the spinor representation of (2.51) transforms the above state into $q|0\rangle$. In the Weyl basis one can write the action as

$$
\left(\begin{array}{l}
q \\
0
\end{array}\right)=S\left(\begin{array}{l}
n \\
m
\end{array}\right),
$$

where

$$
S=\left(\begin{array}{cc}
a & -b \\
-\tilde{m} & \tilde{n}
\end{array}\right) .
$$

In Section 3.1 I will show, employing the expansion of the adjoint section in terms of the $Z_{i}$ generators (2.32), how to rewrite the original $U(n)$ NCSYM action on a twisted bundle as a $U(q)$ NCSYM action on a trivial quantum bundle over a torus with deformation parameter $\Theta^{\prime}$. The $S L(2, \mathbf{Z})$ transformation, which relates the deformation parameters and the spinors (2.53) of these two NCSYM, can then be interpreted as a duality transformation inherited from T-duality of Type II string theory. This can be seen as follows. The rank and the bundle of the NCSYM theory determine the $\mathrm{D}$ brane charges in string theory. These charges transform 
in a chiral spinor representation of the target space duality group [86]. Given $n$ and $m$ with greatest common divisor $q$, one can perform a T-duality transformation which takes the original D brane configuration into $q$ D0 branes.

Of course the metric and antisymmetric tensor also transform under this duality, and in the proper limit, which I will explain in detail later, the antisymmetric tensor $B$ transforms separately by fractional transformation just as in (2.41). Since the parameters $\Theta_{i j}$ of the NCSYM theory are identified with $B_{i j}$, the background expectation value of the NS antisymmetric tensor of the compactified auxiliary string theory, the expected transformation under target space dualities is (2.41).

Next we turn to the three dimensional case which will be solved by first performing an $S L(3, \mathbf{Z})$ transformation $R$ to bring $M$ in canonical form ${ }^{3}$

$$
M=R M^{0} R^{T}
$$

where

$$
M^{0}=\left(\begin{array}{ccc}
0 & 0 & 0 \\
0 & 0 & m \\
0 & -m & 0
\end{array}\right) .
$$

While it is always possible to find such a transformation, (2.55) does not define it uniquely. I will first find the solution corresponding to $M^{0}$, and then obtain the general solution by using such an $R$.

First note that $M^{0}$ corresponds to a background magnetic field with flux only through the (23) plane, which suggests that the solution should closely resemble

\footnotetext{
${ }^{3}$ It is always possible to bring an antisymmetric matrix in canonical form using $S L(3, \mathbf{R})$ but here one has to do this using an integral matrix.
} 
the two dimensional one. As before,

$$
\left(a_{0}^{i}\right)=(0, \tilde{m}, 0),\left(b_{0}^{i}\right)=(0,0,1)
$$

satisfy (2.30). Similarly if one sets

$$
\left(s_{i}^{0}\right)=(0,0,1),\left(t_{i}^{0}\right)=(0, b, 0),
$$

one can satisfy (2.37) with the $N^{0}$ matrix given by

$$
N^{0}=\left(\begin{array}{ccc}
\tilde{n} & 0 & 0 \\
0 & 1 & 0 \\
0 & 0 & 1
\end{array}\right)
$$

The diagonal entries of $N^{0}$ divided by $\tilde{n}$ have the interpretation of wave numbers. Thus one can see that twisting the boundary conditions allows for fractional wave numbers in the second and third directions. Using (2.57) one finds

$$
L^{0}=\left(\begin{array}{ccc}
0 & 0 & 0 \\
0 & 0 & -b \\
0 & b & 0
\end{array}\right)
$$

One can now use (2.43) to find the $S O(3,3 \mid \mathbf{Z})$ matrix

$$
\Lambda^{0}=\left(\begin{array}{cccccc}
1 & 0 & 0 & 0 & 0 & 0 \\
0 & a & 0 & 0 & 0 & b \\
0 & 0 & a & 0 & -b & 0 \\
0 & 0 & 0 & 1 & 0 & 0 \\
0 & 0 & -\tilde{m} & 0 & \tilde{n} & 0 \\
0 & \tilde{m} & 0 & 0 & 0 & \tilde{n}
\end{array}\right)
$$

Everything so far is just as in the two dimensional case. Note however that in general $\Theta$ will not be in canonical form, that is, it will not have a form similar to $(2.56)$. 
Now, write the general solution for an arbitrary $M$ as

$$
\begin{gathered}
a^{i}=R_{j}^{i} a_{0}^{j}, \quad b^{i}=R_{j}^{i} b_{0}^{j}, \\
s_{i}=s_{j}^{0}, \quad t_{i}=t_{j}^{0} \\
N=R N^{0} \\
\Lambda=\Lambda^{0}\left(\begin{array}{cc}
R^{T} & 0 \\
0 & R^{-1}
\end{array}\right) .
\end{gathered}
$$

Just as in the two dimensional case one finds, using (2.46), the Weyl spinor representation matrices corresponding to (2.58) and (2.59)

$$
\begin{gathered}
S^{0}=\left(\begin{array}{cccc}
a & -b & 0 & 0 \\
-\tilde{m} & \tilde{n} & 0 & 0 \\
0 & 0 & 1 & 0 \\
0 & 0 & 0 & 1
\end{array}\right), \\
S=S^{0}\left(\begin{array}{cc}
1 & 0 \\
0 & R^{T}
\end{array}\right)
\end{gathered}
$$

The rank of the group and the magnetic flux matrix $M$ define a state in the Weyl spinor Fock space

$$
n|0\rangle+\frac{1}{2} M^{i j} a_{i}^{\dagger} a_{j}^{\dagger}|0\rangle
$$

Now one can check that $S$ acts on this spinor as

$$
\left(\begin{array}{l}
q \\
0 \\
0 \\
0
\end{array}\right)=S\left(\begin{array}{c}
n \\
M^{23} \\
M^{31} \\
M^{12}
\end{array}\right) .
$$


It will be convenient to denote the components of the spinor as

$$
\eta=\left(\begin{array}{c}
n \\
M^{23} \\
M^{31} \\
M^{12}
\end{array}\right) .
$$

As will be shown later $(2.60)$ can be used to relate the original theory to a $U(q)$ theory on a trivial bundle. In Appendix A I will show that the Weyl spinor representation of $S O(3,3 \mid \mathbf{Z})$ is in fact isomorphic to $S L(4, \mathbf{Z})$. In this case, in the auxiliary Type IIA string theory, the D0 and D2 branes form $q$ bound states, and the transformation above corresponds to a T-duality transformation that maps the original $\mathrm{D}$ brane configuration into a $q \mathrm{D} 0$ branes.

\subsection{Noncommutative Super Yang-Mills Action}

After discussing how to perform integration on a noncommutative torus one will be ready to write the noncommutative Super Yang-Mills action. In the classical case the integral is a linear map that associates to a function its zero mode Fourier coefficient. Similarly for an element of $\mathcal{A}_{\Theta}$ of the form $a=\sum a_{i_{1} i_{2} \ldots i_{d}} U_{1}^{i_{1}} U_{2}^{i_{2}} \ldots U_{d}^{i_{d}}$ define the integral as

$$
\int d^{d} \sigma a \stackrel{\text { def }}{=}(2 \pi)^{d} a_{00 \ldots 0}
$$

One can check that this definition has all the desirable properties of the classical integral, such as linearity and translation invariance in $\sigma_{i}$. For definiteness, in the remainder of this section I will discuss the three dimensional case. 
When twisted $U(n)$ theories are considered, it was found in $[24,39]$ that the integral must be normalized in a particular way to find a duality invariant spectrum. The normalization can also be obtained directly as the Jacobian of a change of integration variables. Note that the integrand, which is the trace of an adjoint section, obeys the following periodicity

$$
\operatorname{tr} \Psi\left(\sigma_{i}\right)=\operatorname{tr} \Psi\left(\sigma_{i}+2 \pi\left(Q^{-1}\right)_{i}^{j}\right)
$$

Since $\operatorname{tr} \Psi\left(\sigma_{i}\right)$ does not have periodicity $2 \pi$ in $\sigma_{i}$ it can not be expanded in terms of the $U_{i}$ variables. One can define new variables $\widehat{\sigma}_{i}=\sigma_{j} Q_{k}^{j} R_{i}^{k}$ and $\widehat{U}_{i}=e^{\widehat{\sigma}_{i}}$, where $R$ is an arbitrary $S L(3, \mathbf{Z})$ transformation. In the following sections I will take $R$ to be the matrix that brings $M$ into canonical form (2.55). Then

$$
\int d^{3} \sigma \operatorname{tr} \Psi(\sigma)=\int d^{3} \widehat{\sigma}\left|\operatorname{det}\left(Q^{-1}\right)\right| \operatorname{tr} \Psi\left(\widehat{\sigma} Q^{-1}\right)
$$

where $\operatorname{det}\left(Q^{-1}\right)$ is the Jacobian of the coordinate transformation, and the second integral can now be performed as discussed above, since the integrand has an expansion in terms of the $\widehat{U}_{i}$ variables. Using the expansion (2.32) of $\Psi$ one obtains

$$
\int d^{3} \sigma \operatorname{tr} \Psi(\sigma)=(2 \pi)^{3} \tilde{n}\left|\operatorname{det}\left(Q^{-1}\right)\right| \sum_{a=1}^{q} \Psi_{000}^{a a} .
$$

The Super Yang-Mills action on a noncommutative three-torus is given by

$$
\begin{aligned}
\mathcal{S}^{U(n)}= & \frac{1}{g_{S Y M}^{2}} \int d t \int d^{3} \sigma \sqrt{\operatorname{det}\left(G^{i j}\right)} \operatorname{tr}\left(\frac{1}{2} G_{i j} \mathcal{F}^{0 i} \mathcal{F}^{0 j}-\right. \\
& \frac{1}{4} G_{i j} G_{k l}\left(\mathcal{F}^{i k}-\mathcal{F}_{(0)}^{i k}\right)\left(\mathcal{F}^{j l}-\mathcal{F}_{(0)}^{j l}\right)+ \\
& \frac{1}{2} \sum_{a} \dot{X}^{a} \dot{X}^{a}-\frac{1}{2} \sum_{a} G_{i j}\left[D^{i}, X^{a}\right]\left[D^{j}, X^{a}\right]+
\end{aligned}
$$




$$
\left.\frac{1}{4} \sum_{a, b}\left[X^{a}, X^{b}\right]\left[X^{a}, X^{b}\right]+\text { fermions }\right),
$$

where $\mathcal{F}^{i j}=i\left[D^{i}, D^{j}\right]$ and $\mathcal{F}_{0}^{i j}=i\left[\nabla^{i}, \nabla^{j}\right]$. I have subtracted the constant part of the field strength in the second line of equation (2.63). This is equivalent to adding a constant to the Lagrangian, or equivalently to the Hamiltonian, and has the effect of setting the vacuum energy to zero. The noncommutative pure gauge theory action was written first in [22] and the maximally supersymmetric $U(n)$ NCSYM gauge theory action was written in $[24,68]$.

For the compactification of the auxiliary Type IIA string theory without wrapped D2 branes, the above action can be obtained directly from the Matrix action. One has to show that the trace over infinite dimensional matrices reduces to a finite dimensional trace and an integral. A formal argument for the commutative case was given in [78] and discussed in detail in [79]. The same argument extends to the noncommutative case. A brief argument was given in [24] showing how to extend this construction when there are D2 branes wrapped on the torus in the auxiliary Type IIA string theory, corresponding to magnetic fluxes in the NCSYM gauge theory. Here I will just make the assumption that the NCSYM action is independent of the D2 brane charges and that adding D2 branes only results in changing the quantum adjoint bundle. I will provide evidence for this in Section 3.2 . 


\section{Chapter 3}

\section{Dualities of the Matrix Model from T-Duality of the Type II String}

In Section 3.1 I will start with the $U(n)$ NCSYM action (2.63) on a twisted quantum bundle with magnetic fluxes $M$ and deformation parameter $\Theta$, and show that after a sequence of field redefinitions it can be rewritten as a $U(q)$ NCSYM action on a trivial bundle over a quantum torus with deformation parameter $\Theta^{\prime}$.

Then in Section 3.2 I will take the small $\alpha^{\prime}$ and small compactification volume limit in the auxiliary Type IIA string theory and obtain the transformation properties of the metric, antisymmetric tensor and string coupling constant. These are then compared with the transformation in Section 3.1 using the standard relations between String theory and SYM gauge theory.

Section 3.3 contains the dictionary between the physical language used in these constructions and the more abstract mathematical language of Connes and Rieffel. The last section contains some gauge equivalent formulations closely resembling standard constructions in the commutative case.

\section{1 $S O(3,3 \mid \mathbf{Z})$ Duality of Super Yang-Mills}


Using the matrix $H$ defined in (2.48) one can make the following constant curvature and field redefinitions

$$
\begin{gathered}
\widehat{\nabla}^{i} \stackrel{\text { def }}{=}\left(H^{-1}\right)_{j}^{i} \nabla^{j}, \widehat{A}^{i} \stackrel{\text { def }}{=}\left(H^{-1}\right)_{j}^{i} A^{j}, \\
\widehat{D}^{i} \stackrel{\text { def }}{=}\left(H^{-1}\right)_{j}^{i} D^{j}, \\
\widehat{\mathcal{F}}^{k l}=\left[\widehat{\nabla}^{k}, \hat{A}^{l}\right]-\left[\widehat{\nabla}^{l}, \widehat{A}^{k}\right]-i\left[\widehat{A}^{k}, \widehat{A}^{l}\right] .
\end{gathered}
$$

In terms of the new variables the commutator of the constant curvature connection and the $Z_{i}$ 's takes the simple form,

$$
\left[\widehat{\nabla}^{i}, Z_{j}\right]=i \delta_{j}^{i} Z_{j},
$$

and the curvature can be expressed as

$$
\mathcal{F}^{i j}=\mathcal{F}_{(0)}^{i j}+H_{k}^{i} H_{l}^{j} \widehat{\mathcal{F}}^{k l} .
$$

One can now rewrite the action in terms of the hatted variables and perform the change of coordinates (2.62)

$$
\begin{gathered}
\mathcal{S}^{U(n)=} \frac{1}{g^{\prime 2}{ }_{S Y M}} \int d t \int d^{3} \widehat{\sigma} \sqrt{\operatorname{det}\left(G^{\prime i j}\right)} \frac{1}{\tilde{n}} \operatorname{tr}\left(\frac{1}{2} G_{i j}^{\prime} \widehat{\mathcal{F}}^{0 i} \widehat{\mathcal{F}}^{0 j}-\right. \\
\frac{1}{4} G_{i j}^{\prime} G_{k l}^{\prime} \widehat{\mathcal{F}}^{i k} \widehat{\mathcal{F}}^{j l}+ \\
\frac{1}{2} \sum_{a} \dot{X}^{a} \dot{X}^{a}-\frac{1}{2} \sum_{a} G_{i j}^{\prime}\left[\widehat{D}^{i}, X^{a}\right]\left[\widehat{D}^{j}, X^{a}\right]+ \\
\left.\frac{1}{4} \sum_{a, b}\left[X^{a}, X^{b}\right]\left[X^{a}, X^{b}\right]+\text { fermions }\right) .
\end{gathered}
$$

I have introduced a new gauge coupling and metric given by

$$
g_{S Y M}^{\prime 2}=\tilde{n}\left|\operatorname{det}\left(Q^{-1}\right)\right| g_{S Y M}^{2}
$$




$$
G^{i j}=\left(H^{-1}\right)_{k}^{i}\left(H^{-1}\right)_{l}^{j} G^{k l}
$$

and used (2.50) to make these substitutions.

Next I will introduce primed variables $\sigma_{i}^{\prime}, U_{i}^{\prime}$ and partial derivatives $\partial^{\prime i}$ satisfying

$$
\begin{gathered}
{\left[\sigma_{i}^{\prime}, \sigma_{j}^{\prime}\right]=-2 \pi i \Theta_{i j}^{\prime},} \\
{\left[\partial^{\prime i}, \sigma_{j}^{\prime}\right]=\delta_{j}^{i}, \quad\left[\partial^{\prime i}, \partial^{\prime j}\right]=0,} \\
U_{i}^{\prime} \stackrel{\text { def }}{=} e^{i \sigma_{i}^{\prime}} \\
U_{i}^{\prime} U_{j}^{\prime}=e^{2 \pi i \Theta_{i j}^{\prime}} U_{j}^{\prime} U_{j}^{\prime} .
\end{gathered}
$$

Comparing the algebra satisfied by $Z_{i}$ and $\widehat{\nabla}^{i}$ on one hand and $U_{i}^{\prime}$ and $\partial_{i}^{\prime}$ on the other, one can see that all the commutation relations are the same except that the $\widehat{\nabla}^{i}$ 's do not commute while the $\partial^{\prime i}$ 's do. The dynamical variables of the theory are the $c$-number coefficients appearing in the expansion (2.32) of the adjoint sections in terms of $Z_{i}$ 's. Since in the action, the constant curvature covariant derivatives only appear in commutators with the $Z_{i}$ 's and not with each other, substituting $U_{i}^{\prime}$ and $\partial^{\prime i}$ for $Z_{i}$ and $\widehat{\nabla}^{i}$ leaves the dynamics invariant. A similar construction was also considered in [68]. The integral and trace of the $U(n)$ theory can be translated to a $U(q)$ integral using the definition of the integral (2.61)

$$
\int d^{3} \widehat{\sigma} \frac{1}{\tilde{n}} \operatorname{tr} \Psi\left(Z_{i}\right)=\int d^{3} \sigma^{\prime} \operatorname{tr}_{q} \Psi\left(U_{i}^{\prime}\right)=(2 \pi)^{3} \sum_{a=1}^{q} \Psi_{000}^{a a}
$$

Making these substitutions one obtains the $U(q)$ action

$$
\mathcal{S}^{U(q)}=\frac{1}{g_{S Y M}^{\prime 2}} \int d t \int d^{3} \sigma^{\prime} \sqrt{\operatorname{det}\left(G^{\prime i j}\right)} \operatorname{tr}_{q}\left(\frac{1}{2} G_{i j}^{\prime} \mathcal{F}^{\prime 0 i} \mathcal{F}^{\prime 0 j}\right.
$$




$$
\begin{gathered}
\frac{1}{4} G_{i j}^{\prime} G_{k l}^{\prime} \mathcal{F}^{i k} \mathcal{F}^{\prime j l}+ \\
\frac{1}{2} \sum_{a} \dot{X}^{a} \dot{X}^{a}-\frac{1}{2} \sum_{a} G_{i j}^{\prime}\left[D^{\prime i}, X^{a}\right]\left[D^{\prime j}, X^{a}\right]+ \\
\left.\frac{1}{4} \sum_{a, b}\left[X^{a}, X^{b}\right]\left[X^{a}, X^{b}\right]+\text { fermions }\right)
\end{gathered}
$$

where

$$
D^{\prime i} \stackrel{\text { def }}{=} \partial^{\prime i}-i A^{i i}, \mathcal{F}^{\prime i j}=i\left[D^{\prime i}, D^{\prime j}\right]
$$

are the $U(q)$ connection and curvature. This shows that the original $U(n)$ theory is equivalent to a $U(q)$ NCSYM theory with gauge coupling given by (3.1) and defined on a trivial adjoint bundle over a noncommutative torus with deformation parameter $\Theta^{\prime}$ and metric given by (3.2).

In general two NCSYM theories are dual to each other if there exists an element $\Lambda$ of $S O(3,3 \mid \mathbf{Z})$ with Weyl spinor representation matrix $S$, such that their defining parameters are related as follows

$$
\begin{gathered}
\bar{\Theta}=(\mathcal{A} \Theta+\mathcal{B})(\mathcal{C} \Theta+\mathcal{D})^{-1}, \\
\left(\begin{array}{c}
\bar{n} \\
\bar{M}^{23} \\
\bar{M}^{31} \\
\bar{M}^{12}
\end{array}\right)=S\left(\begin{array}{c}
n \\
M^{23} \\
M^{31} \\
M^{12}
\end{array}\right), \\
\bar{G}^{i j}=(\mathcal{C} \Theta+\mathcal{D})_{k}^{i}(\mathcal{C} \Theta+\mathcal{D})_{l}^{j} G^{k l}, \\
\bar{g}_{S Y M}^{2}=\sqrt{|\operatorname{det}(\mathcal{C} \Theta+\mathcal{D})|} g_{S Y M}^{2},
\end{gathered}
$$

where I used (2.49) in the last two equations. While $\Theta$ in (3.3) and the rank and magnetic flux numbers in (3.4) transform separately and the duality group action 
can be seen explicitly, the transformation of the gauge coupling and the metric also depends on $\Theta$. Note that $\mathcal{C} \Theta+\mathcal{D}$ satisfies a group property. If $\Lambda_{3}=\Lambda_{2} \Lambda_{1}$ and $\Theta^{\prime}=\Lambda_{1}(\Theta)$ then

$$
\mathcal{C}_{3} \Theta+\mathcal{D}_{3}=\left(\mathcal{C}_{2} \Theta^{\prime}+\mathcal{D}_{2}\right)\left(\mathcal{C}_{1} \Theta+\mathcal{D}_{1}\right)
$$

For a nonvanishing $\bar{n}$, the sign ambiguity that exists when one tries to associate to a $S O(3,3 \mid \mathbf{Z})$ transformation its spinor representation matrix, can be removed by requiring that $\bar{n}$ is positive. Strictly speaking, one should not consider duality transformations for which $\bar{n}$ vanishes since in this case the description in terms of gauge theories becomes singular. 


\section{$3.2 \quad$ Target Space Duality}

Next I will show that the $S O(3,3 \mid \mathbf{Z})$ duality discussed in the previous section is the realization in NCSYM gauge theories of T-duality in the auxiliary Type IIA string theory. This relation is described by the following diagram.

\begin{tabular}{|c|c|c|c|c|}
\hline IIA & $\begin{array}{c}n, \quad M^{i j} \\
g_{s} \\
G_{i j} \\
B_{i j}\end{array}$ & $\longleftrightarrow$ & NCSYM & $\begin{array}{c}U(n), M^{i j} \\
g_{S Y M} \\
G^{i j} \\
\Theta_{i j}=B_{i j}\end{array}$ \\
\hline & $I$ & & \multicolumn{2}{|r|}{$I$} \\
\hline IIA & $\begin{array}{c}q, \quad M^{i j j}=0 \\
g_{s}^{\prime} \\
G_{i j}^{\prime} \\
B_{i j}^{\prime}\end{array}$ & $\longleftrightarrow$ & NCSYM & $\begin{array}{c}U(q), M^{\prime i j}=0 \\
g_{S Y M}^{\prime} \\
G^{\prime i j} \\
\Theta_{i j}^{\prime}=B_{i j}^{\prime}\end{array}$ \\
\hline
\end{tabular}

The right side of the diagram shows the equivalence described in Section (3.1). The horizontal arrows represent the Connes, Douglas and Schwarz conjecture [24]. The left side of the diagram contains the string coupling, D brane charges, and compactification moduli of the two auxiliary Type IIA string theories corresponding to the NCSYM's on the right. The additional moduli corresponding to Ramond-Ramond backgrounds are beeing set to zero in this chapter and will be considered separately in Chapterrefchap4. Note that the NCSYM metric is the inverse of the Type IIA metric as indicated by the index position, the deformation 
parameter equals the NS antisymmetric tensor, and the rank and magnetic flux numbers translate into D0 brane number and D2 brane winding. Finally the SYM and string coupling are related by (2.5).

For compactification on $T^{2}$, the duality transformation (2.54) can be written as an $S L(2, \mathbf{Z})$ mapping class group transformation conjugated by a T-duality in the $x^{1}$ direction ${ }^{1}$. The sequence of duality transformations is shown in figure 3.1 . Under T-duality in the $x^{1}$-direction the two D0-branes are mapped into D1-strings wrapping the horizontal cycle and the D2-brane into a D1 string wrapping the oblique cycle. The T-dual torus is not rectangular for a nonvanishing $\theta$. In fact the D1-strings can be in a lower energy state obtained by minimizing their total length. This corresponds to the fact that the original D0 and D2-branes form a bound state. Under the $S L(2, \mathrm{Z})$ mapping class group transformation we can arrange the D1-string along the horizontal axis of the torus. This is just a relabeling of the defining 1-cycle of the torus. Finally after another T-duality in the $x^{1}$-direction we arrive at the final configuration which contains a single D0-branes and the final Ramond-Ramond modulus given by $\theta^{\prime}$.

In the remainder of this section I will calculate the relation between the parameters of the two auxiliary Type IIA string theories. First I will describe how the metric, antisymmetric tensor and the string coupling transform under an arbitrary T-duality transformation, and then take the limit

$$
\alpha^{\prime} \rightarrow 0, \quad G_{i j} \rightarrow 0
$$

\footnotetext{
${ }^{1}$ The T-duality in the $x^{1}$-direction is an element of $O(2,2 \mid \mathbf{Z})$ of negative determinant.
} 


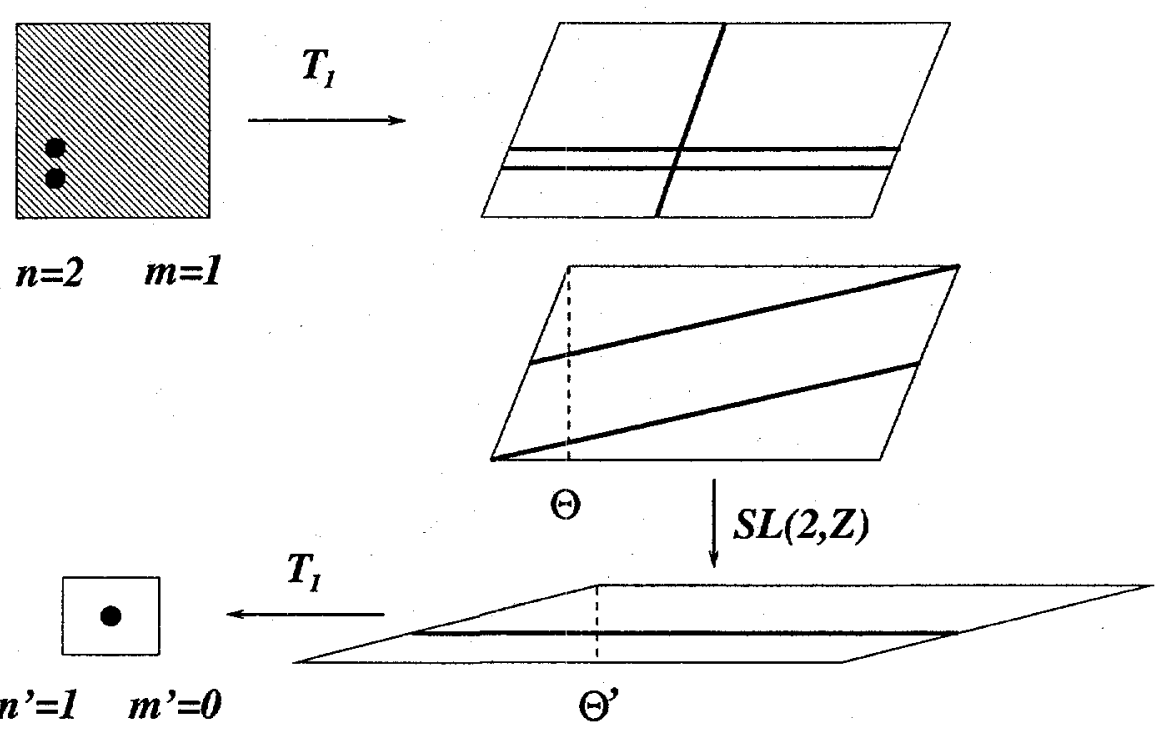

Figure 3.1: The covering space of $T^{2}$ with one D0-brane per cell

keeping $\alpha^{\prime-2} G_{i j}$ constant. This is the limit proposed by Seiberg and Sen [69, 73] and briefly discussed in the introduction. However, in this limit the auxiliary Type IIA string metric vanishes. Instead I will calculate directly the inverse metric of the NCSYM theory which, after including factors of $\alpha^{\prime}$, is given by $\alpha^{\prime-2} G_{i j}$.

Under the T-duality group $S O(d, d \mid \mathrm{Z})$ the metric and NS antisymmetric tensor $^{2}$ transform together by fractional transformations [35]

$$
G^{\prime}+B^{\prime}=(\mathcal{A}(G+B)+\mathcal{B})(\mathcal{C}(G+B)+\mathcal{D})^{-1}
$$

Using the identification between $\Theta$ and $B$ one obtains $H^{-1}=\mathcal{C} B+\mathcal{D}$. Then, after some matrix algebra, one can write the symmetric and antisymmetric part

\footnotetext{
${ }^{2}$ Hopefully there is no confusion between $B$, denoting the NS tensor, and $\mathcal{B}$ which is the upper
} right block of $\Lambda$. 
of $(3.9)$ as

$$
\begin{aligned}
& G^{\prime}=H^{T} G\left(1-(H \mathcal{C} G)^{2}\right)^{-1} H \\
& B^{\prime}=(\mathcal{A} B+\mathcal{B})(\mathcal{C} B+\mathcal{D})^{-1}-H^{T} G H \mathcal{C} G\left(1-(H \mathcal{C} G)^{2}\right)^{-1} H
\end{aligned}
$$

One derives this using the fact that $H \mathcal{C}$ is antisymmetric. This can be shown using

$$
(\mathcal{C} B+\mathcal{D})^{-1}=\left(\mathcal{A}-(\mathcal{A} B+\mathcal{B})(\mathcal{C} B+\mathcal{D})^{-1} \mathcal{C}\right)^{T}
$$

which follows from (2.44). Note that (3.10) and (3.11) have simple expansions in $G$. For an elementary T-duality in the $x^{1}$ direction the string coupling constant transforms as

$$
g_{S}^{\prime}=g_{S} G_{11}^{-1 / 2}
$$

Taking the limit (3.8) in (3.11) one can see that the antisymmetric tensor itself transforms by fractional transformation ${ }^{3}$

$$
B^{\prime}=(\mathcal{A B}+\mathcal{B})(\mathcal{C} B+\mathcal{D})^{-1}
$$

To find the duality transformation of the metric, I will reinstate factors of $\alpha^{\prime}$ in (3.10) since the $S O(3,3 \mid \mathrm{Z})$ transformations are defined to act on dimensionless fields. Now, take the limit (3.8) and to first order in the dimensionless metric $\alpha^{\prime-1} G_{i j}$ one has

$$
\alpha^{\prime-2} G^{\prime}=(\mathcal{C} B+\mathcal{D})^{-T}\left(\alpha^{\prime-2} G\right)(\mathcal{C} B+\mathcal{D})^{-1}
$$

\footnotetext{
${ }^{3}$ This is consistent with the fact that the action by fractional transformations preserves the antisymmetry of the matrices.
} 
Making the identification $B=\Theta$, one recognizes above the $H$ matrix defined in (2.49).

Finally using (3.12), one can also calculate how the string coupling transforms under duality. It was shown in [67] that the $S O(d, d \mid \mathbf{Z})$ group is generated by a set of simple elements. These are written explicitly in the Appendix A. For each of these generators one can check using (3.12) that the string coupling transforms as

$$
g_{S}^{\prime}=g_{S}|\operatorname{det}(\mathcal{C} B+\mathcal{D})|^{-1 / 2} .
$$

In fact (3.15) is true for an arbitrary transformation because $\mathcal{C} B+\mathcal{D}$ satisfies the group property (3.7).

Comparing the T-duality relations (3.13), (3.14) and (3.15) with the NCSYM duality relations (3.3), (3.5) and (3.6), using (2.5) to relate the string and gauge couplings, one sees that indeed the two dualities coincide.

\subsection{Projective Modules and Morita Equivalence}

A quantum vector bundle is a projective $\mathcal{A}$-module $\mathcal{E}$. First consider the classical commutative picture. The set $\mathcal{E}$ of global sections of a vector bundle over a base space $X$ has the structure of a projective module over the algebra $C(X)$. Having a module essentially means that one can add sections and can multiply them by functions. Not all modules over a commutative algebra are vector bundles. For example the set of sections on a space consisting of a collection of fibers 
of different dimensions over a base space also form a module. However, projective modules over the algebra of functions on a topological space are in one to one correspondence with vector bundles over that space. By definition a projective module is a direct summand in a free module. A free module $\mathcal{E}_{0}$ over an algebra $\mathcal{A}$ is a module isomorphic to a direct sum of a finite number of copies of the algebra

$$
\mathcal{E}_{0}=\mathcal{A} \oplus \ldots \oplus \mathcal{A}
$$

Trivial bundles correspond to free modules since the description of their sections in terms of components is global, and each component is an element of $C(X)$. For every vector bundle one can find another one such that their direct sum is a trivial bundle. In dual language this implies that the module of sections $\mathcal{E}$ is projective

$$
\mathcal{E}_{0}=\mathcal{E} \oplus \mathcal{E}^{\prime}
$$

Again it is nontrivial to show the converse, that every projective module is isomorphic to the set of sections of some vector bundle. Finally projective modules over noncommutative algebras are the quantum version of vector bundles.

In the noncommutative case one distinguishes between left and right projective modules. Multiplying fundamental sections from the right with elements of $\mathcal{A}_{\Theta}$ preserves the boundary conditions (2.15) while multiplication on the left gives something that no longer is a global section. Thus the set of sections of the fundamental bundle form a right projective module over the $\mathcal{A}_{\Theta}$ algebra which I will denote $\mathcal{F}_{\eta}^{\ominus}$. This is no longer true for the adjoint sections since in (2.16) the transition functions multiply from both the left and right. However one can check 
that the adjoint are both left and right projective modules over the $\mathcal{A}_{(-\Theta)}$ algebra. Also the fundamental is a left projective modules over the $\mathcal{A}_{(-\Theta)}$ algebra. This is because the exponents of the $\mathcal{U}_{i}$ 's satisfy

$$
\left[i \sigma_{i}-2 \pi \Theta_{i j} \partial^{j}, \sigma_{k}\right]=0
$$

thus the $\mathcal{U}_{i}$ 's can be commuted over the transition functions in (2.15) and (2.16). Additionally, the fact that $\mathcal{F}_{n, m}^{\Theta}$ is both a left $\mathcal{A}_{(-\Theta)}$-module and a right $\mathcal{A}_{\Theta}$-module can be understood as follows. Since $\left[\mathcal{U}_{i}, \sigma_{j}\right]=0$ one also has

$$
\mathcal{U}_{i} \Phi\left(\sigma_{i}\right)=\Phi\left(\sigma_{i}\right) U_{i}
$$

where I dropped the derivatives when there was nothing to their right. Let $a$ be an element of $\mathcal{A}_{(-\Theta)}$

$$
a=\sum_{i_{1} \ldots i_{d} \in \mathbf{Z}} a_{i_{1} \ldots i_{d}} \mathcal{U}_{1}^{i_{1}} \ldots \mathcal{U}_{d}^{i_{d}}
$$

Thus multiplying on the left with $a$ is equivalent to multiplying on the right with $\tilde{a}$

$$
a \Phi=\Phi \tilde{a},
$$

where $\tilde{a}=\sum_{i_{1} \ldots i_{d} \in \mathbf{Z}} a_{i_{1} \ldots i_{d}} U_{d}^{i_{d}} \ldots U_{1}^{i_{1}}$ is the same function as $a$ but with $\mathcal{U}_{i}$ 's as arguments and with all the factors written in reversed order.

In the remaining of this chapter I will only consider the two dimensional case and use the gauge (2.14). The construction in Chapter 2 is equivalent to the projective modules discussed in [24]. By solving the boundary conditions one goes from a local to a global description. Here I will present explicit formulae for this 
equivalence. First one has to express the left actions on the fundamental sections as actions on the Hilbert space [39]. For example the action of the $Z_{i}$ generators is given by

$$
\left(Z_{1} \widehat{\phi}\right)_{j}(x)=\widehat{\phi}_{j-a}\left(x-\frac{1}{m}\right), \quad\left(Z_{2} \widehat{\phi}\right)_{j}(x)=e^{-2 \pi i j / m} e^{2 \pi i x /(n+m \theta)} \widehat{\phi}_{j}(x)
$$

This can be written as

$$
Z_{1}=W_{1}^{a} V_{1}, \quad Z_{2}=W_{2} V_{2}
$$

where $V_{i}$ and $W_{i}$ are operators acting on the Hilbert space as

$$
\begin{gathered}
\left(V_{1} \widehat{\phi}\right)_{j}(x)=\widehat{\phi}_{j}\left(x-\frac{1}{m}\right), \quad\left(V_{2} \widehat{\phi}\right)_{j}(x)=e^{2 \pi i x /(n+m \theta)} \widehat{\phi}_{j}(x) \\
\left(W_{1} \widehat{\phi}\right)_{j}(x)=\widehat{\phi}_{j-1}(x), \quad\left(W_{2} \widehat{\phi}\right)_{j}(x)=e^{-2 \pi i j / m} \widehat{\phi}_{j}(x)
\end{gathered}
$$

These operators satisfy the following relations

$$
V_{1} V_{2}=e^{-2 \pi i /[m(n+m \theta)]} V_{2} V_{1}, \quad W_{1} W_{2}=e^{2 \pi i / m} W_{2} W_{1}, \quad\left[V_{i}, W_{j}\right]=0
$$

and can be used to express other operators acting in the Hilbert space. For example $\mathcal{U}_{1}=W_{1} V_{1}^{n+m \theta}$ and $\mathcal{U}_{2}=W_{2}^{n} V_{2}^{n+m \theta}$.

Now I will present the correspondence between [24] and the approach followed here ${ }^{4}$. The two integers $p$ and $q$ and the angular variable $\theta_{\mathrm{CDS}}$ labeling the projective module $\mathcal{H}_{p, q}^{\theta_{\mathrm{CDS}}}$ of [24], and $\theta_{\mathrm{CDS}}^{\prime}$ can be expressed in terms of the quantities used here

$$
p=n, \quad q=-m, \quad \theta_{\mathrm{CDS}}=\Theta_{12}, \quad \theta_{\mathrm{CDS}}^{\prime}=\Theta_{12}^{\prime} .
$$

\footnotetext{
${ }^{4}$ We follow here the same notation as in $[39,52]$ except for an overall minus sign in the definition of $\theta$.
} 
Then $\mathcal{F}_{n, m}^{\Theta} \cong \mathcal{H}_{n,-m}^{\Theta_{12}}$. The Hilbert space representation of [24] written in terms of the function $f(s, k)$ with $s \in \mathbf{R}$ and $k \in \mathbf{Z}_{q}$ is linearly related to the $\widehat{\phi}_{k}(x)$ representation

$$
\widehat{\phi}_{k}(x)=\sum_{l=1}^{m} \mathcal{K}_{k l}\left(\mathcal{S}_{\left(-\frac{m}{n+m \theta}\right)} f\right)(x, l) .
$$

Here $\mathcal{K}$ is an $m \times m$ representation changing matrix defined as in (2.20) but for $m$-dimensional 't Hooft matrices, and $\mathcal{S}_{\lambda}$ is the rescaling operator $\left(\mathcal{S}_{\lambda} f\right)(x, k)=$ $f(\lambda x, k)$ which can be expressed using the ordered exponential

$$
\mathcal{S}_{\lambda}=\lambda E\left((\lambda-1) x, \partial_{x}\right)
$$

Also, using lower case to distinguish them from our current notation which follows [39], the operators in [24] represented in the $\hat{\phi}_{k}(x)$ basis are given by

$$
\begin{gathered}
v_{0}=V_{2}^{n+m \theta}, \quad v_{1}=V_{1}^{n+m \theta}, \quad w_{0}=e^{2 \pi i n / m} W_{2}^{n}, \quad w_{1}=e^{2 \pi i / m} W_{1} \\
z_{0}=e^{2 \pi i / m} Z_{2}, \quad z_{1}=e^{-2 \pi i a / m} Z_{1}^{-1}, \quad u_{0}=e^{2 \pi i n / m} \mathcal{U}_{2}, \quad u_{1}=e^{2 \pi i / m} \mathcal{U}_{1} .
\end{gathered}
$$

Next I will introduce the Morita equivalence of two algebras [60, 63, 67, 68], which can be used to describe a subgroup of the T-duality group of the M-theory compactification in the language of noncommutative SYM gauge theory.

Two $C^{*}$-algebras $\mathcal{A}$ and $\mathcal{A}^{\prime}$ are Morita equivalent if there exists a right $\mathcal{A}$ module $\mathcal{E}$ such that the algebra $E n d_{\mathcal{A}} \mathcal{E}$ is isomorphic to $\mathcal{A}^{\prime}$. Here $E n d_{\mathcal{A}} \mathcal{E}$ denotes the set of endomorphisms of the $\mathcal{A}$-module $\mathcal{E}$. It consists of linear maps $T$ on $\mathcal{E}$ where linearity is not only with respect to $c$-numbers but also with respect to right multiplication by elements of $\mathcal{A}$

$$
T(\Phi f)=T(\Phi) f, \quad \Phi \in \mathcal{E}, \quad f \in \mathcal{A} \text {. }
$$


An example of Morita equivalent algebras is $\mathcal{A}_{\Theta}$ and $\mathcal{A}_{\Theta^{\prime}}$. As discussed above, the projective module associated to the quantum fundamental bundle $\mathcal{F}_{n, m}^{\Theta}$ is a right $\mathcal{A}_{\Theta}$-module. One can prove that $E n d_{\mathcal{A}_{\Theta}} \mathcal{F}_{n, m}^{\Theta}$ is isomorphic to $\mathcal{A}_{\Theta^{\prime}}$. Here I will just show that the two algebras have the same generators. Using (3.17) one has $T(\Phi \tilde{a})=T(a \Phi)$ and $T(\Phi) \tilde{a}=a T(\phi)$ and since $T$ is an endomorphism one obtains $T(a \Phi)=a T(\phi)$, which can also be written as $[T, a]=0$. But the $Z_{i}$ 's were found exactly by requiring that they commute with $\mathcal{U}_{i}^{\prime}$ s so $T \in \mathcal{A}_{\Theta^{\prime}}$.

The physical interpretation of Morita equivalence is that a $U(n)$ SYM gauge theory on the twisted bundle with magnetic flux $m$ is equivalent to a $U(1)$ gauge theory on a dual quantum torus $\mathcal{A}_{\Theta^{\prime}}$. This can be seen as a consequence of the discussion following equation (2.13). The gauge field components $A^{i}$, the scalar fields $X^{a}$, and the components of $\Theta$ are not matrix valued, rather they are onedimensional. The final result is a matrix because the $Z_{i}$ 's are matrices. On the other hand, one can ignore the internal structure of the $Z_{i}$ 's and just regard them as the generators of $\mathcal{A}_{\Theta^{\prime}}$, thus allowing us to reinterpret the original theory as a noncommutative $U(1)$ gauge theory on the quantum torus $\mathcal{A}_{\Theta^{\prime}}$.

Since $\Theta$ is a continuous variable, one can interpolate continuously, through noncommutative SYM theories, between two commutative SYM theories with gauge groups of different rank and appropriate magnetic fluxes. This $S L(2, \mathbf{Z})$ duality subgroup has a nice geometric interpretation in the T-dual picture of [27] where it corresponds to a change of basis of the dual torus lattice [21]. 


\subsection{Gauge Transformations}

In this section I will consider a gauge equivalent formulation of the previous results closely following the treatment of Taylor in [79] of the corresponding commutative case. In that paper a gauge transformation was considered so as to change the standard 't Hooft transition function into trivial transition function in the $X^{2}$ direction. When the transition functions are trivial T-duality has the standard form, i.e. the gauge field translates directly into the position of a Dstring on the dual torus. A similar gauge transformation can be performed in the noncommutative case.

First let us consider a general gauge transformation $g\left(\sigma_{1}, \sigma_{2}\right)$. Just as in the classical case the covariant derivatives transform as $D_{i}^{\prime}=g^{-1} D_{i} g$ resulting in the following transformation for the gauge fields

$$
A^{i}=g^{-1} A^{i} g+i g^{-1} \partial^{i} g
$$

As a result the new transition functions are given by

$$
\begin{aligned}
& \Omega_{1}^{\prime}\left(\sigma_{1}, \sigma_{2}\right)=g^{-1}\left(\sigma_{1}+2 \pi, \sigma_{2}\right) \Omega_{1}\left(\sigma_{1}, \sigma_{2}\right) g\left(\sigma_{1}, \sigma_{2}\right) \\
& \Omega_{2}^{\prime}\left(\sigma_{1}, \sigma_{2}\right)=g^{-1}\left(\sigma_{1}, \sigma_{2}+2 \pi\right) \Omega_{2}\left(\sigma_{1}, \sigma_{2}\right) g\left(\sigma_{1}, \sigma_{2}\right)
\end{aligned}
$$

Again all this is just as in the classical case except that one has to take into account the noncommutativity of the $\sigma_{i}$ 's.

It will be useful to consider first the $\Theta=0$ commutative case. Then one knows 
both the original gauge fields (2.14) and the transformed ones

$$
A^{\prime 1}=0, \quad A^{2}=\frac{m}{n} \frac{\sigma_{1}}{2 \pi}+Q
$$

where $Q=\frac{1}{n} \operatorname{diag}(0,1, \ldots, n-1)$, and I use primes for all variables in the new gauge. Than the differential equation for the gauge transformation is

$$
\partial^{1} g=0, \quad i \partial^{2} g=g A^{2}-A^{2} g=g Q
$$

which can be integrated to give

$$
g=K e^{-i \sigma_{2} Q}
$$

where the integration constant $K$ is the $n \times n$ matrix (2.20). It was fixed by requiring a trivial $\Omega_{2}^{\prime}$ as given by (3.19). Using the gauge transformation (3.20) one can can now calculate both transition functions

$$
\Omega_{1}^{\prime}=e^{2 \pi i m / n} e^{i \sigma_{2} T_{m}} V^{m}, \quad \Omega_{2}^{\prime}=1
$$

where $T_{k}=\operatorname{diag}(0, \ldots, 0,1, \ldots, 1), k=1, \ldots, n$ with the first $n-k$ entries vanishing and the last $k$ equal to unity.

Next I will discuss the noncommutative case. The first thing to notice is that the original quantum transition functions (2.18) are $\theta$ independent and only contain the $\sigma_{2}$ variable. Similarly the classical gauge transformation (3.20) only depends on $\sigma_{2}$ so that the classical computation of the new transition functions is also valid in the quantum case. Using (3.18) the new gauge fields are given by

$$
A^{\prime 1}=0, \quad A^{\prime 2}=\frac{m}{n+m \theta} \frac{\sigma_{1}}{2 \pi}+\frac{n}{n+m \theta} Q
$$


Since (3.16) implies $\left[\mathcal{U}_{i}, g\right]=0$ one can see that the gauge transformation is compatible with the quotient conditions (2.2). One can use the gauge transformation to obtain the generators of the sections of the adjoint bundle

$$
Z_{1}^{\prime}=e^{i \sigma_{1} /(n+m \theta)} e^{-2 \pi i n \theta^{\prime} Q}, \quad Z_{2}^{\prime}=e^{2 \pi i / n} V e^{i \sigma_{2}\left(1-T_{n-1}\right)}
$$

The explicit formulae for the fundamental sections in the new gauge is

$$
\Phi_{k}^{\prime}\left(\sigma_{1}, \sigma_{2}\right)=\sum_{r \in \mathbf{Z}} e^{i \sigma_{2} r} \chi_{k-n r}\left(\frac{\sigma_{1}}{2 \pi}+\frac{k-n r}{m}\right) .
$$

The $\chi_{s}$ functions are defined over the real axis and must satisfy

$$
\chi_{s+m}(x)=e^{-2 \pi i m / n} \chi_{s}(x),
$$

so that only $m$ of them are independent. Again, note that since the transition functions only contain $\sigma_{2}$ and all were ordered to the left of $\sigma_{1}$ in the solution for the sections of the fundamental bundle, they have the same form in the noncommutative and in the classical case. 


\section{Chapter 4}

\section{Adding Ramond-Ramond Backgrounds}

For $d \geq 2$ besides the compactification metric there are additional moduli which, in terms of the auxiliary Type IIA string theory [73, 69], correspond to the 2-form of the NS-NS (Neveu Schwarz-Neveu Schwarz) sector and the R-R (Ramond-Ramond) forms. Next I will extend the result of Chapter 2 by allowing arbitrary R-R backgrounds.

In Section $4.1 \mathrm{I}$ will review the transformation properties of the $\mathrm{R}-\mathrm{R}$ moduli under the duality group $[11,36,29]$. The dimensionally reduced action of Type IIA supergravity is invariant under the T-duality group ${ }^{1} S O(d, d)$. By deriving the nonlinear sigma model which describes the scalar fields of the supergravity, one can extract the transformation properties of the R-R backgrounds under the duality group. In particular I will show that appropriately defined fields, which are combinations of the R-R forms and the NS-NS two-form, transform in a spinor representation of the duality group.

In Section 4.2, I will identify the Chern-Simon parameters of the gauge theory with the $\mathrm{R}-\mathrm{R}$ moduli and then show that the duality transformations relating different NCSYM theories can be extended to include these terms. In the pro-

\footnotetext{
${ }^{1}$ The equations of motions are invariant under the full U-duality group $E_{d+1(d+1)}$.
} 
cess I will also obtain the transformation properties of the parameters and show that they coincide with the transformations expected from string theory and derived in Section 4.1 using the dimensional reduction of 10-dimensional Type IIA supergravity.

Finally in Appendix B I will present some results, used in the main text, regarding transformation properties under the T-duality group in the limit of small compactification volume and decoupling of string excitations.

A similar proposal for the additional terms in the noncommutative action were made in [41].

\subsection{Duality of Seven Dimensional Supergravity}

Type IIA superstring theory compactified on a d-dimensional torus is invariant under the T-duality group $S O(d, d \mid \mathrm{Z})$. The low energy supergravity effective action describing this compactification is in fact invariant under the continuous group $S O(d, d)$. This action can be obtained directly from the 10-dimensional Type IIA supergravity by dimensional reduction. In this section I will derive the transformation properties of the R-R moduli under the discrete duality group. Since this is a subgroup of the corresponding continuous group which is a symmetry of the low energy 10-dimensional supergravity action, one can obtain these transformation properties by analyzing the symmetries of the the nonlinear sigma model which describes the dynamics of the scalars in the supergravity action.

The NS-NS scalars are described locally by an $O(d, d) / O(d) \times O(d)$ nonlinear 
sigma model. Taking into account the T-duality group, the NS-NS nonlinear sigma model is in fact defined on

$$
O(d, d \mid \mathbf{Z}) \backslash O(d, d) / O(d) \times O(d)
$$

On the other hand simple counting arguments suggest that the R-R scalar fields transform in a chiral spinor representation of the duality group. This statement is almost correct except that the fields which transform in the spinor representation are some redefined fields involving not only the R-R fields but also the NS-NS two form.

The 10-dimensional supergravity action written in terms of the string metric is given by

$$
\begin{gathered}
\mathcal{S}=\int d^{10} x \sqrt{g} e^{-2 \phi}\left(R+4(\nabla \phi)^{2}-\frac{1}{2 \cdot 3 !} H^{2}\right) \\
-\int d^{10} x \sqrt{g}\left(\frac{1}{2 \cdot 2 !} F^{2}+\frac{1}{2 \cdot 4 !} F^{2}\right) \\
-\frac{1}{4} \int F_{(4)} \wedge F_{(4)} \wedge B+\ldots,
\end{gathered}
$$

where I have not written the terms containing the fermionic fields. The first line contains only NS-NS fields while the second contains the kinetic terms of the R-R forms. The various field strengths are defined as follows

$$
\begin{aligned}
H & =d B \\
F & =d A_{(1)}, \\
F_{(4)} & =d A_{(3)}, \\
F^{\prime} & =F_{(4)}+A_{(1)} \wedge d B,
\end{aligned}
$$


where the subscript indicates the rank of the form. Note that $R-R$ fields couple to the NS-NS fields through the metric and through the $F^{2}$ term, which depends on the antisymmetric NS-NS two-form.

Next I will perform the dimensional reduction along coordinates $x^{i}$ for $i=$ $1,2,3$. The massless scalars from the NS sector can be organized in the symmetric matrix [72]

$$
\mathcal{M}=\left(\begin{array}{cc}
G^{-1} & -G^{-1} B \\
B G^{-1} & G-B G^{-1} B
\end{array}\right) .
$$

Note that $\mathcal{M}$ is also an element of the group $S O(3,3)$. Using a result from Appendix $\mathrm{B}$, one can obtain the Weyl spinor representation of $\mathcal{M}$

$$
S(\mathcal{M})=\left(\begin{array}{cc}
\operatorname{det} G^{-1 / 2} & \operatorname{det} G^{-1 / 2} b^{T} \\
\operatorname{det} G^{-1 / 2} b & \operatorname{det} G^{1 / 2} G^{-1}+\operatorname{det} G^{-1 / 2} b b^{T}
\end{array}\right),
$$

where $b=* B$, and the star denotes the operator which transforms an antisymmetric matrix into its dual column matrix. The star operator always dualizes only with respect to the compactified coordinates.

One obtains additional scalars from the dimensional reduction of $\mathrm{R}-\mathrm{R}$ forms. As mentioned above these fields do not have simple transformation properties under the T-duality group but one can define the following odd rank forms

$$
\begin{aligned}
& C_{(1)}=A_{(1)}, \\
& C_{(3)}=A_{(3)}-A_{(1)} \wedge B,
\end{aligned}
$$

and organize them in a column matrix which, as will be seen shortly, transforms 
in a chiral spinor representation of the duality group

$$
\chi=\left(\begin{array}{c}
C_{123} \\
C_{1} \\
C_{2} \\
C_{3}
\end{array}\right) .
$$

The other fields can also be organized in representations of the duality group such that the action obtained by dimensional reduction from 10-dimensional supergravity is explicitly invariant. The six vectors obtained from the dimensional reduction of NS-NS fields transform in the fundamental representation while the 7-dimensional dilaton and the 7-dimensional space-time metric and 2-form are singlets. The four vectors obtained from the R-R forms transform in a chiral spinor representation and, after dualizing the 3 -form, the rest of the bosonic fields form a chiral spinor of 2 -forms.

For our purpose, it will be enough to consider the nonlinear sigma model part of the action containing the kinetic terms of the scalar fields of the theory

$$
\mathcal{S}=\frac{1}{2} \int d^{7} x \sqrt{\tilde{g}}\left(e^{-2 \Phi} \tilde{g}^{\mu \nu} \operatorname{tr}\left(\partial_{\mu} \mathcal{M}^{-1} \partial_{\nu} \mathcal{M}\right)+\tilde{g}^{\mu \nu} \partial_{\mu} \chi^{T} S(\mathcal{M}) \partial_{\nu} \chi\right)+\ldots
$$

where $\tilde{g}_{\mu \nu}$ and $\Phi$ are the 7-dimensional metric and dilaton, and I have not written the kinetic term for the dilaton. The nonlinear sigma model part of the action is written in a form that is explicitly invariant under $S O(3,3)$ and in fact the whole supergravity action could be written in invariant form. The duality transformations of the scalar fields are given by

$$
\begin{aligned}
\overline{\mathcal{M}} & =\Lambda^{-T} \mathcal{M} \Lambda^{-1} \\
\bar{\chi} & =S(\Lambda) \chi .
\end{aligned}
$$


To prove the invariance of the action I used $S\left(\Lambda^{T}\right)=S(\Lambda)^{T}$.

The main purpose of this section was to obtain the relations (4.2) which show how the fields $\chi$ with simple transformations properties under the T-duality group are related to the $\mathrm{R}-\mathrm{R}$ forms.

\subsection{T-duality of the Chern-Simon Type Terms}

In this section I will discuss how to modify the NCSYM action so that it describes the DLCQ of M-theory in the presence of arbitrary moduli. In the auxiliary Type IIA string theory the additional moduli are constant R-R backgrounds corresponding to generalized Wilson lines. Then I will show that the action which includes the new terms is also invariant under the duality group $S O(3,3 \mid \mathbf{Z})$ and that the parameters of the new terms transform exactly as expected from string theory.

First I will guess the form of these terms using our experience with the commutative case which corresponds to a vanishing NS-NS background 2-form $B$. In this case the compactified Matrix model corresponding to $n$ D0-branes is described by a $U(n)$ supersymmetric Yang-Mills theory. This is obtained by performing a T-duality transformation along all the compact directions. However, for nonvanishing R-R moduli, the action contains an additional Chern-Simon type term $[50,10,43,40,54]$

$$
\mathcal{S}_{C S}=\frac{1}{4(2 \pi)^{3}} \int \operatorname{tr}\left(e^{2 \pi \mathcal{F}} \sum_{k \text { odd }} A^{(k)}\right)
$$


where $A^{(k)}$ are the the T-dual R-R fields. Note also that under T-duality in all directions the dual of $B$ also vanishes if $B$ was zero. This is why only $\mathcal{F}$ appears in the exponent while in general one would also subtract the dual of $B$.

Next, consider the effect of a nonvanishing $B$ on this action. If $\gamma^{i j}$ represents a two cycle wrapped around directions $x^{i}$ and $x^{j}$, then the deformation parameters are defined by

$$
\Theta_{i j}=\frac{1}{(2 \pi)^{2}} \int_{\gamma^{i j}} B .
$$

In the super Yang-Mills part of the action the only change required by a nonvanishing $B$ was to make the coordinates noncommutative with deformation parameter $\Theta$. The metric and gauge coupling constant are the same as those obtained by T-duality from the Matrix model for a vanishing NS-NS 2-form. I emphasize that the metric of the NCSYM gauge theory is not the T-dual metric obtained by first taking the inverse of $E=G+\Theta$ and then extracting the symmetric part. The NCSYM metric $G^{i j}$ is just the inverse of the original metric. Thus one must distinguish between a T-duality in all directions and the noncommutative Fourier transformation relating the Matrix model and the NCSYM gauge theory.

Let us explain why the NCSYM metric is $\Theta$ independent. To compactify the Matrix model on a torus I will first consider the Matrix model on the covering space and then impose a quotient condition. If the $B$ modulus is nonvanishing, after going to the topologically trivial covering space, it can be gauged away. However this gauge transformation does not leave the wave functions of strings invariant and thus one must transform the translation operators implementing 
the quotient condition. The new translation operators do not commute and their noncommutativity is measured by $\Theta$.

Imposing the new quotient conditions on the Matrix action results directly in the NCSYM gauge theory. The only difference with the $B=0$ case is that one has to use noncommutative Fourier transformations instead of the standard Fourier transformations when going from the Matrix model to the NCSYM gauge theory. This however does not result in a different metric and gauge coupling constant. The main point of this discussion was to show that one can trade a nonvanishing $B$ field for noncommutative coordinates on the dual super Yang-Mills gauge theory.

I will assume that the parameters of the Chern-Simon terms are also the same as for vanishing $\Theta$, except that the new terms are defined on a noncommutative torus. In particular for compactification on a three torus one has

$$
\mathcal{S}_{C S}=\frac{1}{4(2 \pi)^{3}} \int \operatorname{tr}\left(2 \pi \mathcal{F} \wedge A^{(2)}+\frac{1}{2} 2 \pi \mathcal{F} \wedge 2 \pi \mathcal{F} \wedge A^{(0)}\right)
$$

Just as in the commutative case these terms are topological, supersymmetric and gauge invariant. In this action $\Theta$ only appears through the noncommutativity of the coordinates and $A^{(0)}$ and $A^{(2)}$ are the T-dual R-R forms ${ }^{2}$ calculated as if the NS-NS 2-form vanishes

$$
A^{(0)}=* A_{(3)}, \quad A^{(2)}=-* A_{(1)}
$$

The 1-form R-R field $A_{(1)}$ has a lower index and should not be confused with

\footnotetext{
${ }^{2}$ When I write the R-R forms in components I will drop the rank of the form as it is possible to identify the form from the position and number of indices.
} 
the Yang-Mills gauge field $A^{i}$. With this distinction in mind I will write the action (4.4) in the dual Matrix theory language using the R-R backgrounds on the original torus

$$
\mathcal{S}_{C S}=\int d t \operatorname{Tr}\left(\dot{X}^{i} A_{i}+\frac{i}{2 \pi} \dot{X}^{i} X^{j} X^{k} A_{i j k}\right),
$$

where $\operatorname{Tr}$ is the formal trace over infinite dimensional matrices divided by the infinite order of the quotient group [78]. It is convenient to write the action in component notation

$$
\mathcal{S}_{C S}=\frac{1}{2} \int d t \int \frac{d^{3} \sigma}{(2 \pi)^{3}} \operatorname{tr}\left(\varepsilon_{i j k}\left(2 \pi \mathcal{F}^{0 i} A^{j k}+(2 \pi)^{2} \mathcal{F}^{0 i} \mathcal{F}^{j k} A\right)\right)
$$

where the magnetic and electric field strengths.in the temporal gauge are

$$
\mathcal{F}^{0 i}=i\left[\partial^{0}, D^{i}\right], \quad \mathcal{F}^{i j}=i\left[D^{i}, D^{j}\right] .
$$

In the original conjecture [9], the large $N$ limit of Matrix theory describes the infinite momentum frame of M-theory. Large $N$ corresponds to a decompactification of the light-cone direction and in this limit the $\Theta$ and $A_{(1)}$ can be set to zero. Note that in M-theory these moduli correspond to $C_{-i j}$ and $g_{-i}$ and can be eliminated by a gauge transformation and a reparametrization when $x^{-}$is noncompact. In that case only the last term in (4.5) survives, the action becomes commutative and reduces to the action considered in $[54,55]$.

The action (4.5) is invariant under the $S O(3,3 \mid \mathrm{Z})$ duality group of the auxiliary string theory. Consider a Chern-Simon type action defined on a $\eta$-bundle. Here $\eta$ is a $S O(3,3 \mid \mathbf{Z})$ spinor containing the rank of the group and the magnetic flux numbers. I will perform the same sequence of field redefinitions used in [14], 
where it was shown explicitly for the case of vanishing R-R moduli, that the $U(n)$ NCSYM action is equivalent to a $U(q)$ NCSYM action on a trivial bundle, where $q$ is the greatest common divisor of $n$ and the magnetic fluxes $M$. Let $H=(\mathcal{C} \Theta+\mathcal{D})^{-1}$ be the matrix defined in [14], where $\mathcal{C}$ and $\mathcal{D}$ are the lower block components of the $S O(3,3 \mid \mathrm{Z})$ transformation relating the original NCSYM gauge theory to the theory on the trivial bundle with $U(q)$ gauge group. Then one can make the following constant curvature connection and field redefinitions

$$
\begin{gathered}
\widehat{\nabla}^{i} \stackrel{\text { def }}{=}\left(H^{-1}\right)_{j}^{i} \nabla^{j}, \widehat{A}^{i} \stackrel{\text { def }}{=}\left(H^{-1}\right)_{j}^{i} A^{j}, \\
\widehat{D}^{i} \stackrel{\text { def }}{=}\left(H^{-1}\right)_{j}^{i} D^{j}, \\
\widehat{\mathcal{F}}^{k l}=\left[\widehat{\nabla}^{k}, \widehat{A}^{l}\right]-\left[\widehat{\nabla}^{l}, \widehat{A}^{k}\right]-i\left[\widehat{A}^{k}, \widehat{A}^{l}\right] .
\end{gathered}
$$

The curvature can be split into a constant term and a fluctuating piece

$$
\begin{gathered}
\mathcal{F}^{i j}=\mathcal{F}_{(0)}^{i j}+H_{k}^{i} H_{l}^{j} \widehat{\mathcal{F}}^{k l}, \\
\mathcal{F}^{0 k}=H_{l}^{k} \widehat{\mathcal{F}}^{0 l} .
\end{gathered}
$$

Using the matrices $Q$ and $R$ defined in [14] one can perform a change of integration variables $\widehat{\sigma}=\sigma Q R$, which introduces a Jacobian factor

$$
\int d^{3} \sigma \operatorname{tr} \Psi(\sigma)=\int d^{3} \widehat{\sigma} \operatorname{det}\left(Q^{-1}\right) \operatorname{tr} \Psi\left(\widehat{\sigma}(Q R)^{-1}\right)
$$

Making the substitutions (4.6), (4.7) and collecting similar terms one finds

$$
\mathcal{S}_{C S}=\frac{1}{2(2 \pi)^{2}} \int d t \int d^{3} \widehat{\sigma} \frac{q}{n} \operatorname{tr}\left(\varepsilon_{i j k}\left(\widehat{\mathcal{F}}^{0 i} A^{\prime j k}+2 \pi \widehat{\mathcal{F}}^{0 i} \widehat{\mathcal{F}}^{j k} A^{\prime}\right)\right)
$$


where

$$
\begin{gathered}
q A^{\prime}=\left(n \operatorname{det} Q^{-1} \operatorname{det} H\right) A \\
\varepsilon_{i j k} q A^{\prime i j}=H_{k}^{l} \varepsilon_{i j l} n \operatorname{det} Q^{-1}\left(A^{i j}+2 \pi \mathcal{F}_{(0)}^{i j} A\right)
\end{gathered}
$$

One can now rewrite the action in terms of new operators $\sigma_{i}^{\prime}, \partial^{\prime i}$, and $U^{\prime}$, and a $q$ dimensional trace. See [14] for a more detailed discussion of this substitution.

$$
\mathcal{S}_{C S}=\frac{1}{4(2 \pi)^{3}} \int \operatorname{tr}_{q}\left(2 \pi \mathcal{F}^{\prime} \wedge A^{\prime(2)}+\frac{1}{2} 2 \pi \mathcal{F}^{\prime} \wedge 2 \pi \mathcal{F}^{\prime} \wedge A^{\prime(0)}\right)
$$

More generally the action is invariant under duality transformations if the Chern-Simon parameters are related as follows

$$
\begin{gathered}
\bar{A}_{(0)}=|\operatorname{det}(\mathcal{C} \Theta+\mathcal{D})|^{-1 / 2} A_{(0)} \\
*\left(\bar{A}^{(2)}+2 \pi \overline{\mathcal{F}}_{(0)} \bar{A}^{(0)}\right) \bar{n} \operatorname{det} \bar{Q}^{-1}=(\mathcal{C} \Theta+\mathcal{D})^{-T} *\left(A^{(2)}+2 \pi \mathcal{F}_{(0)} A^{(0)}\right) n \operatorname{det} Q^{-1}
\end{gathered}
$$

where $\mathcal{C}$ and $\mathcal{D}$ are the lower block components of the $S O(3,3 \mid \mathrm{Z})$ matrix relating the two theories, and the star operator is the duality operator acting only with respect to the compact coordinates.

Next I will write the Chern-Simon parameters in term of the fields $C$ discussed in Section 4.1

$$
\begin{gathered}
A^{(0)}=*\left(C_{(3)}+C_{(1)} \wedge \Theta\right) \\
A^{(2)}=-* C_{(1)} .
\end{gathered}
$$

To obtain a compact form first define the column matrices $u$ and $v$ with components

$$
u_{i}=\frac{1}{2} M^{j k} C_{i j k}-n C_{i}
$$




$$
v^{i}=-M^{i j} C_{j} .
$$

If $\chi$ transforms as a spinor, $u$ and $v$ are the block components of a $S O(3,3 \mid \mathrm{Z})$ vector as shown in the Appendix (B.8). Then using the identities (2.50) listed in Chapter 2, the transformation (4.8) can be written as

$$
\begin{gathered}
\bar{C}_{(3)}+\bar{C}_{(1)} \wedge \bar{\Theta}=|\operatorname{det}(\mathcal{C} \Theta+\mathcal{D})|^{-1 / 2}\left(C_{(3)}+C_{(1)} \wedge \Theta\right) \\
(\bar{u}-\bar{\Theta} \bar{v})=(\mathcal{C} \Theta+\mathcal{D})^{-T}(u-\Theta v) .
\end{gathered}
$$

Comparing (4.11) and (4.12) with (B.6) and (B.4) in Appendix B one sees that the R-R fields must transform in a spinor representation of $S O(3,3 \mid \mathrm{Z})$

$$
\bar{\chi}=S(\Lambda) \chi .
$$

Thus the duality transformations of all the parameters of the NCSYM, including those of the Chern-Simon type terms, coincide with the transformation of moduli of the Type IIA strings compactified on a torus in the limit of vanishing $\alpha^{\prime}$ and $G_{i j}$.

Using the transformation properties of $g_{S Y M}^{-2}$ and $A^{(0)}$ it follows that the complex coupling

$$
\tau=A^{(0)}+\frac{4 \pi i}{g_{S Y M}^{2}}
$$

also transforms simply under the T-duality group with the same $\Theta$ dependent factor appearing in (4.8).

Finally note that the BPS spectrum corresponding to the electric fluxes ob- 
tained in [15] is modified in the presence of nonvanishing R-R moduli

$$
\begin{gathered}
\mathcal{E}^{U(n)}=\frac{g_{S}}{2}\left|n-\frac{1}{2} \operatorname{tr}(M \Theta)\right|^{-1} \\
\times\left(\left(n_{i}-u_{i}\right)-\Theta_{i k}\left(m^{k}-v^{k}\right)\right) G^{i j}\left(\left(n_{j}-u_{j}\right)-\Theta_{j l}\left(m^{l}-v^{l}\right)\right) .
\end{gathered}
$$

This result agrees with the small volume limit of the spectrum formula in [41] and reduces for vanishing $\Theta_{i j}$ to the result of $[56,55]$. In [48] it was shown that shifts in the electric flux spectrum correspond to inequivalent geometric quantizations. These different quantizations are equivalent to the standard canonical quantization if one also includes topological terms in the action. 


\section{Chapter 5}

\section{T-duality of the BPS Spectrum}

As shown in Chapter 2 the action of a $U(n)$ NCSYM, with magnetic fluxes $M^{i j}$, can be written as the action of a $U(q)$ NCSYM on a trivial quantum bundle, where $q$ is the greatest common divisor of $n$ and $M^{i j}$. Thus these two theories must have identical spectra. The action contains magnetic backgrounds which I chose as in [14] so that the vacuum energy vanishes.

$$
\begin{aligned}
\mathcal{S}^{U(n)}= & \frac{1}{g_{S Y M}^{2}} \int d t \int d^{d} \sigma \sqrt{\operatorname{det}\left(G^{k l}\right)} \operatorname{tr}\left(\frac{1}{2} G_{i j} \mathcal{F}^{0 i} \mathcal{F}^{0 j}-\right. \\
& \frac{1}{4} G_{i j} G_{k l}\left(\mathcal{F}^{i k}-\mathcal{F}_{(0)}^{i k}\right)\left(\mathcal{F}^{j l}-\mathcal{F}_{(0)}^{j l}\right)+ \\
& \frac{1}{2} \sum_{a} \dot{X}^{a} \dot{X}^{a}-\frac{1}{2} \sum_{a} G_{i j}\left[D^{i}, X^{a}\right]\left[D^{j}, X^{a}\right]+ \\
& \left.\frac{1}{4} \sum_{a, b}\left[X^{a}, X^{b}\right]\left[X^{a}, X^{b}\right]+\text { fermions }\right) .
\end{aligned}
$$

All the equations in this chapter where $d$ is unspecified, are valid for the two and three dimensional case, but some may have to be modified in higher dimensions. For simplicity I will consider the case when $n$ and $M^{i j}$ are relatively prime. Then one can find a duality transformation $\Lambda$ such that $\bar{n}=1$ and $\bar{M}=0$ as was shown in [14]. From this point on, when I discuss the $U(n)$ theory I will use the the $d$-dimensional block matrices (2.42), with $\Lambda$ the particular transformation that takes the $U(n)$ theory into a $U(1)$ theory. For example the constant background 
field strength can be expressed in terms of the block components of $\Lambda$ as

$$
\mathcal{F}_{(0)}=\frac{-1}{2 \pi}(\mathcal{C} \Theta+\mathcal{D})^{-1} \mathcal{C} .
$$

One can write the connection as a sum of a constant curvature $U(1)$ connection $\nabla_{i}$, a zero mode $A_{(0)}^{i}$, and a fluctuating part $A^{i}$

$$
\nabla^{i}-i A_{(0)}^{i} \mathbf{1}-i A^{i}\left(Z_{j}\right)=\partial^{i}+i F^{i j} \sigma_{j}-i A_{(0)}^{i} \mathbf{1}-i A^{i}\left(Z_{j}\right) .
$$

Note that $A^{i}$ does not contain the zero mode. The $Z_{i}$ 's are $n$-dimensional matrices which generate the algebra of adjoint sections. For example, in the two dimensional case one has $[22,24,39,52,14]$

$$
Z_{1}=e^{i \sigma_{i} Q_{1}^{i} / n} V^{b}, Z_{2}=e^{i \sigma_{i} Q_{2}^{i} / n} U
$$

where $U$ and $V$ are the clock and shift matrices and $Q$ is a two dimensional matrix which reduces to the identity in the commutative case. Substituting this in the action gives

$$
\mathcal{S}^{U(n)}=\frac{1}{g_{S Y M}^{2}} \int d t \int d^{d} \sigma \sqrt{\operatorname{det}\left(G^{k l}\right)} \operatorname{tr} \frac{1}{2} G_{i j} \partial^{0} A_{(0)}^{i} \partial^{0} A_{(0)}^{j}+\ldots
$$

where the dots stand for terms containing only $A^{i}$. Thus classically the zero modes decouple, and the action is just that of a free particle

$$
\mathcal{S}_{(0)}^{U(n)}=\int d t \frac{(2 \pi)^{2}}{2} \mathcal{M}_{i j} \dot{A}_{(0)}^{i} \dot{A}_{(0)}^{j}
$$

where the mass matrix is given by

$$
\mathcal{M}_{i j}=\left|n-\frac{1}{2} \operatorname{tr}(M \Theta)\right| \frac{(2 \pi)^{d-2} \sqrt{\operatorname{det}\left(G^{k l}\right)}}{g_{S Y M}^{2}} G_{i j} .
$$


In the commutative case the first factor on the right hand side of (5.3) reduces to $n$ and arises from taking the trace. The origin of this factor in the noncommutative case was discussed in $[24,14]$. The corresponding Hamiltonian is then ${ }^{1}$

$$
\mathcal{H}_{(0)}^{U(n)}=\frac{1}{2} \mathcal{M}^{i j} E_{i}^{(0)} E_{j}^{(0)},
$$

where $\mathcal{M}^{i j}$ is the inverse mass matrix and $E_{i}^{(0)}$ is the momentum conjugate to $A_{(0)}^{i}$

$$
E_{i}^{(0)}=\frac{1}{2 \pi i} \frac{\partial}{\partial A_{(0)}}
$$

Note that $E_{i}^{(0)}$ correspond to zero modes of the electric field.

\subsection{The $U(1)$ BPS Spectrum}

Before calculating the spectrum of (5.4) directly, I will use the duality invariance of the spectrum and obtain it by using the simpler dual $U(1)$ theory. I will use primes for all the variables in the $U(1)$ theory. In this case the mass matrix takes the form

$$
\mathcal{M}_{i j}^{\prime}=\frac{(2 \pi)^{d-2} \sqrt{\operatorname{det}\left(G^{\prime k l}\right)}}{g^{\prime 2}{ }_{S Y M}^{\prime}} G_{i j}^{\prime}
$$

Just as in the commutative $U(1)$ supersymmetric gauge theory [85] the zero modes live on a torus. To see this consider the gauge transformations

$$
U_{i}^{\prime}=e^{i \sigma_{i}^{\prime}}
$$

These gauge transformations are single valued and leave the trivial transition functions invariant. Under these gauge transformations the connection transforms

\footnotetext{
${ }^{1}$ This only includes the energy coming form the electric zero modes.
} 


$$
U_{j}^{\prime-1}\left(\partial^{i i}-i A_{(0)}^{\prime i}-i A^{\prime i}\left(U_{k}^{\prime}\right)\right) U_{j}^{\prime}=\partial^{\prime i}-i\left(A_{(0)}^{\prime i}-\delta_{j}^{i}\right)-i A^{\prime i}\left(e^{-2 \pi i \Theta_{j k}^{\prime}} U_{k}^{\prime}\right)
$$

For vanishing $\Theta^{\prime}$ the effect of these gauge transformations is just a shift of the zero mode and one has the following gauge equivalences $A_{(0)}^{i} \sim A_{(0)}^{i}+\delta_{j}^{i}$. Note that $\delta_{(j)}^{i}$ for $j=1, \ldots, d$ form a basis for a lattice $L^{\prime}$ and the configuration space is $\mathbf{R}^{d} / L^{\prime}$. The conjugate momenta are then quantized

$$
E_{i}^{\prime(0)}=n_{i}^{\prime}
$$

and the spectrum of zero modes is then given by

$$
\mathcal{E}^{U(1)}=\frac{1}{2} \mathcal{M}^{i j} n_{i}^{\prime} n_{j}^{\prime}
$$

However in the noncommutative case one can see that the above gauge transformations also produces a translation in the $k$ direction proportional to $\Theta_{j k}^{\prime}$. This results in a modification of the spectrum similar to the Witten-Olive effect [84]. Let as define the total momentum operator operators $P_{i}^{\prime}$ such that

$$
\left[P^{\prime i}, \Psi\right]=-i \partial^{\prime i} \Psi
$$

where $\Psi$ is an arbitrary field of the theory. The momentum $P^{\prime i}$ defined by (5.6) is not the standard gauge invariant total momentum but the difference between the two is the generator of a gauge transformation with the gauge parameter equal to the $i$-component of the gauge field. Thus on gauge invariant states the total momentum defined above and the gauge invariant momentum have the same effect. 
The operator generating the gauge transformation is $[41,42]$

$$
\exp \left(2 \pi i\left(E_{j}^{\prime(0)}+\Theta_{j k}^{\prime} P^{\prime k}\right)\right)
$$

Translation by an integral number of periods on a trivial bundle must leave the physical system invariant. The operators generating these translations are given by

$$
\exp \left(2 \pi i P^{\prime k}\right)
$$

The operators (5.7) and (5.8) act as the identity on physical states so one obtains the quantization

$$
E_{j}^{\prime(0)}+\Theta_{j k}^{\prime} P^{\prime k}=n_{j}^{\prime}, \quad P^{\prime j}=m^{\prime j}
$$

where $n_{j}$ and $m^{j}$ are integers. The spectrum of zero modes is then given by

$$
\mathcal{E}^{U(1)}=\frac{1}{2} \mathcal{M}^{\prime i j}\left(n_{i}^{\prime}-\Theta_{i k}^{\prime} m^{\prime k}\right)\left(n_{j}^{\prime}-\Theta_{j l}^{\prime} m^{\prime \prime}\right) .
$$

This result has the following geometric interpretation. In the sectors of nonvanishing momentum the wave function for the zero modes is not strictly speaking a function but rather a section on a twisted bundle over the torus $\mathbf{R}^{d} / L^{\prime}$ with twists given by $\exp \left(\Theta_{i k}^{\prime} m^{\prime k}\right)$.

\subsection{Dual $U(n)$ BPS Spectrum}

Using the duality transformations (1.12) I can express the spectrum in terms of the $U(n)$ parameters

$$
\mathcal{E}^{U(n)}=\frac{1}{2} \frac{g_{S Y M}^{2}}{(2 \pi)^{d-2} \sqrt{\operatorname{det}\left(G^{i j}\right)}}|\operatorname{det}(\mathcal{C} \Theta+\mathcal{D})|^{-1 / 2}
$$




$$
\times G^{i j}\left(n_{i}-\Theta_{i k} m^{k}\right)\left(n_{j}-\Theta_{j k} m^{k}\right)
$$

where I also performed a duality transformation on the quantum numbers [41, 42]

$$
\left(\begin{array}{c}
n_{i}^{\prime} \\
m^{\prime i}
\end{array}\right)=\left(\begin{array}{cc}
\mathcal{A} & \mathcal{B} \\
\mathcal{C} & \mathcal{D}
\end{array}\right)\left(\begin{array}{c}
n_{i} \\
m^{i}
\end{array}\right) .
$$

Next consider in more detail the two dimensional case. The parameters of the $U(1)$ and $U(n)$ NCSYM are related by the $S O(2,2 \mid \mathbf{Z})$ transformation [14]

$$
\Lambda=\left(\begin{array}{cc}
a I_{2} & b \varepsilon \\
-m \varepsilon & n I_{2}
\end{array}\right),
$$

where $\varepsilon$ is a two dimensional matrix with the only nonvanishing entries given by $\varepsilon_{12}=-\varepsilon_{21}=1$. In this case $(\mathcal{C} \Theta+\mathcal{D})_{j}^{i}=(n+\theta m) \delta_{j}^{i}$ and the spectrum is

$$
\mathcal{E}^{U(n)}=\frac{1}{2} \frac{g_{S Y M}^{2}}{(2 \pi)^{d-2}|n+\theta m| \sqrt{\operatorname{det}\left(G^{k l}\right)}} G^{i j}\left(n_{i}+\theta m_{i}\right)\left(n_{j}+\theta m_{j}\right)
$$

where $m^{i}=\varepsilon^{i j} m_{j}$. This result ${ }^{2}$ has the expected factor of $|n+\theta m|$ in the denominator. In the DLCQ formulation of $\mathrm{M}$ theory this factor is proportional to the kinetic momentum in the compact light-like direction and is expected to appear in the denominator of the DLCQ Hamiltonian.

Next the spectrum is obtained directly in the $U(n)$ theory. I will do this in two ways. First, consider the generators of the adjoint algebra, the $Z_{i}$ 's. These generators satisfy

$$
Z_{k}\left(\sigma_{i}+2 \pi \delta_{i}^{j}\right)=\Omega_{j} Z_{k}\left(\sigma_{i}\right) \Omega_{j}^{-1} .
$$

\footnotetext{
${ }^{2}$ Expressed in terms of the string coupling constant of the auxiliary string theory the spectrum takes the simpler form $E^{U(n)}=\frac{1}{2} g_{s}|n+\theta m|^{-1} G^{k l}\left(n_{k}+\theta m_{k}\right)\left(n_{l}+\theta m_{l}\right)$.
} 
Besides having the privileged role of generators for the sections of the adjoint bundle, the $Z_{i}$ 's can also be used to perform gauge transformations since they are unitary. Next, rewrite (5.12) as

$$
\Omega_{j}=Z_{k}\left(\sigma_{i}+2 \pi \delta_{i}^{j}\right)^{-1} \Omega_{j} Z_{k}\left(\sigma_{i}\right)
$$

The right hand side of (5.13) gives the transformation of the transition functions under the $Z_{i}$ gauge transformation. One can see that, just as in the $U(1)$ case where the gauge transformations $U_{i}^{\prime}$ left the transition functions trivial, the $Z_{i}^{\prime}$ 's leave the transition functions invariant. Following the same strategy as in the $U(1)$ case, where I used the $U_{i}^{\prime}$ to find the configuration space of the zero modes, I will use here $Z_{i}$

$$
\begin{gathered}
Z_{j}^{-1}\left(\nabla^{i}-i A_{(0)}^{i} \mathbf{1}-i A^{i}\left(Z_{k}\right)\right) Z_{j}= \\
\nabla^{i}-i\left(A_{(0)}^{i}-\left((\mathcal{C} \Theta+\mathcal{D})^{-1}\right)_{j}^{i}\right) \mathbf{1}-i A^{i}\left(e^{-2 \pi i \Theta_{j k}^{\prime}} Z_{k}\right) .
\end{gathered}
$$

Note that again I have separated the zero mode of the gauge connection and I have used the identity [14]

$$
\left[\nabla^{i}, Z_{j}\right]=i\left((\mathcal{C} \Theta+\mathcal{D})^{-1}\right)_{j}^{i} Z_{j}
$$

One can express the gauge transformed connection as

$$
e^{-2 \pi(\mathcal{A} \Theta+\mathcal{B})_{j k} \nabla^{k}}\left(\nabla^{i}-i A_{(0)}^{i} \mathbf{1}-i A^{i}\left(Z_{k}\right)\right) e^{2 \pi(\mathcal{A} \Theta+\mathcal{B})_{j k} \nabla^{k}}+i \mathcal{A}_{j}^{i} \mathbf{1}
$$

where I used

$$
(\mathcal{C} \Theta+\mathcal{D})^{-1}=\left(\mathcal{A}-\Theta^{\prime} \mathcal{C}\right)^{T}
$$


and (5.2) to rewrite the extra shift in the zero mode.

Next I will define the momentum operator by its action on the fields of the theory. For example on the gauge fields $P^{i}$ acts as

$$
\left[P^{i}, A_{(0)}^{j} \mathbf{1}+A^{j}\left(Z_{k}\right)\right]=-i\left[\nabla^{i}, A^{j}\left(Z_{k}\right)\right]-i \mathcal{F}_{(0)}^{i j}
$$

Note that $P^{i}$ also acts on the zero mode $A_{(0)}^{i}$. This can be understood as follows. When defining the momentum there is a choice whether to include as part of the system the magnetic background $\mathcal{F}_{(0)}^{i j}$. The standard gauge invariant momentum for which the momentum density is $\operatorname{tr}\left(F^{i j} E_{j}\right)$ can be written as the sum of two terms. The first is just the momentum translating the part of the system that does not include the magnetic background and whose momentum density is $\operatorname{tr}\left(\left(F^{i j}-\right.\right.$ $\left.\left.F_{(0)}^{i j}\right) E_{j}\right)$. The second term is an operator shifting the zero mode of the gauge field as in (5.15). Then our $P^{i}$ can be identified, up to the generator of a gauge transformation, with the total momentum that includes the magnetic background. Furthermore, one can identify, up to the generator of a gauge transformation, the first term on the right hand side of (5.15) as the action of the momentum operator that translates only the fluctuating part. As will be seen later it is the momentum whose density is $\operatorname{tr}\left(F^{i j} E_{j}\right)$ that appears in the $S O(d, d \mid \mathbf{Z})$ duality transformation.

A convenient way of writing the action of $P^{i}$ on the gauge field is

$$
\left[P^{i},-i A_{(0)}^{j} \mathbf{1}-i A^{j}\left(Z_{k}\right)\right]=-i\left[\nabla^{i}, \nabla^{j}-i A_{(0)}^{j} \mathbf{1}-i A^{j}\left(Z_{k}\right)\right]
$$

Then using (5.14) one sees that the quantum operator which implements the gauge 
transformation above is given by

$$
\exp \left(2 \pi i\left(\mathcal{A}_{j}^{k}\left(E_{k}^{(0)}+\Theta_{k l} P^{l}\right)+\mathcal{B}_{j k} P^{k}\right)\right)
$$

The momentum operator $P^{i}$ has integer eigenvalues since the space is a torus with lengths $2 \pi$. One can also see this by considering the operator

$$
\exp \left(2 \pi i\left(\mathcal{C}^{j i} E_{i}^{(0)}+\left(\mathcal{C}^{j k} \Theta_{k i}+\mathcal{D}_{i}^{j}\right) P^{i}\right)\right)
$$

This acts trivially on every operator in the $U(n)$ theory. In particular the combination of operators in the exponent has no effect on the zero mode. The condition that (5.16) and (5.17) should act as the identity on the physical Hilbert space is equivalent to the quantization

$$
\begin{aligned}
& \mathcal{A}_{j}^{k}\left(E_{k}^{(0)}+\Theta_{k l} P^{l}\right)+\mathcal{B}_{j k} P^{k}=n_{j}^{\prime} \\
& \mathcal{C}^{j k}\left(E_{k}^{(0)}+\Theta_{k l} P^{l}\right)+\mathcal{D}_{k}^{j} P^{k}=m^{\prime j} .
\end{aligned}
$$

Since the matrices $\mathcal{A}, \mathcal{B}, \mathcal{C}$, and $\mathcal{D}$ are the block components of an element of $S O(d, d \mid \mathbf{Z})$ this is equivalent to

$$
E_{j}^{(0)}+\Theta_{j k} P^{k}=n_{j}, \quad P^{j}=m^{j}
$$

where $n_{j}$ and $m^{j}$ are integers. Using the Hamiltonian (5.4) and the above quantization the electric flux spectrum is

$$
\begin{aligned}
\mathcal{E}^{U(n)}= & \frac{1}{2} \frac{g_{S Y M}^{2}}{(2 \pi)^{d-2} \sqrt{\operatorname{det}\left(G^{i j}\right)}}\left|n-\frac{1}{2} \operatorname{tr}(M \Theta)\right|^{-1} \\
& \times G^{i j}\left(n_{i}-\Theta_{i k} m^{k}\right)\left(n_{j}-\Theta_{j k} m^{k}\right)
\end{aligned}
$$


which is identical to the result (5.9) obtained by duality.

Finally I will present an alternative derivation of the spectrum using the gauge transformations $\exp \left(i \bar{\sigma}_{i}\right)$ where $\bar{\sigma}_{i}=\sigma_{j} Q_{i}^{j}$ and $Q_{i}^{j}$ is a matrix defined in [14] and equals the identity for vanishing deformation parameter or magnetic background. This derivation is closely related to the derivation of the spectrum in [41, 42]. As discussed in [14] gauge invariant quantities such as the Lagrangian density have periodicity $2 \pi$ in the $\bar{\sigma}_{i}$ variables. Then one can use $\bar{U}_{i}=\exp \left(i \bar{\sigma}_{i}\right)$ as a gauge transformation just as one used $U_{i}^{\prime}$ in the $U(1)$ theory. Note first that $\bar{U}_{i}$ is a globally defined gauge transformation. It is convenient to write it as $\bar{U}_{i}=\mathcal{U}_{i} e^{2 \pi \Theta_{i j} \nabla^{j}}$. Here $\mathcal{U}_{i}=e^{i \sigma_{i}-2 \pi \Theta_{i j} \partial^{j}}$ and is the variable implementing the quotient condition [24]. The effect of this gauge transformation is

$$
\begin{gathered}
\bar{U}_{j}^{-1}\left(\nabla^{i}-i A_{(0)}^{i} \mathbf{1}-i A^{i}\left(Z_{k}\right)\right) \bar{U}_{j}= \\
e^{-2 \pi \Theta_{i j} \nabla^{j}}\left(\nabla^{i}-i A_{(0)}^{i} \mathbf{1}-i A^{i}\left(Z_{k}\right)\right) e^{2 \pi \Theta_{i j} \nabla^{j}}+i \delta_{j}^{i} .
\end{gathered}
$$

The operator implementing this gauge transformation in the Hilbert space is

$$
\exp \left(-2 \pi i\left(E_{k}^{(0)}+\Theta_{k l} P^{l}\right)\right)
$$

Again, on gauge invariant states this operator acts trivially and together with the quantization of the momentum results in the same spectrum (5.18) as using $Z_{i}$. Note that the second method of deriving the $U(n)$ spectrum is similar in spirit to the derivation of the $U(1)$ spectrum. For example the gauge transformation is an element of the $U(1)$ subgroup. However, the first derivation is instructive since it exhibits inside the $U(n)$ theory the dual $U(1)$ theory variables such as $P^{i}$ and $E_{i}^{\prime}$. 


\section{Chapter 6}

\section{Path Integral Quantization of the Symplectic Leaves of the $S U(2)^{*}$ Poisson-Lie Group}

As briefly discussed in the introduction, in this chapter I will use the Feynman path integral to quantize the symplectic leaves of $S U(2)^{*}$. In doing this I will follow closely the method used in [53]. In fact, a strong parallel exists both at the classical and the quantum levels. Classically, the leaves coincide in the trivial and Poisson cases once expressed in terms of Darboux coordinates. Consequently, at the quantum level we have the same Hilbert space and the two quantum algebras are isomorphic. The path integral has the same form in the trivial and Poisson cases, but one has to insert different functions to obtain $s u(2)$ or $\mathcal{U}_{q}(s u(2))$ generators.

In Section 6.1, I review some general Poisson-Lie theory mainly to fix the notation and to list some results used later in the chapter. The results in this section are given using complex coordinates. In Section 6.2 , I describe the reality structures of $S U(2)$, its dual and its double. I also give a detailed description of the symplectic leaves of $S U(2)^{*}$.

In Section 6.3, I describe Darboux coordinates, formulate the path integral and find the radius quantization condition using a quantization condition similar 
to [53]. I also define the Hilbert space and obtain the matrix elements of diagonal operators. In Section 6.4, I study general matrix elements and show that they are representations of the quantum group algebra. In the last section I draw some conclusions and suggest how this work might be generalized. Finally, I will review the isomorphism of $F u n_{q}\left(S U(2)^{*}\right)$ and $\mathcal{U}_{q}(s u(2))$ and the derivation of the Poisson bracket on $S U(2)^{*}$ from $F u n_{q}\left(S U(2)^{*}\right)$ in Appendix C.

\subsection{Dual Pairs of Poisson-Lie Groups}

A Poisson-Lie Group (PLG) is a pair $(G,\{\}$,$) where G$ is a Lie group and $\{$,$\} is a Poisson bracket on G$ which is compatible with the group operations of multiplication and inversion [28]. The compatibility determines the Poisson structure at an arbitrary point from its values in the vicinity of the group unit. A PLG can be equivalently described as a Poisson Hopf algebra Fun $(G)$ which is a commutative Hopf algebra with a compatible Poisson algebra. In what follows I will freely exchange these two dual descriptions.

The Poisson bracket on the group determines a Lie algebra structure on the cotangent space $g^{*}$ of the Lie group. Let $h_{1}$ and $h_{2}$ be two functions on the group G. Then:

$$
\left[d h_{1}, d h_{2}\right]^{*} \equiv d\left\{h_{1}, h_{2}\right\}
$$

defines a Lie algebra $\left(g^{*},[,]^{*}\right)$. One can check that this definition is independent of the choice of functions used to represent cotangent vectors. Let $\left\{e_{i}\right\}$ be a basis of 
$g,\left\{e^{i}\right\}$ its dual basis in $g^{*}$, and $f_{i j}^{k}$ and $\tilde{f}_{c}^{a b}$ the corresponding structure constants. The compatibility of the Poisson and group structures imposes restrictions on the two Lie algebras. In terms of the structure constants, they read

$$
f_{i j}^{s} \tilde{f}_{s}^{a b}-f_{i s}^{a} \tilde{f}_{j}^{s b}+f_{i s}^{b} \tilde{f}_{j}^{s a}-f_{j s}^{b} \tilde{f}_{i}^{s a}+f_{j s}^{a} \tilde{f}_{i}^{s b}=0 .
$$

In fact, similarly to a Lie group being determined up to some global features by its Lie algebra, a PLG is in one to one correspondence with a Lie bialgebra (LBA). This is a pair $\left(g, g^{*}\right)$ of Lie algebras dual as vector spaces whose structure constants satisfy (6.1). Note that the LBA structure is symmetric between $g$ and $g^{*}$, so to each LBA we can associate a pair of PLGs $G$ and $G^{*}$.

An equivalent definition of a LBA is given in terms of the cocommutator $\delta$ the dual of the []$^{*}$ commutator

$$
\delta: g \rightarrow \wedge^{2} g,\langle\delta(x), \xi \wedge \eta\rangle=\left\langle x,[\xi, \eta]^{*}\right\rangle, x \in g, \xi, \eta \in g^{*}
$$

Jacobi for [, ${ }^{*}$ implies co-Jacobi $(\delta \otimes i d) \circ \delta=0$. The compatibility condition (6.1) translates into the cocycle condition

$$
\delta([x, y])=[\Delta(x), \delta(y)]+[\delta(x), \Delta(y)]
$$

where $\Delta(x)=x \otimes 1+1 \otimes x$ and similarly for $y$.

A quasi-triangular Lie bialgebra is a LBA such that there exists a $r \in g \otimes g$ which, for all $x \in g$ satisfies:

1. $\delta(x)=[r, \Delta(x)]$; 
2. $I=r+\sigma(r)$ is adjoint invariant $[I, \Delta(x)]=0$. Here $\sigma$ is the permutation operator;

3. $(\delta \otimes i d) r=\left[r_{13}, r_{23}\right],(i d \otimes \delta) r=\left[r_{13}, r_{12}\right]$.

A factorizable Lie bialgebra is a quasi-triangular LBA such that $I$ is nondegenerate. One can use $I$ to identify $g$ and $g^{*}$. The factorization refers to the fact that any $x \in g$ can be decomposed as $x=x_{+}-x_{-}$. Here

$$
x_{+}=\langle r, \xi \otimes i d\rangle, x_{-}=-\langle r, i d \otimes \xi\rangle
$$

for some $\xi \in g^{*}$ satisfying $x=\langle I, \xi \otimes i d\rangle$. Such a $\xi$ always exists since $I$ is non-degenerate.

A PLG $G$ is quasi-triangular if its tangent LBA $g$ is quasi-triangular. Similarly a PLG is factorizable if its tangent LBA is factorizable.

One can define two important Poisson brackets $\{,\}_{ \pm}$on a quasi-triangular LBA.

$$
\{f, h\}_{ \pm}=\langle r, \nabla f \otimes \nabla h\rangle \pm\left\langle r, \nabla^{\prime} f \otimes \nabla^{\prime} h\right\rangle
$$

where

$$
\langle\nabla f(x), \xi\rangle \equiv \frac{d}{d t} f\left(e^{t \xi} x\right),\left\langle\nabla^{\prime} f(x), \xi\right\rangle \equiv \frac{d}{d t} f\left(x e^{t \xi}\right)
$$

are the left and right gradients respectively. The $\{,\}_{-}$Poisson bracket makes $G$ into a PLG. I will denote it simply by $\{$,$\} . The other bracket \{,\}_{+}$is also very important since it is non-degenerate almost everywhere and makes $G$ into a symplectic manifold. 
For every representation $\rho$ one can explicitly write the Poisson relations for the matrix elements of $T(x)=\rho(x)$ which are coordinates on the group as

$$
\left\{T_{1}, T_{2}\right\}=\left[r_{+}, T_{1} T_{2}\right]
$$

where $r_{+}=(\rho \otimes \rho) r$ and the subscript specifies the position in the tensor product. It is also useful to define $r_{-}=-(\rho \otimes \rho) \sigma(r)$.

The standard example of a factorizable PLG is $S L(N, C)$. In this case

$$
r=\frac{1}{2} \sum_{i, j=1}^{N-1}\left(A^{-1}\right)_{i j} H_{i} \otimes H_{j}+\sum_{i<j} E_{i j} \otimes E_{j i}
$$

where $A$ is the Cartan matrix, $H_{i}$ are Cartan generators and $E_{i j}$ are generators which in the fundamental representation are represented by matrices with only one non-vanishing entry equal to one in the $i j$ position. In this case we can give an explicit description of the dual group $S L(N, C)^{*}$ and its Poisson structure despite the fact that it is not quasi-triangular. Let $S L(N, C)^{*}$ be the group of pairs of upper and lower triangular matrices $\left\{\left(L^{+}, L^{-}\right)\right\}$where

$$
L^{+}=\left(\begin{array}{ccc}
a_{1} & & * \\
& \ddots & \\
0 & & a_{n}
\end{array}\right), L^{-}=\left(\begin{array}{ccc}
a_{1}^{-1} & & 0 \\
& \ddots & \\
* & & a_{n}^{-1}
\end{array}\right), \prod_{i=1}^{N} a_{i}=1 .
$$

The group multiplication is given by multiplying corresponding matrices within each pair. Using the same notation for matrix group elements and functions on the group, the Poisson brackets are:

$$
\left\{L_{1}^{+}, L_{2}^{+}\right\}=\left[r_{ \pm}, L_{1}^{+} L_{2}^{+}\right]
$$




$$
\begin{aligned}
& \left\{L_{1}^{-}, L_{2}^{-}\right\}=\left[r_{ \pm}, L_{1}^{-} L_{2}^{-}\right], \\
& \left\{L_{1}^{+}, L_{2}^{-}\right\}=\left[r_{+}, L_{1}^{+} L_{2}^{-}\right] .
\end{aligned}
$$

One can also define

$$
L=\left(L^{-}\right)^{-1} L^{+}
$$

and the Poisson brackets above become

$$
\left\{L_{1}, L_{2}\right\}=L_{1} r_{+} L_{2}+L_{2} r_{-} L_{1}-r_{+} L_{1} L_{2}-L_{1} L_{2} r_{-} .
$$

The derivation of this bracket from the quantum commutation relations is discussed in Appendix C. The map from $\left(L^{+}, L^{-}\right)$to $L$ is not one to one. It is a $2^{N-1}$ cover. Later we will define reality structures on this Poisson algebras.

Now I will give a more detailed description of the $S L(2, C)$ and $S L(2, C)^{*}$. Let

$$
T=\left(\begin{array}{ll}
a & b \\
c & d
\end{array}\right), \quad L=\left(\begin{array}{ll}
\alpha & \beta \\
\gamma & \delta
\end{array}\right) .
$$

The classical r-matrices can be written as $4 \times 4$ matrices

$$
r_{+}=\left(\begin{array}{cccc}
1 / 4 & 0 & 0 & 0 \\
0 & -1 / 4 & 1 & 0 \\
0 & 0 & -1 / 4 & 0 \\
0 & 0 & 0 & 1 / 4
\end{array}\right), r_{-}=\left(\begin{array}{cccc}
-1 / 4 & 0 & 0 & 0 \\
0 & 1 / 4 & 0 & 0 \\
0 & -1 & 1 / 4 & 0 \\
0 & 0 & 0 & -1 / 4
\end{array}\right) \text {. }
$$

Using (6.3) after some algebra one obtains

$$
\begin{aligned}
& \{a, b\}=a b / 2 \\
& \{a, c\}=a c / 2 \\
& \{a, d\}=c d
\end{aligned}
$$




$$
\begin{aligned}
& \{b, c\}=0 \\
& \{b, d\}=b d / 2, \\
& \{c, d\}=c d / 2 .
\end{aligned}
$$

Similarly using (6.6) one obtains

$$
\begin{aligned}
& \{\alpha, \beta\}=\alpha \beta \\
& \{\alpha, \gamma\}=-\alpha \gamma \\
& \{\alpha, \delta\}=0 \\
& \{\beta, \gamma\}=\alpha(\alpha-\delta), \\
& \{\beta, \delta\}=\alpha \beta, \\
& \{\gamma, \delta\}=-\alpha \gamma .
\end{aligned}
$$

A further decomposition of $L^{+}$as a diagonal matrix and an upper diagonal matrix with unit entries on the diagonal, and of $L^{-}$as a diagonal matrix and a lower diagonal matrix with unit entries on the diagonal, is possible. For the $S L(2, C)^{*}$ case, we have

$$
L^{+}=\left(\begin{array}{cc}
a & 0 \\
0 & a^{-1}
\end{array}\right)\left(\begin{array}{cc}
1 & \chi_{+} \\
0 & 1
\end{array}\right), L^{-}=\left(\begin{array}{cc}
a^{-1} & 0 \\
0 & a
\end{array}\right)\left(\begin{array}{rr}
1 & 0 \\
-\chi_{-} & 1
\end{array}\right) .
$$

It corresponds to Gauss's decomposition of $L$

$$
L=\left(\begin{array}{cc}
1 & 0 \\
\chi_{-} & 1
\end{array}\right)\left(\begin{array}{cc}
a^{2} & 0 \\
0 & a^{-2}
\end{array}\right)\left(\begin{array}{cc}
1 & \chi_{+} \\
0 & 1
\end{array}\right)
$$

To every LBA $\left(g, g^{*}\right)$ we can associate a factorizable LBA called the double Lie bialgebra $\left(d, d^{*}\right)$. First we define $d=g+g^{*}$, i.e. the direct sum of vector spaces. 
It has a natural bilinear form $\langle,\rangle_{d}$ defined in terms of the dual pairing $\langle$,$\rangle of g$ and $g^{*}$

$$
\langle(x, \xi),(y, \eta)\rangle_{d} \equiv\langle x, \eta\rangle+\langle y, \xi\rangle, x, y \in g, \xi, \eta \in g^{*}
$$

We define on $d$ the unique Lie algebra such that:

1. $g$ and $g^{*}$ are subalgebras;

2. the bilinear form $\langle,\rangle_{d}$ determined by the dual pairing is adjoint invariant.

On the basis of $d$ given by $\left\{e_{i}, e^{i}\right\}$, the commutator $[,]_{d}$ has the form

$$
\begin{aligned}
& {\left[e_{i}, e_{j}\right]_{d}=f_{i j}^{k} e_{k},} \\
& {\left[e^{i}, e^{j}\right]_{d}=f_{k}^{i j} e^{k}} \\
& {\left[e^{i}, e_{j}\right]_{d}=f_{i k}^{i} e^{k}-\tilde{f}_{i k}^{j} e_{k} .}
\end{aligned}
$$

Also $d^{*} \equiv g^{*} \oplus g$, i.e. it is the direct sum of Lie algebras $\left[e^{i}, e_{j}\right]_{d^{*}}=0$. The pair $\left(d, d^{*}\right)$ is a factorizable LBA with $r_{d} \equiv e^{i} \otimes e_{i} \in d \otimes d$, thus it is a projector on the $g$ factor. Note that $\operatorname{sl}(N, C)$ is almost the double of one of its Borel subalgebras ${ }^{1}$. We can exponentiate $d$ to a Lie group $D$ and $\{,\}_{-}$- will make it into a PGL.

The simplest example of the above structure is obtained if we start from the trivial LBA $\left(g, g^{*}\right)$, i.e. $g$ is a Lie algebra and $g^{*}$ its dual with the trivial commutator. $G$ is a Lie group with Lie algebra $g$ and $G^{*}=g^{*}$ is an abelian group. $D$ is the cotangent bundle $T^{*} G=G \times g^{*}$. The $\{,\}_{+}$bracket is the canonical Poisson

\footnotetext{
${ }^{1}$ It is the double of a Borel subalgebra divided by the Cartan subalgebra.
} 
bracket on the cotangent bundle, and $\{,\}_{-}$is the Lie bracket on $g^{*}$ extended by left translations to the cotangent bundle.

The double $D$ of a factorizable PLG $G$ can be described in more detail. $D$ is isomorphic with $G \times G$ as a group ${ }^{2}$. The groups $G$ and $G^{*}$ are subgroups of $D$ and are embedded as follows

$$
\begin{gathered}
G \subset G \times G, \quad T \rightarrow(T, T), \\
G^{*} \subset G \times G, \quad L \rightarrow\left(L^{+}, L^{-}\right) .
\end{gathered}
$$

Almost all elements $(x, y)$ of the double can be written in factorized form

$$
(x, y)=(T, T)^{-1}\left(L^{+}, L^{-}\right)=\left(\tilde{L}^{+}, \tilde{L}^{-}\right)^{-1}(\tilde{T}, \tilde{T})
$$

A pair of Poisson manifolds $\left(P, P^{\prime}\right)$ is called a dual pair $[51,71]$ if there exists a symplectic manifold $S$ and two projections $\pi$ and $\pi^{\prime}$

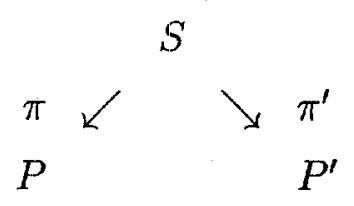

such that the sets of functions which are pullbacks of functions on $P$ and $P^{\prime}$ centralize each other

$$
\left\{\pi^{*}(f), \pi^{*}\left(f^{\prime}\right)\right\}_{S}=0
$$

An important theorem $[51,70]$ states that each symplectic leaf of $P$ is obtained by projecting on $P$ the preimage of an element $a$ of $P^{\prime}$

$$
\pi\left(\pi^{\prime-1}(a)\right), \quad a \in P^{\prime}
$$

\footnotetext{
${ }^{2}$ This is only true for complex groups. If $G$ has a reality structure the double is obtain by imposing a reality structure on $G^{c} \times G^{c}$ where $G^{c}$ is the complexification of $G$.
} 
The manifolds $D / G$ and $G \backslash D$ form a dual pair. The symplectic manifold is the double $D$ of $G$ with the $\{,\}_{+}$bracket. The following projections

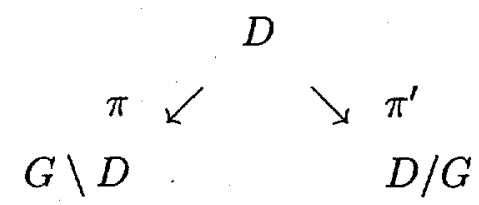

can be used to induce Poisson structures on $D / G$ and $G \backslash D$. Since $D$ is factorizable $G^{*} \cong G \backslash D$. Moreover the Poisson structure induced on $G \backslash D$ from $D$ coincides with the original Poisson structure on $G^{*}$. Then the above theorem gives the symplectic leaves of $G^{*}$. In particular if $G$ is factorizable, $\pi^{\prime}(x, y) \equiv x y^{-1}=a$ and the preimage of $a$ has elements of the form $(a y, y)$. Then $\pi(x, y)=y^{-1} x=y^{-1} a y$, thus the symplectic leaves are given by the orbits of the coadjoint action of $G$ on $G \backslash D$. This action is also known as the dressing action [70] .

$$
G \times(G \backslash D) \rightarrow G \backslash D,(y, a) \rightarrow y^{-1} a y .
$$

\subsection{Symplectic Leaves}

In the first part of this section, I will discuss the $S L(N, C)$ case. So far, everything was complex. The simplest reality structure one can impose is to require everything to be real. We then obtain $S L(N, R)$, its double, dual etc. However, we want to obtain $S U(N)$. We start on the double with the reality structure

$$
x^{\dagger}=y^{-1} \text {. }
$$


Since $G$ and $G^{*}$ are subgroups, this induces the following reality structures

$$
T^{\dagger}=T^{-1},\left(L^{+}\right)^{\dagger}=\left(L^{-}\right)^{-1}
$$

Once we impose (6.10) the dual group is no longer simply connected, since $a_{i}$ in (6.4) are real and non-zero. Define $S U(N)^{*}$ as the component connected to the unit element of the group.

$$
S U(N)^{*}=\left\{\left(L^{+}, L^{-}\right) \in S L^{*}(N, C) \mid\left(L^{+}\right)^{\dagger}=\left(L^{-}\right)^{-1}, a_{i}>0\right\} .
$$

We can also describe $S U(N)^{*}$ in terms of $L$ as the set of hermitian, positive definite matrices of determinant one. Then the map $\left(L^{+}, L^{-}\right) \rightarrow L=\left(L^{-}\right)^{-1} L^{+}$is one to one and the factorization is unique.

For $S U(2)^{*}$ the reality structure is $\bar{\alpha}=\alpha, \bar{\delta}=\delta, \bar{\beta}=\gamma$.

To summarize, the double of $S U(N)$ is $S L(N, C)$, and the factorization (6.9) can be written $x=T^{-1} L^{+}$, that is to say, any matrix of determinant one can be decomposed uniquely as the product of a special unitary matrix and an upper triangular matrix with real positive diagonal entries ${ }^{3}$.

In particular the double of $S U(2)$ is the proper Lorentz group $S L(2, C)$. It is interesting to note that the double of the trivial PLG $S U(2)$, i.e. its cotangent bundle, is the proper homogeneous Galilean group.

Using the two factorizations

$$
(x, y)=\left(T^{-1} L^{+}, T^{-1} L^{-}\right)=\left(\left(\tilde{L}^{+}\right)^{-1} \tilde{T},\left(\tilde{L}^{-}\right)^{-1} \tilde{T}\right)
$$

\footnotetext{
${ }^{3}$ Note that $y$ is not independent $y=\left(x^{\dagger}\right)^{-1}$
} 
and the projections $\pi(x, y)=y^{-1} x, \pi^{\prime}(x, y)=x y^{-1}$ we obtain the following form for the symplectic leaves

$$
\pi\left(\pi^{\prime-1}\left(\left(\tilde{L}^{+}\right)^{-1} \tilde{L}^{-}\right)\right)=\left\{\left(L^{-}\right)^{-1} L^{+}=(\tilde{T})^{-1} \tilde{L}^{-}\left(\tilde{L}^{+}\right)^{-1} \tilde{T} \mid \tilde{T} \in S U(2)\right\}
$$

where $\left(\tilde{L}^{+}, \tilde{L}^{-}\right) \in S U(2)^{*}$ is fixed, and $\tilde{T}$ parametrizes the leave. This is just the orbit of the right Poisson coadjoint action of $S U(2)$ on $S U(2)^{*}$

$$
L \rightarrow T^{-1} L T
$$

It is convenient to use an exponential parametrization of $L=\left(L^{-}\right)^{-1} L^{+}$

$$
L=\exp \left(x_{i} \sigma_{i}\right)=\cosh (r)+\sinh (r)\left(\begin{array}{cc}
n_{3} & n_{-} \\
n_{+} & -n_{3}
\end{array}\right)
$$

where $\sigma_{i}$ 's are the Pauli matrices, $r^{2}=\sum_{i} x_{i}^{2}$ and $n_{i}=x_{i} / r$. Since $\operatorname{tr}(L)=$ $2 \cosh (r)$ is invariant under the coadjoint action we see that the symplectic leaves are spheres of radius $r$ except for the $r=0$ leaf, which is zero dimensional. In terms of the exponential parametrization, the Poisson algebra (6.8) becomes

$$
\begin{aligned}
& \left\{x_{ \pm}, x_{3}\right\}= \pm x_{ \pm}\left(x_{3}+r \operatorname{coth}(r)\right), \\
& \left\{x_{-}, x_{+}\right\}=2 x_{3}\left(x_{3}+r \operatorname{coth}(r)\right) .
\end{aligned}
$$

Since $r$ is constant on symplectic leaves it must be central in the above Poisson algebra, which can be checked by direct computation. These Poisson spheres and their quantization were first studied in [57]. One can parametrize the radius $r$ sphere using stereographic projection coordinates $z, \bar{z}$

$$
z=\frac{x_{-}}{r-x_{3}}, \bar{z}=\frac{x_{+}}{r-x_{3}} .
$$


After some straightforward algebra we obtain

$$
\{\bar{z}, z\}_{r}=\frac{1}{2}(1+z \bar{z})^{2}\left(\frac{z \bar{z}-1}{z \bar{z}+1}+\operatorname{coth}(r)\right) .
$$

The right action of $S U(2)$ on $z$ by fractional transformations

$$
z^{\prime}=\frac{\bar{a} z-b}{\bar{b} z+a}
$$

is a Poisson action i.e. $a, b, c, d$ have non-trivial bracket given by (6.7). Since our path integral is formulated in real time, we do a Wick rotation and obtain the Minkowski Poisson bracket

$$
\{\bar{z}, z\}_{r}=\frac{i}{2}(1+z \bar{z})^{2}\left(\frac{z \bar{z}-1}{z \bar{z}+1}+\operatorname{coth}(r)\right)
$$

differing from the original one by a phase factor.

Using non-singular coordinates around the south pole $w=-1 / z$ the Poisson bracket becomes

$$
\{\bar{w}, w\}_{r}=\frac{i}{2}(1+w \bar{w})^{2}\left(-\frac{w \bar{w}-1}{w \bar{w}+1}+\operatorname{coth}(r)\right)
$$

thus the Poisson structure is not north-south symmetric. The infinite $r$ limit is singular at the south pole. This particular Poisson structure and its quantization was studied in $[18,19]$.

The small $r$ limit is dominated by the $\operatorname{coth}(r)$ term and

$$
\{\bar{z}, z\}_{r} \approx \frac{i}{2} \operatorname{coth}(r)(1+z \bar{z})^{2} .
$$

This is the standard Poisson bracket on a sphere of radius $\operatorname{coth}^{1 / 2}(r)$. The right action by fractional transformations on (6.12) leaves this Poisson bracket invariant. Thus the small radius symplectic leaves are almost rotationally invariant. 
Next we obtain the symplectic form on the leaves. Let $f, h$ be functions on the leaf; each $f$ defines a vector field $v_{f}$ such that $v_{f}(h)=\{f, h\}$. Then the symplectic form is defined by

$$
\Omega\left(v_{f}, v_{h}\right) \equiv\{h, f\}
$$

In local coordinates, the Poisson bracket and the symplectic form have the form

$$
\{f, h\}=P^{i j} \partial_{i} f \partial_{j} h, \Omega=\frac{1}{2} \Omega_{i j} d x^{i} \wedge d x^{j}
$$

and the two antisymmetric tensors satisfy

$$
P^{i j} \Omega_{j k}=\delta_{k}^{i}
$$

In complex coordinates, this is simply $P^{\bar{z} z} \Omega_{z \bar{z}}=1$, and gives

$$
\Omega=-\frac{2}{i} \frac{\bar{d} z \wedge d z}{(1+z \bar{z})^{2}}\left(\frac{z \bar{z}-1}{z \bar{z}+1}+\operatorname{coth}(r)\right)^{-1}=-\frac{\Omega_{0}}{n_{3}+\operatorname{coth}(r)}
$$

where $\Omega_{0}$ is the standard area 2 -form on the unit sphere.

\subsection{Path Integral Quantization}

The path integral quantization of the Poisson algebra on the leaves of $s u(2)^{*}$ was discussed in $[53,1]$. Quantization of these leaves gives the unitary representations of $S U(2)$. We will do the same for the symplectic leaves above and obtain the unitary representations of $\mathcal{U}_{q}(s u(2))$ algebra. This is in fact a Hopf algebra but we concentrate here on the algebra structure ${ }^{4}$.

\footnotetext{
${ }^{4}$ The coproduct and antipode of the $L^{ \pm}$generators are the same as in the classical PoissonHopf algebra
} 
Before starting the quantization we have to find canonical coordinates on the leaves. Note that

$$
\Omega_{0}=\sin \theta d \theta \wedge d \phi=d(-\cos (\theta)) \wedge d \phi
$$

thus $(-\cos (\theta), \phi)$ are Darboux coordinates on the standard $S_{2}$. Similarly

$$
\Omega=d\left[-\ln \left(n_{3}+\operatorname{coth}(r)\right)\right] \wedge d \phi
$$

so we define

$$
J \equiv-\ln \left[\frac{n_{3}+\operatorname{coth}(r)}{\left(\operatorname{coth}^{2}(r)-1\right)^{1 / 2}}\right]=-\ln \left[\cosh (r)+\sinh (r) n_{3}\right]
$$

where the denominator was fixed by the requirement that $J$ spans a symmetric interval $(-r, r)$. We have $\Omega=d J \wedge d \phi=d(J d \phi)$ so we define the Poincare 1-form $\Theta$

$$
\Theta=J d \phi+c d \phi
$$

where $c$ is a constant to be fixed later. Thus the Poisson sphere of radius $r$ is parametrized by $J$ and $\phi$ as

$$
n_{3}=\sinh ^{-1}(r)\left(e^{-J}-\cosh (r)\right), n_{ \pm}=\left(1-n_{3}^{2}\right)^{1 / 2} e^{\mp i \phi} .
$$

The Poisson algebra on any leaf can be quantized, but in general these quantum algebras will not have unitary representations. Unitarity leads to a quantization of the radius of the Poisson sphere. Before starting the Poisson case let us review two different quantization conditions used in $[53,1]$ for the trivial case. In [1] a geometric quantization condition similar to that used for the Dirac monopole or the 
Wess-Zumino-Witten model was used to obtain the allowed values of the radius. The action must be continuous as the path crosses over the poles. Equivalently

$$
e^{i / \hbar \oint \Theta}=1
$$

where the integral is over an infinitesimal loop around the poles. However this condition was only used to determine the characters of the representations. Also note that, unlike the Dirac monopole where the action is a configuration space action, both in the trivial and the Poisson case one has a phase space action.

However in [53] it was shown that in order to obtain the matrix elements of $s u(2)$ a non-trivial phase has to exist as the path crosses the poles. Requiring the correct matrix elements one obtains the quantization condition

$$
e^{i / \hbar \oint \Theta}=-1
$$

This gives the same result as (6.13) for the Cartan generator and thus for the characters. Here I will use (6.14) and show that we obtain the standard matrix elements of the quantum group generators.

Imposing (6.14) at the north and south poles we obtain the quantization $r=$ $N \hbar / 2$ where $N$ is a positive integer. For $N$ odd one can set $c=0$ but a non-zero $c$ is required for even $N$. The simplest choice is $c=\hbar / 2$. We can write the two cases together as

$$
\Theta=(J+M \hbar / 2) d \phi, M=0,1 .
$$

Next I list some of the functions on the Poisson sphere that I will quantize, 
expressed in terms of Darboux variables $J, \phi$

$$
\begin{aligned}
\alpha & =e^{-J} \\
\beta & =\left(-1+2 \cosh (r) e^{-J}-e^{-2 J}\right)^{1 / 2} e^{i \phi} \\
\gamma & =\left(-1+2 \cosh (r) e^{-J}-e^{-2 J}\right)^{1 / 2} e^{-i \phi} \\
\delta & =2 \cosh (r)-e^{-J} \\
a & =e^{-J / 2} \\
\chi_{ \pm} & =\left(-1+2 \cosh (r) e^{J}-e^{2 J}\right)^{1 / 2} e^{ \pm i \phi}
\end{aligned}
$$

The general structure of this functions is

$$
\mathcal{O}(J, \phi)=\mathcal{F}(J) e^{i p \phi}, p=0, \pm 1
$$

Note also that

$$
\operatorname{tr}(L)=2 \cosh (r)=2 \cosh (N \hbar / 2)=q^{N}+q^{-N},
$$

where we introduced $q \equiv e^{\hbar / 2}$. Since $\operatorname{tr}(L)$ only depends on $r$, it is central in the Poisson algebra and will be central in the quantum algebra. In fact $\operatorname{tr}(L)$ is the Casimir of $\mathcal{U}_{q}(s u(2))$.

Next we discuss the Feynman path integral. Consider first for simplicity a Hamiltonian $H(J)$, i.e. a function of $J$ and not of $\phi$. Wave functions are functions on $S_{1}$ (or periodic functions of $\phi$ ) and let $|\phi\rangle$ be a $\phi$ eigenvector. The propagator on $S_{1}$ can be expressed in terms of the propagator on the covering space of $S_{1}$, which is the real line by

$$
\left\langle\phi^{\prime}\left|e^{-\frac{i}{\hbar} H T}\right| \phi\right\rangle=\sum_{n \in Z}\left\langle\phi^{\prime}+2 \pi n\left|e^{-\frac{i}{\hbar} H T}\right| \phi\right\rangle_{0}
$$


where formally

$$
\left\langle\phi^{\prime}\left|e^{-\frac{i}{\hbar} H T}\right| \phi\right\rangle_{0}=\iint \frac{\mathcal{D} J \mathcal{D} \phi}{2 \pi \hbar} e^{\frac{i}{\hbar} \int_{0}^{T}[\Theta-H(J) d t]}
$$

where $\phi$ is integrated over the whole real line and $J$ over the $(-r, r)$ interval. To make sense of the formal expression we divide $T$ into $P$ intervals and let $\phi_{0}=\phi, \phi_{P}=\phi^{\prime}$. Then

$$
\left\langle\phi^{\prime}\left|e^{-\frac{i}{\hbar} H T}\right| \phi\right\rangle_{0}=\int \frac{\prod_{i} d J_{i}}{2 \pi \hbar} \int \prod_{i} d \phi_{i} e^{i / \hbar \sum_{i}\left[\left(J_{i}+c\right)\left(\phi_{i}-\phi_{i-1}\right)-H\left(J_{i}\right) T / P\right]}
$$

The $\phi$ integration can be performed leading to delta functions which allow us to do all but one of the $J$ integrals. Then the propagator on $S_{1}$ takes the form

$$
\left\langle\phi^{\prime}\left|e^{-\frac{i}{\hbar} H T}\right| \phi\right\rangle=\sum_{n \in Z} \int_{-N \hbar / 2}^{N \hbar / 2} \frac{d J}{2 \pi \hbar} e^{-i / \hbar H(J) T} e^{i / \hbar(J+c)\left(\phi^{\prime}+2 \pi n\right)} e^{-i / \hbar(J+c) \phi}
$$

Using the Poisson resummation formula

$$
\sum_{n \in Z} e^{2 \pi i n \alpha}=\sum_{k \in Z} \delta(\alpha-k)
$$

we perform the last integral and obtain

$$
\left\langle\phi^{\prime}\left|e^{-\frac{i}{\hbar} H T}\right| \phi\right\rangle=\sum_{\substack{k \\\left|J_{k}\right| \leq N \hbar / 2}} \frac{e^{i k \phi^{\prime}}}{\sqrt{2 \pi}} e^{-i / \hbar H\left(J_{k}\right) T} \frac{e^{-i k \phi}}{\sqrt{2 \pi}}
$$

where $J_{k}=\hbar(k-M / 2)$. The sum is over all integers $k$ such that $(-N+M) / 2 \leq$ $k \leq(N+M) / 2$. We see that not all states propagate. We can make the path integral unitary by projecting out the states that do not propagate. Define the Hilbert space as the vector space spanned by the vectors

$$
|m\rangle=\int \frac{d \phi}{\sqrt{2 \pi}} e^{i(m+M / 2) \phi}|\phi\rangle, \quad m=-j, \ldots, j
$$




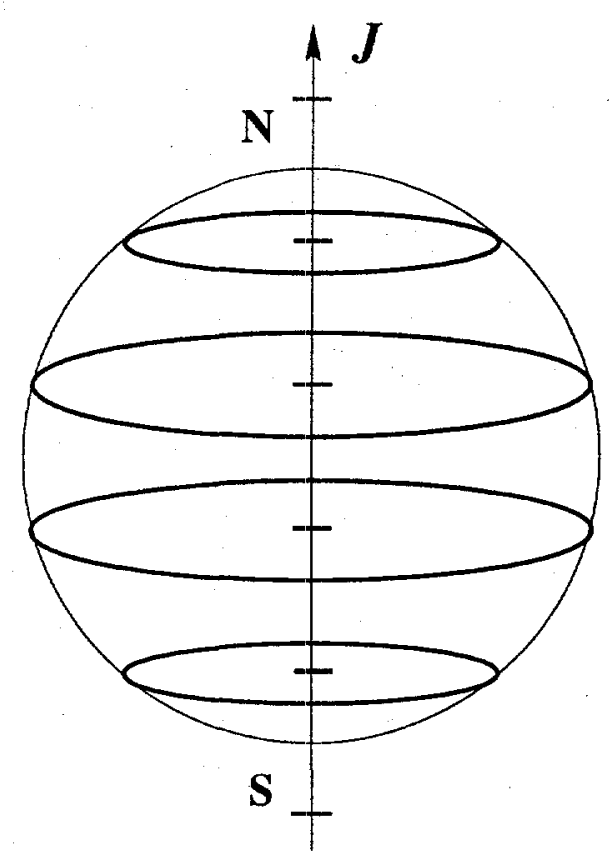

Figure 6.1: The allowed values of $J$ do not coincide with the North or South poles.

where, according to angular momentum conventions, $j$ is a half integer such that $N=2 j+1$. Note that the exponent is always an integer and $N$ is the total number of states. As depicted in figure 6.1 the maximum value $J= \pm N \hbar / 2$ is not reached quantum mechanically. It differs from the results in [1] but agrees with [53] as previously mentioned. It was pointed out in [53] that this is similar to the non-zero ground state energy of the harmonic oscillator.

\subsection{Matrix Elements and the Quantum Algebra}

Since this is a phase space path integral some care must be taken when quantizing functions which depend on canonically conjugate variables. The standard mid-point prescription for a function of the form $\mathcal{J}(J) \Phi(\phi)$ is to write it 
as $\mathcal{J}\left(J_{i}\right) \Phi\left[\left(\phi_{i}+\phi_{i-1}\right) / 2\right]$ in the path integral. Thus for functions of the form $\mathcal{O}(J, \phi)=\mathcal{F}(J) e^{i p \phi}$ I will use $\mathcal{F}\left(J_{i}\right) e^{i p\left(\phi_{i}+\phi_{i-1}\right) / 2}$. To calculate the matrix elements of such an operator we insert it in the path integral (6.18) with $H=0$ and take $T$ infinitesimal. For the prescription above it is sufficient to consider only one time interval. The matrix elements are

$$
\begin{gathered}
\left\langle\phi^{\prime}|\mathcal{O}| \phi\right\rangle=\sum_{n \in Z} \int \frac{d J}{2 \pi \hbar} e^{i / \hbar(J+c)\left(\phi^{\prime}+2 \pi n-\phi\right)} \mathcal{F}(J) e^{i p\left(\phi^{\prime}+2 \pi n+\phi\right) / 2}= \\
\sum_{k} \frac{e^{i k \phi^{\prime}}}{\sqrt{2 \pi}} \mathcal{F}\left(J_{k}\right) \frac{e^{-i(k-p) \phi}}{\sqrt{2 \pi}}
\end{gathered}
$$

where $J_{k}=\hbar(k-M / 2-p / 2)$, and I used Poisson resummation before performing the $J$ integral. Then the matrix elements in the $\{|m\rangle\}$ basis are given by

$$
(\mathcal{O})_{m^{\prime} m}=\left\langle m^{\prime}|\mathcal{O}| m\right\rangle=\mathcal{F}\left[\left(m^{\prime}-p / 2\right) \hbar\right] \delta_{m^{\prime}-p-m, 0}, m=-j, \ldots, j
$$

Using the opposite mid-point prescription $\mathcal{F}\left[\left(J_{i}+J_{i-1}\right) / 2\right] e^{i p \phi_{i}}$ gives the same matrix elements. However in this case one has to consider at least two time intervals if working in the $\phi$ representation. This prescription is more convenient when working in the $J$ representation.

We can use (6.19) to calculate matrix elements of any function on $S U(2)^{*}$. Mid-point prescription in the path integral results in a special ordering of the quantum operators, when expressed in terms of $J$ and $\phi$, called Weyl ordering. If one starts from the Gauss's decomposition, uses path integral to obtain the matrix elements of $a$ and $\chi_{ \pm}$and then uses them to express $L^{ \pm}$as products of quantum matrices, we obtain the quantum commutation relations [30]. Using (6.19) we 
obtain

$$
\begin{aligned}
(a)_{m^{\prime} m} & =e^{-\hbar m^{\prime} / 2} \delta_{m^{\prime}-m, 0} \\
\left(\chi_{ \pm}\right)_{m^{\prime} m} & =\left(-1+2 \cosh (\hbar(j+1 / 2)) e^{\hbar\left(m^{\prime} \mp 1 / 2\right)}-e^{2 \hbar\left(m^{\prime} \mp 1 / 2\right)}\right)^{1 / 2} \delta_{m^{\prime}-m \mp 1,0}
\end{aligned}
$$

One can check by direct calculation that relations (6.20) are representations of the algebra generated by $a, \chi_{ \pm}$with relations

$$
\begin{aligned}
\chi_{+} a & =q a \chi_{+} \\
\chi_{-} a & =q^{-1} a \chi_{-} \\
q \chi_{+} \chi_{-}-q^{-1} \chi_{-} \chi_{+} & =\lambda\left(a^{-4}-1\right)
\end{aligned}
$$

where $\lambda \equiv q-q^{-1}$. Using this we define the quantum matrices $L^{ \pm}$as

$$
\begin{aligned}
& L^{+}=\left(\begin{array}{cc}
a & 0 \\
0 & a^{-1}
\end{array}\right)\left(\begin{array}{cc}
1 & \chi_{+} \\
0 & 1
\end{array}\right), \\
& L^{-}=\left(\begin{array}{cc}
a^{-1} & 0 \\
0 & a
\end{array}\right)\left(\begin{array}{cc}
1 & 0 \\
-\chi_{-} & 1
\end{array}\right) .
\end{aligned}
$$

One can use (6.21) to check that $L^{ \pm}$satisfies the quantum group commutations relations $[28,30,88]$

$$
\begin{aligned}
& R_{ \pm} L_{1}^{ \pm} L_{2}^{ \pm}=L_{2}^{ \pm} L_{1}^{ \pm} R_{ \pm} \\
& R_{+} L_{1}^{+} L_{2}^{-}=L_{2}^{-} L_{1}^{+} R_{+} \\
& R_{-} L_{1}^{-} L_{2}^{+}=L_{2}^{+} L_{1}^{-} R_{-}
\end{aligned}
$$

where the quantum matrices are given in the Appendix C. Alternatively, using the representations

$$
L^{+}=\left(\begin{array}{cc}
q^{-H / 2} & q^{-1 / 2} \lambda X_{+} \\
0 & q^{H / 2}
\end{array}\right), L^{-}=\left(\begin{array}{cc}
q^{H / 2} & 0 \\
-q^{1 / 2} \lambda X_{-} & q^{-H / 2}
\end{array}\right)
$$


of the quantum $L^{ \pm}$in terms of Jimbo-Drinfeld generators discussed in Appendix C, the relations (6.21) are equivalent to

$$
\left[H, X_{ \pm}\right]= \pm 2 X_{ \pm},\left[X_{+}, X_{-}\right]=\frac{q^{H}-q^{-H}}{q-q^{-1}}
$$

The Jimbo-Drinfeld generators of $\mathcal{U}_{q}(s u(2))$ can be obtained in the path integral by inserting

$$
\begin{aligned}
H & =\hbar^{-1} 2 J \\
X_{ \pm} & =\lambda^{-1}\left[2(\cosh (r)-\cosh (J)]^{1 / 2} e^{ \pm i \phi}\right.
\end{aligned}
$$

Note that unlike $a$ and $\chi_{ \pm}$the insertions above are already quantum. In addition while the functional dependence in terms of $J$ and $\phi$ can be easily obtained from (6.23), the overall normalization of $X_{ \pm}$has been adjusted to give the standard result. The same kind of normalization adjustments are necessary if one tries to insert the matrix elements of $L^{ \pm}$directly into the path integral. This just reflects ordering ambiguities of quantum operators. Alternatively one could get the standard result without any adjustments of normalization by using a nonmidpoint prescription. For example the off-diagonal element of $L^{+}$equals $a \chi_{+}$ with this specific ordering in the quantum case. Since the path integral gives time ordering we can obtain the desired quantum ordering by using the following prescription

$$
e^{-J_{i} / 2}\left(-1+2 \cosh (r) e^{\left(J_{i}+J_{i-1}\right) / 2}-e^{J_{i}+J_{i-1}}\right)^{1 / 2} e^{+i \phi}
$$

Note that I only used a mid-point prescription for $\chi_{+}$and not for $a$. The matrix 
elements obtained using (6.19) are

$$
\begin{aligned}
(H)_{m^{\prime} m} & =2 m \delta_{m^{\prime}-m, 0} \\
\left(X_{ \pm}\right)_{m^{\prime} m} & =\{2 \operatorname{coth}[\hbar(j+1 / 2)]-2 \operatorname{coth}[\hbar(m \pm 1 / 2)]\}^{1 / 2} \delta_{m^{\prime}-m \mp 1,0}
\end{aligned}
$$

The generators of $s u(2)$ are obtained using

$$
\begin{aligned}
\tilde{H} & =2 J \\
\tilde{X}_{ \pm} & =\left(r^{2}-J^{2}\right)^{1 / 2} e^{ \pm i \phi}
\end{aligned}
$$

In this case it is possible to write all generators without using $\hbar$ while in the deformed case a different rescaling for each generator is required to eliminate $\hbar$. The matrix elements obtained using (6.19)

$$
\begin{aligned}
(\tilde{H})_{m^{\prime} m} & =2 \hbar m \delta_{m^{\prime}-m, 0} \\
\left(\tilde{X}_{ \pm}\right)_{m^{\prime} m} & =\hbar\left[(j+1 / 2)^{2}-(m \pm 1 / 2)^{2}\right]^{1 / 2} \delta_{m^{\prime}-m \mp 1,0}
\end{aligned}
$$

are just the standard matrix elements of the $s u(2)$ algebra

$$
\left[\tilde{H}, \tilde{X}_{ \pm}\right]= \pm 2 \hbar \tilde{X}_{ \pm},\left[\tilde{X}_{+}, \tilde{X}_{-}\right]=\hbar \tilde{H}
$$

\subsection{Remarks}

In addition to trying to generalize the results in $[53,1]$ my goal in this chapter was to better understand the quantization (6.22) of the Poisson bracket (6.5). Any $R_{ \pm}$satisfying $R_{ \pm}=1+\hbar r_{ \pm}+\mathcal{O}\left(\hbar^{2}\right)$ used in (6.22) would give the same Poisson bracket in the classical limit. The $\mathcal{O}\left(\hbar^{2}\right)$ and higher order terms are fixed by 
requiring that (6.22) are commutation relations of a Hopf algebra deformation of the original Poisson-Hopf algebra. It is natural then to ask what is the relation of this quantization to the quantization known as Weyl quantization. Of course this question could be answered using algebraic methods without appealing to path integrals. At least for the case of $S U(2)$, I found that the functions $\chi_{ \pm}$and $a$ appearing in the Gauss's decomposition play a special role. Their quantization using Weyl ordering gives the same commutation relations as in the quantum group quantization. It would be interesting to investigate if this result still holds for an arbitrary $S U(N)$.

It should be possible to generalize the path integral to arbitrary classical groups. The similarity between the trivial and the Poisson cases for $S U(2)$ suggests that a starting point could be the path integral quantization of the coadjoint orbits of classical groups discussed in [1].

The existence of a non-trivial phase as the path crosses the poles discussed in [53] is present in the Poisson case too. The origin of this phase can be explained as follows. The coordinates $\phi$ and $J$ are singular at the two poles. To analyze what happens as the trajectory passes over one of the poles one should introduce canonical variables which are regular there. In the new coordinates the action should vary smoothly as the trajectory crosses the pole. This would imply that in the $\phi$ and $J$ coordinates the action would jump only if the change of variables in the path integral has a nontrivial Jacobian. Formally one does not expect this to happen since the change of variables is a canonical transformation. However if 
one regularizes the path integral by introducing for example a time lattice, and carefully analyzes the change of variables, a nontrivial Jacobian is indeed found.

Let us now compare the trivial and Poisson cases. The symplectic leaves in both cases are spheres parametrized by $(z, \bar{z})$ in stereographic projection. The group $S U(2)$ acts in the same way on the leaves in the two cases, i.e. by standard rotations of the spheres, but in the trivial case the bracket is invariant under the action, while in the Poisson case the action is only a Poisson action. However, once the symplectic form is expressed in Darboux coordinates $(J, \phi)$ the leaves appear to be identical. As a consequence the path integral has the same form as in $[53,1]$, but since the transformation to the Darboux variables is non-trivial in the Poisson case, $S U(2)$ acts in a complicated way on the leaves, and functions on $S U(2)^{*}$ have a complicated dependence on $(J, \phi)$. Compare for example (6.25) and (6.26). Thus the same path integral generates different matrix elements because we insert different functions in the trivial and Poisson cases. This shows explicitly that on the same symplectic manifold one can implement both a trivial and a Poisson symmetry. The question of which is the actual symmetry of the system is a dynamical one, and can only be answered after we know the Hamiltonian. Finally, I conjecture that as in the $S U(2)$ case, for an arbitrary classical group, the path integral has the same form in the trivial and Poisson cases. 


\section{Chapter 7}

\section{Quantum Algebra of the Particle Moving on the $q$-Deformed Mass-Hyperboloid}

In this chapter I will introduce a reality structure inspired by the noncompact WZNW-model. In Section 7.1, contains a short review of the quantum algebra $\mathrm{T}^{*} G_{q}$. I discuss the commutation relations for operators generating both left and right translations, since both forms are necessary to define or to check the involutions presented in the next sections. Section 7.2 briefly covers the reality structure of [5]. In section 7.3 , I present the main result of this chapter, a reality structure corresponding to a generalized mass-hyperboloid configuration space and its associated $q$-deformed phase space. In Section 7.4, I consider the simple quantum mechanical system of [5] and show its compatibility with the *-structure introduced in the previous section. In the last Section I present some evidence for the relevance of this reality structure to the non-compact WZNW-model.

\subsection{Review of the Algebra on $\mathrm{T}^{*} G_{q}$}

In this section I present a brief review of the defining relations of the $q$-deformed cotangent bundle [3] also known as the Heisenberg double or as the smash prod- 
uct $[88,89]$. The main purpose of this section is to fix the notation. I will follow closely the presentation in [5] where a more detailed exposition can be found.

Let $G$ be the Lie group $S L(N, \mathrm{C})$, and sometimes for simplicity I will take $G=S L(2, \mathrm{C})$. Most of the content of this chapter can be easily extended to arbitrary classical groups. Now consider the quantum $R_{+}$matrix associated to the Lie group $G$. This is a matrix depending on a parameter $q$ and acting in the tensor product of two fundamental representations. For example the $R_{+}$of $S L(2, \mathrm{C})$ is the following $4 \times 4$ matrix

$$
R_{+}=q^{-1 / 2}\left(\begin{array}{cccc}
q & 0 & 0 & 0 \\
0 & 1 & q-q^{-1} & 0 \\
0 & 0 & 1 & 0 \\
0 & 0 & 0 & q
\end{array}\right) .
$$

It is convenient to also use the $R_{-}$matrix defined as

$$
R_{-}=P R_{+}^{-1} P
$$

where $P$ is the permutation operator in the tensor space of the two fundamental representations

$$
P(a \otimes b)=b \otimes a
$$

Next I will define the quantum algebra $\mathrm{T}^{*} G_{q}$, the quantum deformation of the cotangent bundle. Let $g$ and $\Omega_{ \pm}$be matrices acting in the fundamental representation of $G$. The $\Omega_{ \pm}$matrices are upper and lower triangular matrices. In addition the diagonal elements of $\Omega_{+}$equal those of $\Omega_{-}^{-1} . \mathrm{T}^{*} G_{q}$ is the algebra generated by $g$ and $\Omega_{ \pm}$and satisfying the following set of relations divided for convenience into 
three groups

$$
\begin{array}{r}
R_{ \pm} g^{1} g^{2}=g^{2} g^{1} R_{ \pm} \\
R_{ \pm} \Omega_{+}^{1} \Omega_{+}^{2}=\Omega_{+}^{2} \Omega_{+}^{1} R_{ \pm} \\
R_{ \pm} \Omega_{-}^{1} \Omega_{-}^{2}=\Omega_{-}^{2} \Omega_{-}^{1} R_{ \pm} \\
R_{+} \Omega_{-}^{1} \Omega_{-}^{2}=\Omega_{-}^{2} \Omega_{+}^{1} R_{+} \\
R_{-} \Omega_{-}^{1} \Omega_{+}^{2}=\Omega_{+}^{2} \Omega_{-}^{1} R_{-} \\
R_{+} \Omega_{+}^{1} g^{2}=g^{2} \Omega_{+}^{1} \\
R_{-} \Omega_{-}^{1} g^{2}=g^{2} \Omega_{-}^{1} .
\end{array}
$$

All the above relations are operator matrices acting in the tensor product of two fundamentals, and the superscript indicates on which factor the respective matrix acts. The $R$ matrices without any superscript act in both spaces. One can show that the quantum determinant of the matrices $g$ and $\Omega_{ \pm}$is central and can be set equal to one

$$
\operatorname{det}_{q}(g)=\operatorname{det}_{q}\left(\Omega_{ \pm}\right)=1
$$

For the $S L(N, \mathrm{C})$ groups these are all the relations, while for the other classical groups additional relations, for example orthogonality relations, have to be imposed. Note also that, unlike (7.2)(7.3), the relation (7.4) is not homogeneous in $R_{ \pm}$thus the normalization of $R_{ \pm}$is important.

The above relations are not independent. For example the $R_{-}$relations can 
be obtained from the $R_{+}$relations using (7.1) and

$$
X^{2}=P X^{1} P
$$

The subalgebra generated by the matrix elements of $g$ with relations (7.2) is in fact a Hopf algebra denoted $\operatorname{Fun}_{q}(G)$ and represents a deformation of the Hopf algebra of function on the $G$ Lie group [30]. Also, the subalgebra generated by $\Omega_{ \pm}$ with relations (7.3) is a quasitriangular Hopf algebra called the quantum universal enveloping algebra $[28,45,30]$, and is denoted $U_{q}(\mathfrak{g})$ where the $\mathfrak{g}$ in the brackets is the Lie algebra of the Lie group $G$. For example the coproduct of $\operatorname{Fun}_{q}(G)$ on the matrix elements of $g$ is given by

$$
\triangle(g)=g \dot{\otimes} g
$$

where the dot means multiplication in matrix space. Similarly the coproduct in $U_{q}(\mathfrak{g})$ on the matrix elements $\Omega_{ \pm}$reads

$$
\triangle\left(\Omega_{ \pm}\right)=\Omega_{ \pm} \dot{\otimes} \Omega_{ \pm}
$$

On the other hand $\mathrm{T}^{*} G_{q}$ is not a Hopf algebra. We emphasize this, since there is a related algebra, the Drinfeld double, which has the same generators but different mixed relations and is a Hopf algebra.

The mixed relations (7.4) describe how to combine the above subalgebras into the larger algebra $\mathrm{T}^{*} G_{\boldsymbol{q}}$. They appear as commutation relations in $[3,88,89]$ but in an abstract form as the pairing of dual Hopf algebras they were already present in $[30]$. 
One can relate the $\Omega_{ \pm}$with the more traditional Drinfeld-Jimbo generators. For example for the $S L(2, \mathrm{C})$ group we can write the matrix elements of $\Omega_{ \pm}$as [30]

$$
\Omega_{+}=\left(\begin{array}{cc}
q^{-H / 2} & q^{-1 / 2} \lambda X_{+} \\
0 & q^{H / 2}
\end{array}\right), \Omega_{-}=\left(\begin{array}{cc}
q^{H / 2} & 0 \\
-q^{1 / 2} \lambda X_{-} & q^{-H / 2}
\end{array}\right) .
$$

Using the $R_{+}$matrix above it can be shown by direct computations that the generators $H, X_{ \pm}$satisfy the Jimbo-Drinfeld relations [28, 45]

$$
\left[H, X_{ \pm}\right]= \pm 2 X_{ \pm}, \quad\left[X_{+}, X_{-}\right]=\frac{q^{H}-q^{-H}}{q-q^{-1}}
$$

defining the universal enveloping algebra $\mathcal{U}_{q}(\operatorname{sl}(2, \mathrm{C}))$. Similar relations also exist for higher rank groups [30] and can be thought of as connecting the Cartan-Weyl and Chevalley bases.

It is also convenient to combine $\Omega_{ \pm}$into a single matrix [59]

$$
\Omega=\Omega_{+} \Omega_{-}^{-1} .
$$

In terms of these generators all the relations (7.3) and (7.4) collapse to

$$
\begin{aligned}
\Omega^{1} R_{-}^{-1} \Omega^{2} R_{-} & =R_{+}^{-1} \Omega^{2} R_{+} \Omega^{1} \\
R_{-} g^{1} \Omega^{2} & =\Omega^{2} R_{+} g^{1}
\end{aligned}
$$

These forms of the commutation relations are especially useful when we deal with the commutation relations only, but the coproduct of $\Omega$ cannot in general be given in an explicit form.

The commutation relations (7.2)(7.11) are exactly those satisfied by the local field and the monodromy of the left (or right) chiral component of the affine current $[2,4,31]$. 
Following [5] we also introduce an equivalent description of the quantum algebra using operators generating right translations. First let

$$
\Sigma=g^{-1} \Omega g
$$

and then introduce a triangular decomposition of $\Sigma$ into $\Sigma_{ \pm}$

$$
\Sigma=\Sigma_{+} \Sigma_{-}^{-1}
$$

similar to the decomposition of $\Omega$ into $\Omega_{ \pm}$. One can check that the matrix elements of $\Omega$ and $\Sigma$ commute. To make the picture more symmetric also introduce a new matrix $h$ by

$$
h=\Sigma_{ \pm}^{-1} g^{-1} \Omega_{ \pm}
$$

Now we can use either pair $(g, \Omega)$ or $(h, \Sigma)$ to describe the algebraT* $G_{q}$.

The defining relations satisfied by $h$ and $\Sigma$ are [5]

$$
\begin{aligned}
R_{ \pm} h^{1} h^{2} & =h^{2} h^{1} R_{ \pm} \\
\Sigma_{+}^{1} \Sigma_{+}^{2} R_{ \pm} & =R_{ \pm} \Sigma_{+}^{2} \Sigma_{+}^{1} \\
\Sigma_{-}^{1} \Sigma_{-}^{2} R_{ \pm} & =R_{ \pm} \Sigma_{-}^{2} \Sigma_{-}^{1} \\
\Sigma_{-}^{1} \Sigma_{+}^{2} R_{+} & =R_{+} \Sigma_{-}^{2} \Sigma_{-}^{1} \\
\Sigma_{+}^{1} \Sigma_{-}^{2} R_{-} & =R_{-} \Sigma_{-}^{2} \Sigma_{+}^{1} \\
h^{1} \Sigma_{+}^{2} & =\Sigma_{+}^{2} R_{-} h^{1} \\
h^{1} \Sigma_{-}^{2} & =\Sigma_{-}^{2} R_{+} h^{1} .
\end{aligned}
$$

One can check directly the consistency of (7.14) with the original relations. 


\subsection{Real Form for the $q$-Deformed Symmetric Top}

For a large number of applications the variable $q$ is a phase. In this case the $R_{ \pm}$matrices satisfy

$$
R_{+}^{\dagger}=R_{\sim} .
$$

If we require a reality structure for $g$ compatible with the Hopf algebra structure i.e.

$$
\triangle \circ *=(* \otimes *) \circ \Delta
$$

and use (7.15) we obtain a non-compact quantum group. For example if $G=$ $S L(N, \mathrm{C})$ we obtain $\operatorname{Fun}_{q}(S L(N, \mathrm{R}))$.

However sometimes in the same application we are interested in the compact form of the group. This apparent contradiction can be resolved [5] by dropping the above requirement for a Hopf $*$-structure. Instead one defines an anti-involution on the larger algebra $\mathrm{T}^{*} G_{q}$

$$
\begin{aligned}
\Omega_{ \pm}^{\dagger} & =\Omega_{\mp} \\
g^{\dagger} & =h .
\end{aligned}
$$

It is straightforward [5] to check the compatibility of this anti-involution with the quantum algebra $(7.2)(7.3)(7.4)(7.14)$. Note that (7.16) does not define a Hopf *-structure on $U_{q}(\mathfrak{g})$, and (7.17) does not close on $\operatorname{Fun}_{q}(G)$ since the definition of $h$ includes generators of $U_{q}(\mathfrak{g})$. In the classical limit (7.17) reduces to $g^{\dagger}=g^{-1}$ and (7.16) becomes compatible with the coproduct. This is due to the fact that the coproduct is cocommutative at $q=1$. 


\subsection{Real Form for the $q$-Deformed Hyperboloid}

This section contains the main result of this chapter, an anti-involution on the deformed cotangent bundle when $q$ is a phase. Like the anti-involution of the previous section, it does not originate from a Hopf $*$-structure on one of the Hopf subalgebras. The defining relations of the anti-involution are

$$
\begin{aligned}
g^{\dagger} & =g \\
\Omega_{ \pm}^{\dagger} & =\Sigma_{\mp}^{-1} .
\end{aligned}
$$

Alternatively the second relation can be written as

$$
\Omega^{\dagger}=\Sigma=g^{-1} \Omega g .
$$

It is quite obvious that (7.18) is not compatible with the coproduct, i.e. $g$ should not be considered a "group element". I will not give a complete proof of the consistency of the anti-involution with the algebra relations $(7.2)(7.3)(7.4)$. Instead I will just give a sample computation leaving the rest for the interested reader.

Applying the involution on the $R_{+}$relation (7.2) and using (7.15) we have

$$
\left(g^{2}\right)^{\dagger}\left(g^{1}\right)^{\dagger} R_{-}=R_{-}\left(g^{1}\right)^{\dagger}\left(g^{2}\right)^{\dagger} .
$$

Moving the $R_{-}$matrices to the other side and using (7.1) we obtain

$$
R_{+}\left(g^{1}\right)^{\dagger}\left(g^{2}\right)^{\dagger}=\left(g^{2}\right)^{\dagger}\left(g^{1}\right)^{\dagger} R_{+},
$$

thus it is consistent with the algebra relations (7.2) to impose $g^{\dagger}=g$. 
As another example, take the hermitian conjugate of the following relation

$$
R_{+} \Omega_{+}^{1} \Omega_{+}^{2}=\Omega_{+}^{2} \Omega_{+}^{1} R_{+} .
$$

Using (7.19) we obtain

$$
\left(\Sigma_{-}^{2}\right)^{-1}\left(\Sigma_{-}^{1}\right)^{-1} R_{-}=R_{-}\left(\Sigma_{-}^{1}\right)^{-1}\left(\Sigma_{-}^{2}\right)^{-1}
$$

which can be rewritten after multiplication by some inverse matrices as

$$
R_{-} \Sigma_{-}^{2} \Sigma_{-}^{1}=\Sigma_{-}^{1} \Sigma_{-}^{2} R_{-} .
$$

This is just one of the equations in (7.14).

Similarly applying the above involution on the first relation in (7:4) we obtain

$$
\begin{gathered}
g^{2}\left(\Sigma_{-}^{1}\right)^{-1} R_{-}=\left(\Sigma_{-}^{1}\right)^{-1} g^{2} \\
\Sigma_{-}^{1} g^{2}=g^{2} R_{-}^{-1} \Sigma_{-}^{1} .
\end{gathered}
$$

This is equivalent using (7.1) and (7.5) to

$$
\Sigma_{-}^{2} g^{1}=g^{1} R_{+} \Sigma_{-}^{2},
$$

and after eliminating $g$ using (7.13) we get

$$
\Sigma_{-}^{2} \Omega_{-}^{1}\left(h^{1}\right)^{-1}\left(\Sigma_{-}^{1}\right)^{-1}=\Omega_{-}^{1}\left(h^{1}\right)^{-1}\left(\Sigma_{-}^{1}\right)^{-1} R_{+} \Sigma_{-}^{2} .
$$

Furthermore using (7.14) to commute the $\Sigma$ matrices we have

$$
\Sigma_{-}^{2} \Omega_{-}^{1}\left(h^{1}\right)^{-1}=\Omega_{-}^{1}\left(h^{1}\right)^{-1} \Sigma_{-}^{2} R_{+}
$$


and since $\Omega$ and $\Sigma$ commute with each other we finally obtain

$$
h^{1} \Sigma_{-}^{2}=\Sigma_{-}^{2} R_{+} h^{1}
$$

which is again one of the relations in (7.14). All the other relations can be checked in a similar fashion.

Finally I will explain the terminology used in the title of this section. Consider first for simplicity the $S L(2, \mathrm{C})$ case. In the undeformed case a $2 \times 2$ hermitian matrix of unit determinant defines the unit mass hyperboloid in Minkowski space. For simplicity I will only consider one connected component of the manifold, for example the future mass hyperboloid. For a general group $G$ this can be achieved by restricting to positive definite matrices. In the deformed case we consider Hermitian matrices $g$ of unit quantum determinant.

\subsection{Quantum Mechanics on the $q$-Deformed Hyperboloid}

In [3] Alekseev and Faddeev showed that the $\mathrm{T}^{*} G_{q}$ quantum algebra is a $q$ deformation of the algebra of functions on the cotangent bundle of the Lie group $G$. In [5] they considered the following simple Lagrangian written in first order formalism

$$
\mathcal{L}=\operatorname{Tr}\left(\omega \dot{g} g^{-1}-\frac{1}{2} \omega^{2}\right)
$$

Here $G$ is considered without specifying its real form. The Lagrangian has a chiral symmetry $G \times G$

$$
g \rightarrow u g v^{-1}, \quad \omega \rightarrow u \omega v^{-1}, \quad u, v \in G
$$


The second order form of the Lagrangian has the form of a non-linear sigma model in $(0,1)$ dimensions

$$
\mathcal{L}=\frac{1}{2} \operatorname{Tr}\left(\dot{g} g^{-1} \dot{g} g^{-1}\right)
$$

The equations of motion

$$
\dot{g}=\omega g, \quad \dot{\omega}=0
$$

can be integrated to give the time evolution

$$
\begin{aligned}
& \omega(t)=\omega(0) \\
& g(t)=\exp (\omega t) g(0) .
\end{aligned}
$$

The real form corresponding to the compact group discussed in [5] is

$$
g^{\dagger}=g^{-1}, \quad \omega^{\dagger}=-\omega
$$

For $G=S L(2, \mathrm{C}), g$ becomes unitary and the Lagrangian (7.22) describes the classical dynamics of the symmetric top. Equivalently, it describes the motion on a constant curvature $S^{3}$. This can be seen using the chiral symmetry (7.4) of the Lagrangian, which under the conditions (7.24) is restricted to the $S U(2) \times$ $S U(2) \sim S O(4)$ subgroup, or by direct computation of the metric in the kinetic term of (7.23).

Instead, we consider the following reality structure

$$
g^{\dagger}=g, \quad \omega^{\dagger}=g^{-1} \omega g
$$

which, following the discussion at the end of the previous section, defines the phase space of a particle moving on the mass-hyperboloid. The reality structure (7.25) 
requires $u^{\dagger}=v^{-1}$ thus restricting the chiral symmetry of the Lagrangian to one independent $S L(2, \mathrm{C})$ subgroup which is simply the Lorentz group that leaves the mass hyperboloid invariant. The metric on the hyperboloid is just the induced metric from Minkowski space, and again this can be obtained by direct computation or using the above invariance under the Lorentz group.

One can check that the equations of motion preserve both reality structures (7.24) and (7.25). What we learn from this simple example is that one can find rather different physical systems that will have the same Poisson brackets and thus quantum algebras if their respective Lagrangians have the same form, differing only through their reality structures.

In [5] a $q$-deformation of the above system was introduced. The model has a discrete time dynamics, with the time labelled by an integer $n$. The following evolution equations

$$
\begin{aligned}
& \Omega(n)=\Omega(0) \\
& g(n)=\Omega^{n} g(0)
\end{aligned}
$$

were shown in [5] to preserve the quantum algebra $(7.2)(7.3)(7.4)$ and in addition, the reality structure discussed in Section 7.2 .

I will now show that they also preserve the reality structure introduced in Section 7.3. Assuming that for $n=0$ the reality structure is given by (7.18) and (7.20)

$$
g^{\dagger}(0)=g(0), \quad \Omega^{\dagger}(0)=g^{-1}(0) \Omega(0) g(0)
$$


for arbitrary $n$ we have

$$
\left.g^{\dagger}(n)=g^{\dagger}(0)\left(\Omega^{\dagger}(0)\right)^{n}=g(0)\left(g^{-1}(0) \Omega(0)\right) g(0)\right)^{n}=\Omega^{n}(0) g(0)=g(n)
$$

Similarly we have for $\Omega(n)$

$$
\Omega^{\dagger}(n)=\Omega^{\dagger}(0)=g^{-1}(0) \Omega(0) g(0)=g^{-1}(n) \Omega(0) g(n)=g^{-1}(n) \Omega(n) g(n)
$$

Thus the equations of motion (7.26) and the reality structure of the previous Section define the $q$-deformation of the dynamics of a particle on the unit mass hyperboloid.

\subsection{Lattice regularized WZNW-model}

I conclude by briefly applying the reality structure to the lattice regularized WZNW-model and checking its compatibility with periodic boundary conditions. Using the notation in [2] let the lattice have $N$ points, and denote the local fields by $g_{i}, i=1 \ldots N$. For periodic boundary conditions we identify $i$ and $i+N$. Let $M_{L}$ and $M_{R}$ be the monodromies of the left and right affine currents. The algebra satisfied by $\left(g, M_{L}, M_{R}\right)$ is exactly the algebra of $\mathrm{T}^{*} G_{q}$ for the generators $(g, \Omega, \Sigma)$. Here I used the remark of the previous Section that the compact and non-compact WZNW-models have the same algebra since their respective Lagrangians coincide. The monodromies can be used to relate the fields $g_{0}$ and $g_{N}$

$$
g_{N}=M_{L} g_{0} M_{R}^{-1}
$$


If we require $g_{0}^{\dagger}=g_{0}, M_{L}^{\dagger}=M_{R}$, which is just the reality structure of Section 7.3 , we have

$$
g_{N}^{\dagger}=\left(M_{R}^{-1}\right)^{\dagger} g_{0} M_{L}^{\dagger}=M_{L}^{-1} g_{0} M_{R}=g_{-N}=g_{N}
$$

In the last step I used the lattice periodicity. Thus we see that the reality structure is compatible with periodic boundary conditions. 


\section{Appendix A}

\section{Chiral Spinor Representations of $S O(d, d \mid \mathbf{Z})$}

In the first part of this appendix it will be shown that the Weyl spinor representations of $S O(d, d \mid \mathrm{Z})$ are integral, i.e. have matrix elements which are integers. In the final part, it is proven that for $d=3$ the Weyl spinor representation is in fact isomorphic to $S L(4, \mathbf{Z})$.

The gamma matrices obeying (2.47), where the metric has the form (2.45), are already, up to normalization, the standard creation and annihilation operators used to generate the Fock space for Dirac spinors in the Weyl basis. These are defined as

$$
a_{i}^{\dagger}=\gamma_{i} / \sqrt{2}, \quad a_{i}=\gamma_{d+i} / \sqrt{2}
$$

lblletter/ and satisfy the canonical anti-commutation relations

$$
\left\{a_{i}, a_{j}^{\dagger}\right\}=\delta_{i j}, \quad\left\{a_{i}, a_{j}\right\}=\left\{a_{i}^{\dagger}, a_{j}^{\dagger}\right\}=0, \quad i, j=1, \ldots, d .
$$

As usual, the Dirac spinor and vector representations are related through formula (2.46) in the main text

$$
\mathcal{S}^{-1} \gamma_{s} \mathcal{S}=\Lambda_{s}^{p} \gamma_{p}
$$

To prove that the Weyl spinor representations are integral I will use a theorem presented in [68] where it was shown that the whole group $S O(d, d \mid \mathbf{Z})$ is generated 
by a special subset of group elements. An explicit construction of the Weyl spinor representation matrices corresponding to the group elements in that subset is given and shown to be integral. The subset contains three types of elements. The first type are generators of the form

$$
\left(\begin{array}{cc}
I_{d} & n \\
0 & I_{d}
\end{array}\right), n^{T}=-n .
$$

The second type of generators forming a $S L(d, \mathbf{Z}) \times \mathbf{Z}_{2}$ subgroup have the form

$$
\left(\begin{array}{cc}
R & 0 \\
0 & R^{T^{-1}}
\end{array}\right), \quad \operatorname{det} R= \pm 1 .
$$

These are the T-duality generators corresponding to a change of basis of the of the compactification lattice.

The final generator is given by

$$
\left(\begin{array}{llllll}
0 & & & 1 & & \\
& 0 & & & 1 & \\
& & I_{d-2} & & & 0_{d-2} \\
& & & 0 & & \\
& & & & 0 & \\
& 1 & & & 0 & \\
& & 0_{d-2} & & & I_{d-2}
\end{array}\right) .
$$

It corresponds to T-duality along the $x^{1}$ and $x^{2}$ coordinates. The full duality group is in fact $O(d, d \mid \mathbf{Z})$ but here I will only consider its restriction to $S O(d, d \mid \mathbf{Z})$ which is the subgroup that does not exchange Type IIA and IIB. The full Tduality group is then obtained by adding to the above list one more generator corresponding to T-duality in a single direction.

Using (2.46) one can check that the Dirac spinor representation corresponding 
to the first type of generator (A.1) is

$$
\exp \left(\frac{1}{2} n_{i j} a_{i} a_{j}\right)
$$

This has a finite expansion and is manifestly integer valued in the standard Fock space basis obtained by acting with the creation operators on a vacuum state.

One can prove that the full $S L(d, \mathbf{Z})$ group is generated by its $S L(2, \mathbf{Z})_{i j}$ subgroups acting on the $x^{i}$ and $x^{j}$ coordinates. One can use this to find the spinor representation matrices corresponding to generators of the second type (A.2). Since each $S L(2, \mathbf{Z})_{i j}$ is generated by its $T_{i j}$ and $S_{i j}$ transformations, which in the $(i j)$ subspace where $i<j$ have the form

$$
\left(\begin{array}{ll}
1 & 1 \\
0 & 1
\end{array}\right), \quad\left(\begin{array}{cc}
0 & -1 \\
1 & 0
\end{array}\right),
$$

it is enough to find the spinor matrices for these generators. The spinor representation of $T_{i j}$ is given by

$$
\exp \left(a_{i} a_{j}^{\dagger}\right)
$$

The exponential (A.5) has a finite expansion and its matrix elements are integer valued. Similarly the spinor representation of $S_{i j}$ is given by

$$
\exp \left(\frac{\pi}{2}\left(a_{j}^{\dagger} a_{i}-a_{i}^{\dagger} a_{j}\right)\right)
$$

Let us define $A=a_{j}^{\dagger} a_{i}-a_{i}^{\dagger} a_{j}$ for fixed values of $i$ and $j$. In terms of number operators $N_{i}=a_{i}^{\dagger} a_{i}$ we have $A^{2}=-N_{i}-N_{j}+2 N_{i} N_{j}$. Since $N_{i}$ can be either zero or one, $A^{2}$ is zero or minus one. The Fock space can be split into a direct sum of two subspaces, defined by the eigenvalues of $A^{2}$. On the subspace defined by 
$A^{2}=0$, one also has $A=0$ and thus the spinor representation (A.6) reduces to the identity. On the subspace defined by $A^{2}=-1$, the exponential can be written as $\cos (\pi / 2)+A \sin (\pi / 2)=A$. On both subspaces, the representation matrix of the transformation is integer valued. A formula for the spinor representation of the $S_{i j}$ generators which is valid on both subspaces is given by, $1+A+A^{2}$. The second type of generator (A.2) also contains elements with $\operatorname{det} R=-1$. A spinor transformation corresponding to such a generator is given by

$$
1-2 a_{1}^{\dagger} a_{1}
$$

Finally, the generator (A.3) has the spinor representation

$$
\exp \left(\frac{\pi}{2}\left(a_{1}-a_{1}^{\dagger}\right)\left(a_{2}-a_{2}^{\dagger}\right)\right)
$$

It has a finite expansion given by $\left(a_{1}-a_{1}^{\dagger}\right)\left(a_{2}-a_{2}^{\dagger}\right)$, which can be obtained using $\left(\left(a_{1}-a_{1}^{\dagger}\right)\left(a_{2}-a_{2}^{\dagger}\right)\right)^{2}=-1$, and in this form it is manifestly integral.

Since the Fock space basis used splits into two subsets of definite chirality, it follows that the Weyl spinor representations of $S O(d, d \mid \mathbf{Z})$ are also integral.

In the remainder of the appendix it is shown that the Weyl spinor representation of $S O(3,3 \mid \mathbf{Z})$ is isomorphic to $S L(4, \mathbf{Z})$. First note that for the Lie algebra corresponding to the continuous Lie groups we have the equivalence $s o(3,3 \mid \mathbf{R}) \cong s l(4, \mathbf{R})$. The spinor representation of the first group is isomorphic to the fundamental of the second. Since in the first part of the appendix it was shown that the spinor representations are integral it is reasonable to expect that they form a subgroup of $S L(4, \mathbf{Z})$. In fact I will show that they are isomorphic 
to the whole $S L(4, \mathrm{Z})$ group.

One can represent the Weyl spinor state $n|0\rangle+\frac{1}{2} M^{i j} a_{i}^{\dagger} a_{j}^{\dagger}|0\rangle$ as the column

$$
\left(\begin{array}{c}
n \\
M^{23} \\
M^{31} \\
M^{12}
\end{array}\right)
$$

Using operators of the form (A.5) and (A.6) one generates an $S L(3, \mathbf{Z})$ subgroup of the form

$$
\left(\begin{array}{ll}
1 & 0 \\
0 & R
\end{array}\right)
$$

where $R$ is the same matrix appearing in (A.2). I will now show that the Weyl spinor representation also contains $S L(2, \mathrm{Z})_{1 i}$ subgroups which act on the first and the $i+1$ entries of the column spinor (A.9). These subgroups together with (A.10) generate the entire $S L(4, \mathbf{Z})$ group. The T-duality generator (A.8), denoted below $T_{12}$, has the Weyl spinor representation

$$
T_{12}=\left(\begin{array}{cccc}
0 & 0 & 0 & -1 \\
0 & 0 & -1 & 0 \\
0 & 1 & 0 & 0 \\
1 & 0 & 0 & 0
\end{array}\right)
$$

Let us also consider a transformation $G$ given by

$$
G=\left(\begin{array}{cccc}
1 & 0 & 0 & 0 \\
0 & 1 & 0 & 0 \\
0 & 0 & a & b \\
0 & 0 & c & d
\end{array}\right), \quad a d-b c=1
$$

which is an element of an $S L(2, \mathrm{Z})$ subgroup of elements of the form (A.10). By 
conjugating $G$ with the $T_{12}$ generator

$$
T_{12}^{-1} G T_{12}=\left(\begin{array}{cccc}
d & c & 0 & 0 \\
b & a & 0 & 0 \\
0 & 0 & 1 & 0 \\
0 & 0 & 0 & 1
\end{array}\right)
$$

one finds an $S L(2, \mathbf{Z})_{12}$ transformation acting on the first and second entries. All the other $S L(2, Z)_{1 i}$ subgroups can be obtained by conjugating (A.11) with elements of the form (A.10). Thus we have found Weyl spinor representations generating the entire $S L(4, \mathrm{Z})$ group. In fact the representation is isomorphic to $S L(4, \mathbf{Z})$ since all the spinor representation matrices (A.4), (A.5), (A.6), (A.7) and (A.8) are integral and have unit determinant. 


\section{Appendix B}

\section{Duality Invariants at Small Compactification}

\section{Volume}

This Appendix contains some mathematical results regarding the spinor representation of the T-duality group and duality invariant quantities in the small volume limit. The group $S O(d, d)$ is the group of $2 d$-dimensional matrices $\Lambda$ satisfying $\Lambda J \Lambda^{T}=J$ where $J$ is a matrix with the block form

$$
J=\left(\begin{array}{ll}
0 & 1 \\
1 & 0
\end{array}\right)
$$

It will be useful to know how to calculate the Weyl spinor representation matrix of an $S O(3,3)$ group element $\Lambda$ with the block form

$$
\Lambda=\left(\begin{array}{ll}
\mathcal{A} & \mathcal{B} \\
\mathcal{C} & \mathcal{D}
\end{array}\right)
$$

First note that if $\mathcal{A}$ is invertible $\Lambda$ has a block Gauss decomposition

$$
\Lambda=\left(\begin{array}{cc}
1 & 0 \\
\mathcal{C A}^{-1} & 1
\end{array}\right)\left(\begin{array}{cc}
\mathcal{A} & 0 \\
0 & \mathcal{A}^{-T}
\end{array}\right)\left(\begin{array}{cc}
1 & \mathcal{A}^{-1} \mathcal{B} \\
0 & 1
\end{array}\right),
$$

where one can show using the group relations that $\mathcal{C} \mathcal{A}^{-1}$ and $\mathcal{A}^{-1} \mathcal{B}$ are antisymmetric. This decomposition is in fact true for generic $S O(d, d)$ matrices. For $d=3$ one can give the explicit spinor representation matrices for each factor in (B) thus 
obtaining the spinor representation of a generic $S O(3,3)$ matrix $\Lambda$

$$
S=\left(\begin{array}{cc}
1 & 0 \\
*\left(\mathcal{C A}^{-1}\right) & 1
\end{array}\right)\left(\begin{array}{cc}
\operatorname{det} \mathcal{A}^{1 / 2} & 0 \\
0 & \operatorname{det} \mathcal{A}^{-1 / 2} \mathcal{A}
\end{array}\right)\left(\begin{array}{cc}
1 & *\left(\mathcal{A}^{-1} \mathcal{B}\right)^{T} \\
0 & 1
\end{array}\right)
$$

The star denotes the duality operator. When acting on antisymmetric 3dimensional square matrices it gives the dual column matrix.

Invariants can be constructed using two column matrices transforming in the vector representation of $S O(d, d \mid \mathbf{Z})$ and the symmetric $S O(d, d \mid \mathbf{Z})$ matrix $\mathcal{M}$

$$
\left(r^{T}, s^{T}\right) \mathcal{M}\left(\begin{array}{l}
u \\
v
\end{array}\right)
$$

In the limit when $G$ goes to zero, using the block Gauss decomposition of $\mathcal{M}$

$$
\mathcal{M}=\left(\begin{array}{ll}
1 & 0 \\
B & 1
\end{array}\right)\left(\begin{array}{cc}
G^{-1} & 0 \\
0 & G
\end{array}\right)\left(\begin{array}{cc}
1 & -B \\
0 & 1
\end{array}\right)
$$

and after identifying $B$ with $\Theta$ one obtains the following invariant ${ }^{1}$

$$
(r-\Theta s)^{T} G_{r}^{-1}(u-\Theta v)
$$

Using the transformation of $G$ under the duality group (1.12) one can write the transformation of $u-\Theta v$

$$
(\bar{u}-\bar{\Theta} \bar{v})=(\mathcal{C} \Theta+\mathcal{D})^{-T}(u-\Theta v)
$$

The spinor representation matrix of $\mathcal{M}$ can be calculated using (B.2)

$$
S(\mathcal{M})=\left(\begin{array}{ll}
1 & 0 \\
b & 1
\end{array}\right)\left(\begin{array}{cc}
\operatorname{det} G^{-1 / 2} & 0 \\
0 & \operatorname{det} G^{1 / 2} G^{-1}
\end{array}\right)\left(\begin{array}{cc}
1 & b^{T} \\
0 & 1
\end{array}\right) .
$$

\footnotetext{
${ }^{1}$ To obtain a finite result, one should insert appropriate factors of $\alpha^{\prime}$ in (B.3) and also take $\alpha^{\prime}$ to zero as discussed in the Introduction.
} 
Then one can also form the invariants $\eta^{T} S(\mathcal{M}) \chi$ using two chiral spinors

$$
\chi=\left(\begin{array}{c}
\chi_{0} \\
\chi_{1} \\
\chi_{2} \\
\chi_{3}
\end{array}\right), \quad \eta=\left(\begin{array}{c}
\eta_{0} \\
\eta_{1} \\
\eta_{2} \\
\eta_{3}
\end{array}\right) .
$$

In the limit of vanishing $G$, the invariant becomes

$$
\left(\eta_{0}+\frac{1}{2} \varepsilon^{i j k} \Theta_{i j} \eta_{k}\right) \sqrt{\operatorname{det} G^{-1}}\left(\chi_{0}+\frac{1}{2} \varepsilon^{i j k} \Theta_{i j} \chi_{k}\right)
$$

From (B.5) one obtains the following transformation law

$$
\bar{\chi}_{0}+\frac{1}{2} \varepsilon^{i j k} \bar{\Theta}_{i j} \bar{\chi}_{k}=|\operatorname{det}(\mathcal{C} \Theta+\mathcal{D})|^{-1 / 2}\left(\chi_{0}+\frac{1}{2} \varepsilon^{i j k} \Theta_{i j} \chi_{k}\right) .
$$

One can also check the relations (B.4) and (B.6) directly using the transformations (1.11) of $\Theta$.

Given two chiral spinors $\chi$ and $\eta$ first write them as Dirac spinors

$$
\chi_{D}=\left(\begin{array}{c}
\chi \\
0
\end{array}\right), \quad \eta_{D}=\left(\begin{array}{l}
\eta \\
0
\end{array}\right) .
$$

Then using the same definition for $a^{i}$ and $a_{i}^{\dagger}$ as in Appendix A one can form the $S O(3,3 \mid \mathbf{Z})$ vector

$$
\left(\begin{array}{c}
u_{i} \\
v^{i}
\end{array}\right)=\bar{\eta}_{D}\left(\begin{array}{c}
a_{i}^{\dagger} \\
a^{i}
\end{array}\right) \chi_{D} .
$$

where $\vec{\eta}_{D}=\eta^{\dagger} T$. Here $T$ is a matrix acting on Dirac spinors and plays the same role as $\gamma_{0}$ when one forms barred spinors in Minkowski space. It is given by

$$
T=\left(a_{1}^{\dagger}+a^{1}\right)\left(a_{2}^{\dagger}+a^{2}\right)\left(a_{3}^{\dagger}+a^{3}\right) .
$$

Writing out all the spinor components in (B.7) one has

$$
\left(\begin{array}{c}
u_{i} \\
v^{i}
\end{array}\right)=\left(\begin{array}{c}
\eta_{i} \chi_{0}-\eta_{0} \chi_{i} \\
\varepsilon^{i j k} \eta_{j} \chi_{k}
\end{array}\right) .
$$


Then $u-\Theta v$ transforms as in (B.4) under the duality group. Such an expression, involving two chiral spinors and $\Theta$, is used in the main text. 


\section{Appendix C}

\section{Dual Quantum Groups and Universal}

\section{Enveloping Algebras}

Here we list some relations defining the quantum group $F u n_{q}\left(S U(2)^{*}\right)$ and discuss its relation to $\mathcal{U}_{q}(s u(2))[28,30,88]$. We only discuss the algebra and ignore all other issues. The quantum group $F u n_{q}\left(S U(2)^{*}\right)$ is a factorizable quasitriangular Hopf algebra. As an algebra it is generated by triangular matrices $L^{ \pm}$ satisfying quantum commutation relations

$$
\begin{aligned}
& R_{ \pm} L_{1}^{ \pm} L_{2}^{ \pm}=L_{2}^{ \pm} L_{1}^{ \pm} R_{ \pm} \\
& R_{+} L_{1}^{+} L_{2}^{-}=L_{2}^{-} L_{1}^{+} R_{+} \\
& R_{-} L_{1}^{-} L_{2}^{+}=L_{2}^{+} L_{1}^{-} R_{-}
\end{aligned}
$$

where

$$
R_{+}=q^{-1 / 2}\left(\begin{array}{cccc}
q & 0 & 0 & 0 \\
0 & 1 & \lambda & 0 \\
0 & 0 & 1 & 0 \\
0 & 0 & 0 & q
\end{array}\right), R_{-}=q^{1 / 2}\left(\begin{array}{cccc}
q^{-1} & 0 & 0 & 0 \\
0 & 1 & 0 & 0 \\
0 & -\lambda & 1 & 0 \\
0 & 0 & 0 & q^{-1}
\end{array}\right)
$$

The universal enveloping algebra $\mathcal{U}_{q}(s u(2))$ is a quasi-triangular Hopf algebra. It has generators $H, X_{ \pm}$which satisfy the Jimbo-Drinfeld relations

$$
\left[H, X_{ \pm}\right]= \pm 2 X_{ \pm},\left[X_{+}, X_{-}\right]=\frac{q^{H}-q^{-H}}{q-q^{-1}}
$$


In [30] it was shown that these two Hopf algebras are isomorphic. The isomorphism is given by

$$
L^{+}=\left(\begin{array}{cc}
q^{-H / 2} & q^{-1 / 2} \lambda X_{+} \\
0 & q^{H / 2}
\end{array}\right), \quad L^{-}=\left(\begin{array}{cc}
q^{H / 2} & 0 \\
-q^{1 / 2} \lambda X_{-} & q^{-H / 2}
\end{array}\right) .
$$

As in the classical case we can define the matrix $L=\left(L^{-}\right)^{-1} L^{+}$. It satisfies the following equation:

$$
R_{+}^{-1} L_{1} R_{+} L_{2}=L_{2} R_{-}^{-1} L_{1} R_{-}
$$

as can be checked using (6.22).

In the classical limit we define $r_{ \pm}$matrices by $R_{ \pm}=1+\hbar r_{ \pm}+\mathcal{O}\left(\hbar^{2}\right)$. Then

$$
\left(1-\hbar r_{+}\right) L_{1}\left(1+\hbar r_{+}\right) L_{2}=L_{2}\left(1-\hbar r_{-}\right) L_{1}\left(1+\hbar r_{-}\right)+\mathcal{O}\left(\hbar^{2}\right)
$$

and we obtain the following Poisson structure

$$
\left\{L_{1}, L_{2}\right\} \equiv \lim _{\hbar \rightarrow 0} \frac{\left[L_{1}, L_{2}\right]}{-\hbar}=+L_{1} r_{+} L_{2}+L_{2} r_{-} L_{1}-r_{+} L_{1} L_{2}-L_{1} L_{2} r_{-}
$$

This is just the original Poisson bracket (6.6) which was the starting point for the path integral quantization. 


\section{Bibliography}

[1] A, Alekseev, L. Faddeev, S. Shatashvili, Quantization of Symplectic Orbits of Compact Lie Groups by Means of the Functional Integral, JGP. Vol. 5, nr. 3 (1989) $391-406$

[2] A. Yu. Alekseev, L.D. Faddeev, M. A. Semenov-Tian-Shansky, A. Volkov The Unraveling of the Quantum Group Structure in the WZNW theory, Preprint CERN-TH-5981/91, January1991

[3] A. Yu. Alekseev, L.D. Faddeev $\left(\mathrm{T}^{*} G\right)_{t}: A$ Toy Model for Conformal Field Theory, Commun. Math. Phys. 141 (1991) 413-422

[4] A. Yu. Alekseev, L.D. Faddeev, M. A. Semenov-Tian-Shansky Hidden Quantum Groups Inside Kac-Moody Algebra, Commun. Math. Phys. 149 (1992) $335-345$

[5] A. Yu. Alekseev, L.D. Faddeev An Involution and Dynamics for the $q$ Deformed Quantum Top, Preprint hep-th/9406196, June 1994

[6] F. Ardalan, H. Arfaei, M. M. Sheikh-Jabbari, Noncommutative Geometry from Strings and Branes, hep-th/9810072

[7] F. Ardalan, H. Arfaei, M. M. Sheikh-Jabbari, Mixed Branes and M(atrix) Theory on Noncommutative Torus, hep-th/9803067 
[8] M. Baake, P. Reinicke, V. Rittenberg, Fierz Identities for Real Clifford Algebras and the Number of Supercharges, J. Math. Phys. 26 (1985) 1070

[9] T. Banks, W. Fishler, S.H. Shenker, L Susskind, M Theory As A Matrix Model: A Conjecture, Phys. Rev. D55 (1997) 5112-5128; hep-th/9610043

[10] D. Berenstein, R. Corrado, J. Distler, On the Moduli Spaces of M(atrix)Theory Compactifications, Nucl. Phys. B503 (1997) 239-255; hep-th/9704087

[11] E. Bergshoeff, C. M. Hull, T. Ortin, Duality in the Type-II Superstring Effective Action, Nucl. Phys. B451 (1995) 547-578; hep-th/9504081

[12] D. Bigatti, Noncommutative Geometry for Outsiders, hep-th/9802129

[13] D. Bigatti, Non commutative geometry and super Yang-Mills theory, hepth/9804120

[14] D. Brace, B. Morariu, B. Zumino, Dualities of the Matrix Model from T-Duality of the Type II String, hep-th/9810099

[15] D. Brace, B. Morariu, A note on the BPS Spectrum of the Matrix Model, hep-th/9810185

[16] D. Brace, B. Morariu, B. Zumino, Dualities of the Matrix Model from T-Duality of the Type II String, hep-th/9810099

[17] R. Casalbuoni, Algebraic Treatment of Compactification on Noncommutative Tori, hep-th/9801170 
[18] C. S. Chu, P. M. Ho, B. Zumino, The Quantum 2-sphere as a Complex Quantum Manifold, Zf. Physik C70 (1996), 339; q-alg/9504003, April 1995

[19] C. S. Chu, P. M. Ho, B. Zumino, The Braided Quantum 2-Sphere, Mod. Phys. Lett. A11 (1996) 307-316; q-alg/9507013, July 1995

[20] M. Claudson, M. Halpern, Supersymmetric Ground State Wave Functions, Nucl. Phys. B250 (1985) 689

[21] Y.-K. E. Cheung, M. Krogh, Noncommutative Geometry from 0-branes in a Background B-field, hep-th/9803031

[22] A. Connes, M. Rieffel, Yang-Mills for noncommutative two-Tori, Operator Algebras and Mathematical Physics (Iowa City, Iowa, 1985) 237-266, Contemp. Math. Oper. Algebra. Math. Phys. 62, AMS 1987

[23] A. Connes, Noncommutative Geometry, Academic Press, 1994

[24] A. Connes, M. R. Douglas, A. Schwarz, Noncommutative Geometry and Matrix Theory: Compactification on Tori, JHEP02 (1998) 003; hep-th/9711162

[25] C. Destri, H. J. De Vega On The Connection Between The Principal Chiral Model and the Multiflavour Chiral Gross-Neveu Model, Phys. Lett. B 201 (1988) $245-250$

[26] M. R. Douglas, D. Kabat, P. Pouliot, S. H. Shenker, D-branes and Short Distances in String Theory, Nucl. Phys. B485 (1997) 85-127; hep-th/9608024 
[27] M. R. Douglas, C. Hull, D-branes and the Noncommutative Torus, JHEP02 (1998) 008; hep-th/9711165

[28] V. G. Drinfeld, Quantum Groups, ICM MSRI, Berkeley (1986) 798-820

[29] E. Eyras, B. Janssen, Y. Lozano, 5-branes, KK-monopoles and T-duality, Nucl. Phys. B531 (1998). 275-301; hep-th/9806169

[30] L.D. Faddeev, N. Yu. Reshetikhin, L. A. Takhtajan Quantization of Lie Groups and Lie Algebras, Alg. i Anal. 1 (1989) 178

[31] L.D. Faddeev, From Integrable Models to Conformal Field Theory via Quantum Groups, Integrable Systems, Quantum Groups, and Quantum Field Theory, L. A. Ibort, M. A. Rodriquez (eds.)

[32] R. Flume, On Quantum Mechanics with Extended Supersymmetry and Nonabelian Gauge Constraints, Ann. Phys. 164 (1985) 189

[33] O. J. Ganor, S. Ramgoolam, W. Taylor IV, Branes, Fluxes and Duality in M(atrix)-Theory, Nucl. Phys. 492, (1997) 191-204

[34] K. Gawędzki, Non-Compact WZW Conformal Field Theories, Preprint hepth/9110076, October 1991

[35] A. Giveon, M. Porrati and E. Rabinovici, Target Space Duality in String Theory, Phys. Rept. 244 (1994) 77-202; hep-th/9401139 
[36] M. B. Green, C. M. Hull, P. K. Townsend, D-brane Wess-Zumino actions, T-duality and the cosmological constant Phys. Lett. B382 (1996) 65-72; hepth/9604119

[37] P.-M. Ho, Y.-Y. Wu, Y.-S. Wu, Towards a Noncommutative Geometric Approach to Matrix Compactification, hep-th/9712201

[38] P.-M. Ho, Y.-S. Wu, Noncommutative Gauge Theories in Matrix Theory, hep-th/9801147

[39] P.-M. Ho, Twisted Bundle on Quantum Torus and BPS States in Matrix Theory, hep-th/9803166

[40] C. Hofman, E. Verlinde, G. Zwart, U-Duality Invariance of the Fourdimensional Born-Infeld Theory, hep-th/9808128

[41] C. Hofman, E. Verlinde, U-Duality of Born-Infeld on the Noncommutative Two-Torus, hep-th/9810116

[42] C. Hofman, E. Verline, Gauge Bundles and Born-Infeld on the Noncommutative Torus, hep-th/9810219

[43] C. M. Hull, Matrix Theory, U-Duality and Toroidal Compactifications of M-Theory, hep-th/9711179

[44] N. Ishibashi, H. Kawai, Y. Kitazawa, A. Tsuchiya, A Large N Reduced Model as Superstring, Nucl. Phys. B498 (1997) 467-491 
[45] M. Jimbo A q-Difference Analogue of $\mathrm{U}(\mathrm{g})$ of the Yang-Baxter Equation, Lett. Math. Phys. 10 (1985) 63-69

[46] T. Kawano, K. Okuyama, Matrix Theory on Noncommutative Torus, hepth/9803044

[47] A. A. Kirillov, Elements of the Theory of Representation, Berlin, Heidleberg, New York: Springer 1976

[48] A. Konechny, A. Schwarz BPS states on noncommutative tori and duality, hep-th/9811159

[49] G. Landi, F. Lizzi, R. J. Szabo, String Geometry and Noncommutative Torus, hep-th/9806099

[50] M. Li, Boundary States of D-Branes and D-Strings Nucl. Phys. B460 (1996) $351 ;$ hep-th/9510161

[51] J. H. Lu, A. Weinstein, Poisson-Lie Groups, Dressing transformations and Bruhat Decompositions, J. Diff. Geom. 31 (1990) 510

[52] B. Morariu, B. Zumino, Super Yang-Mills on the Noncommutative Torus, hep-th/9807198

[53] H. B. Nielsen, D. Rohrlich, A Path Integral to Quantize Spin, Nucl. Phys. B299 (1988) 471-483 
[54] N. A. Obers, B. Pioline, E. Rabinovici $M$-theory and U-duality on $T^{d}$ with gauge backgrounds, Nucl. Phys. B525 (1998) 163; hep-th/9712084

[55] N. A. Obers, B. Pioline, U-duality and M-theory, hep-th/9809039

[56] B. Pioline, E. Kiritsis, U-duality and D-brane Combinatorics, Phys. Lett. B418 (1998) 61-69; hep-th/9710078

[57] P. Podleś, Quantum Spheres, Lett. Math. Phys. 14 (1987) 193

[abs, src, ps, other] :

[58] J. Polchinski, Dirichlet-Branes and Ramond-Ramond Charges, Phys. Rev. Lett. 75 (1995) 4724-4727; hep-th/9510017

[59] N. Yu. Reshetikhin, M. A. Semenov-Tian-Shansky, Quantum R-matrices and Factorization Problems, JGP. Vol. 5, nr. 4 (1988) 534-550

[60] M. A. Rieffel, Induced Representations of $C^{*}$-algebras, Advances Math. 13 (1974) $176-257$

[61] M. A. Rieffel, $\mathbf{C}^{*}$-Algebras Associeted with Irrational Rotations, Pacific J. Math. 93 (1981) 415-429

[62] M. A. Rieffel, The Cancellation Theorem for Protective Modules over Irrational Rotations $\mathbf{C}^{*}$-Algebras, Proc. London Math. Soc. (3), 47 (1983) $285-302$ 
[63] M. A. Rieffel, Morita Equivalence for Operator Algebras, Operator Algebras and Applications, (R. V. Kadison, ed.) Proc. Symp. Pure Math. 38, Amer. Math. Soc., Providence, 1982

[64] M. A. Rieffel, Projective Modules over Higher-Dimensional Noncommutative Tori, Can. J. Math., XL No. 2 (1988) 257-338

[65] M. A. Rieffel, The Cancellation Theorem for Protective Modules over Irrational Rotations $\mathbf{C}^{*}$-Algebras, Proc. London Math. Soc. (3), 47 (1983) $285-302$

[66] M. A. Rieffel, Projective Modules over Higher-Dimensional Noncommutative Tori, Can. J. Math., XL No. 2 (1988) 257-338

[67] M. Rieffel, A. Schwarz, Morita equivalence of multidimensional noncommutative tori, math.QA/98033057

[68] A. Schwarz, Morita equivalence and duality, hep-th/9805034

[69] N. Seiberg, Why is the Matrix Model Correct?, Phys. Rev. Lett. 79 (1997) 3577-3580; hep-th/9710009

[70] M. A. Semenov-Tian-Shansky, Dressing Transformations and Poisson Group Actions, Publ. RIMS, Kyoto Univ. 21 (1985) 1237-1260

[71] M. A. Semenov-Tian-Shansky, Poisson-Lie Groups, Quantum Duality Principle and the Twisted Quantum Double, Theor. Math. Phys. 93, nr. 2 (1992) $302-329$ 
[72] A. Sen, Strong-Weak Coupling Duality in Four Dimensional String Theory, Int. J. Mod. Phys. A9 (1994) 3707-3750; hep-th/9402002

[73] A. Sen, Do Branes on $T^{n}$ and Matrix Theory, Adv. Theor. Math. Phys. 2 (1998) 51-59; hep-th/9709220

[74] A. Sen, An Introduction to Non-perturbative String Theory, hep-th/9802051

[75] A. Strominger, Massless Black Holes and Conifolds in String Theory, Nucl. Phys. B451 (1995) 96-108; hep-th/9504090

[76] L. Susskind, Another Conjecture about M(atrix) Theory, hep-th/9704080

[77] P. K. Townsend, The eleven-dimensional supermembrane revisited, Phys. Lett. B350 (1995) 184-187

[78] W. Taylor IV, D-brane Field Theory on Compact Spaces, Phys. Lett. B394 (1997) 283 ; hep-th $/ 9611042$

[79] W. Taylor IV, Lectures on D-branes, Gauge Theory and M(atrices), hepth/9801182

[80] G. 't Hooft, A Property of Electric and Magnetic Flux in Nonabelian Gauge Theories, Nucl. Phys. B153 (1979) 141

[81] G. 't Hooft, Some Twisted Self-Dual Solutions for the Yang-Mills Equations on a Hypertorus, Commun. Math. Phys. 81 (1981) 267-275 
[82] A. Tsuchiya, Y. Kanie Vertex Operators in the Conformal Field Theory on $\mathrm{P}^{1}$ and Monodromy Representations of the Braid Group, Letters in Math. Phys. 13 (1987) 303-312

[83] B. de Wit, J. Hoppe, H. Nicolai On the Quantum Mechanics of Supermembranes Nucl. Phys. B (1988) 305-545

[84] E. Witten, D. Olive, Supersymmetry Algebras that include Topological Charges Phys. Lett. 78B (1978) 97

[85] E. Witten, Constraints on Supersymmetry Breaking, Nucl. Phys. B202 (1982) 253

[86] E. Witten, String Theory Dynamics in Various Dimensions, Nucl. Phys. B443 (1995) 85-126; hep-th/9503124

[87] E. Witten, Bound States of Strings and p-Branes, Nucl. Phys. B460 (1996) 335-350; hep-th/9510135

[88] B. Zumino, Introduction to the Differential Geometry of Quantum Groups, K.Schmüdgen (Ed.), Math. Phys. X, Proc. X-th IAMP Conf. Leipzig (1990), Springer-Verlag (1990)

[89] B. Zumino, Differential Calculus on Quantum Spaces and Quantum Groups, XIX ICGTMP, M. O., M. S. and J. M. G. (Ed.), CIEMAT/RSEF, Madrid, vol. 1 (1993) 4 